LBNL-44249

\title{
DC SQUID Spectrometers for Nuclear Quadrupole and Low-Field Nuclear Magnetic Resonance Spectroscopy
}

\author{
Dinh M. TonThat \\ Ph.D. Thesis \\ Department of Physics \\ University of California, Berkeley \\ and \\ Materials Sciences Division \\ Ernest Orlando Lawrence Berkeley National Laboratory \\ University of California \\ Berkeley, CA 94720
}

April 1998

This work was supported by the Director, Office of Science, Office of Basic Energy Sciences, Materials Sciences Division, of the U.S. Department of Energy under Contract No. DE-AC03-76SF00098. 


\section{DISCLAIMER}

This report was prepared as an account of work sponsored by an agency of the United States Government. Neither the United States Government nor any agency thereof, nor any of their employees, make any warranty, express or implied, or assumes any legal liability or responsibility for the accuracy, completeness, or usefulness of any information, apparatus, product, or process disclosed, or represents that its use would not infringe privately owned rights. Reference herein to any specific commercial product, process, or service by trade name, trademark, manufacturer, or otherwise does not necessarily constitute or imply its endorsement, recommendation, or favoring by the United States Government or any agency thereof. The views and opinions of authors expressed herein do not necessarily state or reflect those of the United States Government or any agency thereof. 


\section{DISCLAIMER}

Portions of this document may be illegible in electronic image products. Images are produced from the best available original document. 


\begin{abstract}
DC SQUID Spectrometers for Nuclear Electric Quadrupole and Low Field

Nuclear Magnetic Resonance Spectroscopy

by
\end{abstract}

Dinh Minh That Ton

Doctor of Philosophy in Physics

University of California at Berkeley

Professor John Clarke, Chair

The dc Superconducting Quantum Interference Device (SQUID) is a very sensitive detector of magnetic flux, with a typical flux noise of the order of $1 \mu \Phi_{0} \mathrm{~Hz}^{-1 / 2}$ at liquid helium temperature $\left(\Phi_{0}=h / 2 \mathrm{e}\right)$. This inherent flux sensitivity of the SQUID is used in a spectrometer for the detection of nuclear magnetic resonance (NMR) and nuclear quadrupole resonance (NQR). The precessing magnetic field from the nuclear spins is coupled to the SQUID by mean of a flux transformer. An NMR probe configuration consists of a static field coil, an audio frequency (af) pulsing coil, and a pickup coil coupled to the SQUID.

The SQUID NMR spectrometer is used to measure the longitudinal relaxation time $T_{1}$ of solid ${ }^{129} \mathrm{Xe}$ at $4.2 \mathrm{~K}$ down to $0.1 \mathrm{mT}$. The long relaxation time of $\sim 2000$ seconds at these low fields is of key importance for the long term storage of hyperpolarized ${ }^{129} \mathrm{Xe}$, which can be achieved through optical pumping of rubidium and subsequent spin- 
exchange. Xenon can be polarized up to $10^{5}$ times over the thermally equilibrium value by this means. The possibility of using highly polarized gases such as ${ }^{129} \mathrm{Xe}$ and ${ }^{3} \mathrm{He}$ for low field magnetic resonance imaging (MRI) was also investigated. One dimensional images of ${ }^{3} \mathrm{He}$ gases and two dimensional images of solid ${ }^{129} \mathrm{Xe}$ were taken at static fields down to $0.5 \mathrm{mT}$, with resolutions of $500 \mu \mathrm{m}$ and $950 \mu \mathrm{m}$, respectively. However, higher resolution images can be achieved.

A modified SQUID spectrometer is used to detect NQR in zero field. The NQR configuration consists of a solenoid af pulsing coil and a coaxial pickup coil with a few turns. The NQR spectrometer is used to detect $\mathrm{NQR}$ spectra of ${ }^{27} \mathrm{Al}$ in $\mathrm{Al}_{2} \mathrm{O}_{3}\left[\mathrm{Cr}^{3+}\right]$ and ${ }^{2} \mathrm{D}$ in perdeuterated picoline and toluene. The ${ }^{2} \mathrm{D}$ picoline and toluene spectra have resonances near $40 \mathrm{kHz}$ possibly showing a quantum tunneling rotation within the $\mathrm{CD}_{3}$ group. The NQR lines of ${ }^{27} \mathrm{Al}$ occur at a higher frequencies, $359 \mathrm{kHz}$ and $714 \mathrm{kHz}$. Two-dimensional $\mathrm{NQR}$ of ${ }^{27} \mathrm{Al}$ was also demonstrated; this technique can be used to correlate resonances in more complicated spectra. The spectrometers are designed to cover frequencies up to $5 \mathrm{MHz}$, opening up the possibility of detecting pure NQR in many compounds containing ${ }^{14} \mathrm{~N},{ }^{7} \mathrm{Be},{ }^{11} \mathrm{~B}$, and ${ }^{27} \mathrm{Al}$.

Experiments involving the Overhauser Effect were performed in a single-coil double-channel probe. The enhancement of the ${ }^{1} \mathrm{H}$ nuclear magnetization was 183 in a $\mathrm{MnCl}_{2} / \mathrm{H}_{2} \mathrm{O}$ solution. The SQUID spectrometer was redesigned for performing Overhauser Effect experiments. If these experiments are successful, the enhanced signal and 
reduced $T_{1}$ may revolutionize the field of SQUID-detected NMR. 


\section{Table of Contents}

\section{Table of Contents}

List of Figures

Acknowledgements

Chapter 1 Motivation

Chapter 2 Development of SQUID NMR and NQR Spectrometers

2.1 Introduction

2.2 SQUID Basics

7

2.3 SQUID and Flux Transformer

8

2.4 Additional Positive Feedback and Flux-Locked Loop

2.5 Spectrometer

\section{Chapter 3 NMR of Laser-Polarized Xenon at 4.2 K}

3.1 Introduction

3.2 NMR Theory

3.3 Optical Pumping and Spin Exchange

3.3.1 Optical Pumping

3.3.2 Spin Exchange

3.4 Experimental Configuration

3.5 Results and Discussion 
Chapter 4 SQUID MRI of Laser-Polarized ${ }^{3} \mathrm{He}$ and ${ }^{129} \mathrm{Xe}$ at $4.2 \mathrm{~K}$

$\begin{array}{lll}4.1 & \text { Introduction } & 49\end{array}$

4.2 MRI Theory 51

4.2.1 Basic Theory $\quad 52$

4.2.2 Two-Dimensional Fourier Imaging 54

4.2.3 Projection Reconstruction 56

4.3 Experimental Setup $\quad 57$

4.4 Results and Discussion $\quad 59$

4.4.1 One-Dimensional MRI of ${ }^{3} \mathrm{He} \quad 59$

4.4.2 Two-Dimensional MRI of Solid ${ }^{129} \mathrm{Xe} \quad 62$

$\begin{array}{lll}4.5 & \text { Future Work } & 64\end{array}$

Chapter $5 \quad$ SQUID-Detected NQR of ${ }^{27} \mathrm{AI}$ and ${ }^{2} \mathrm{D}$

$\begin{array}{lll}5.1 & \text { Introduction } & 67\end{array}$

$\begin{array}{lll}5.2 N Q R \text { Theory } & 70\end{array}$

$\begin{array}{lll}5.2 .1 \eta=0 & 71\end{array}$

$\begin{array}{ll}5.2 .2 \eta \neq 0 & 72\end{array}$

$\begin{array}{lll}5.3 & \text { Experimental Configuration } & 75\end{array}$

$\begin{array}{lll}5.4 & \text { Result } & 77\end{array}$

5.4.1 ${ }^{27} \mathrm{Al}$ in Ruby (One-Dimensional)

5.4.2 Two-dimensional NQR $-{ }^{27} \mathrm{Al}$ of Ruby $\quad 81$ 
Chapter 6 Dynamic Nuclear Polarization at Low fields

$\begin{array}{ll}6.1 & \text { Introduction }\end{array}$

$\begin{array}{lll}6.2 & \text { Theory } & 94\end{array}$

6.3 Experimental Configuration 98

$\begin{array}{lll}\text { 6.3.1 Probe } & 98\end{array}$

6.3.2 Spectrometer and Duplexer 99

$\begin{array}{lll}6.4 & \text { Result and Discussion } & 101\end{array}$

$\begin{array}{ll}\text { 6.4.1 Performance } & 101\end{array}$

$\begin{array}{ll}\text { 6.4.2 Pulsed DNP } & 102\end{array}$

$\begin{array}{lll}\text { 6.5 SQUID DNP Experiment } & 106\end{array}$

$\begin{array}{lll}\text { Appendix A SQUWD Electronics } & 109\end{array}$ 


\section{List of Figures}

$\begin{array}{lll}2.1 & \text { SQUID basic operation } & 7\end{array}$

$\begin{array}{lll}2.2 & \text { Picture of a dc SQUID } & 9\end{array}$

$\begin{array}{lll}2.3 & \text { SQUD package } & 11\end{array}$

$\begin{array}{lll}2.4 & \text { Flux transformer } & 12\end{array}$

$\begin{array}{lll}2.5 & \text { APF Circuit and V-F curves } & 14\end{array}$

$\begin{array}{lll}2.6 & \text { Flux-locked Loop } & 16\end{array}$

$\begin{array}{lll}2.7 & \text { Gain vs. frequency } & 17\end{array}$

$\begin{array}{lll}2.8 & \text { Flux noise vs. frequency } & 19\end{array}$

$2.9 \quad$ Block diagram of NQR spectrometer 20

$\begin{array}{lll}2.10 & \text { JFET blanking switch . }\end{array}$

3.1 Energy level diagram for spin $\mathrm{I}=1 / 2$

3.2 Magnetization in the presence of a rf field 27

$\begin{array}{lll}3.3 & \text { Optical pumping } & 31\end{array}$

$\begin{array}{lll}3.4 & \text { Spin exchange processes } & 32\end{array}$

$\begin{array}{lll}3.5 & \text { Optical pumping setup } & 34\end{array}$

$\begin{array}{lll}3.6 & \text { NMR Cell } & 36\end{array}$

3.7 Free induction decay of optically pumped solid ${ }^{129} \mathrm{Xe}$.

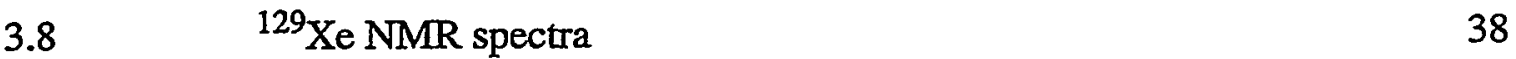

$\begin{array}{ll}{ }^{129} \mathrm{Xe} \text { linewidth vs. magnetic field } & 39\end{array}$

$\begin{array}{lll}3.10 & \text { Normalized magnetization vs. times }\end{array}$ 
3.11 T1 relaxation rate vs. magnetic field

3.12 Sample tube in the cross polarization experiments

4.1 Spectra in the presence of an homogeneous static magnetic field

4.2 Spectra in the presence of a gradient field

4.3 Signal in an MRI experiment

4.4 Pulse sequence in a 2-D Fourier Imaging experiment

4.8 Spin echo of ${ }^{3} \mathrm{He}$ signal $\quad 60$

4.9 1-D MRI of ${ }^{3} \mathrm{He}$ gas at different gradients

4.10 MRI of ${ }^{129} \mathrm{Xe}$ solid

5.3 Energy level diagram for $I=1$

5.4 Energy level diagram for $I=3 / 2$ 
5.11 Pulse sequence in a 2-D NQR experiment

5.14 High field ${ }^{2} \mathrm{D} N \mathrm{NMR}$ spectra of the $\mathrm{CD}_{3}$ group

5.15 Pure NQR spectra of perdeuterated toluene and picoline

6.1 The energy level diagram for the electron-nucleus system 94

6.2 Energy level diagram with relaxation rates 95

6.3 Enhancement of the nuclear magnetization vs. pumping rate 97

6.4 Two-channel probe for low field Overhauser Effect experiment 98

6.5 Spectrometer for the two-channel probe 100

$\begin{array}{lll}6.6 & \text { Pulse sequence } & 103\end{array}$

6.7 ${ }^{1} \mathrm{H}$ spectra at different saturation time of the electron channel $\quad 104$

6.8 $\quad{ }^{1} \mathrm{H}$ spectra at different pumping rate of the electron channel 104

$\begin{array}{lll}6.9 & \text { Comparison of the }{ }^{1} \mathrm{H} \text { spectra } & 105\end{array}$

$\begin{array}{lll}6.10 & \text { SQUID DNP setup } & 106\end{array}$ 


\section{Acknowledgments}

There is a Vietnamese saying "when you eat the fruits, remember the ones who planted and cared for the trees."

With that, I first want to thank Professor John Clarke for allowing me the opportunity to work in his lab, for giving me many guidances during my research, and for providing me with the necessary tools and a good environment to achieve my goals. I am greatly indebted to him and proud that he is my research advisor. For the second half of my research, I spent most of my time in Professor Alex Pines' lab. I thank him for providing a wonderful place to work and the many talented post-docs and gradudate students during our collaboration.

The project was in its infancy when I first joined and I bad to rely on many individuals for their help and guidance. In my days of fabricating SQUIDs, I appreciate Jack Kingston, Jamie Yarvis, and Nancy Missert for their suggestions and valuable lessons. The SQUID electronics in Chapter 2 would not be possible without John Davis in the Electronic Shop of the Physics Department and Martin Hurlimann. I have learned many things from John Davis and I thank him for spending time with me.

In collaboration with Professor Alex Pines in the Chemistry Department, I especially wish to thank Marcia Ziegeweid for my helping me to adjust to Professor Pines Group and for collaborating with me on the xenon project. I also thank her for the many stories and all the gossip in the Pines Group she told. I am very happy to have a chance to work with Matthew Augustine. I benefitted a lot from his efforts and dedication during our 
collaborations. I enjoyed learning and conversing with many graduate students and postdocs during my years at Cal, including (but not nearly limited to) Tom Lee, Gene Dantsker, Andy Miklich, Tim Shaw, Theresa Ho, Beth Chen, Konstantin Kouzetsnov, Annjoe Wongfoy, Marco Tomaselli, Jeff Yarger, Yi-Qiao Song, David DeGraw, Seth Bush, Boyd Goodson, Dave Laws, Tanja Pietrass, many other members of Professor John Clarke's group and Pinenuts. It was fun to be a part of such a big family.

I also want to acknowledge Dione Carmichael and Barbara Salisbury. I am sure I caused them a few headaches. I appreciate your cares. I thank the Mircrolab for the use of their facility to fabricate SQUIDs. This work was supported by the Director, Office of Energy Research, Materials Sciences Dvision of the U.S. Department of Energy under Contract No. DE-AC03-76SF00098.

Finally, this thesis is dedicated to my mother for her love. My undying gratitude to my siblings in the US for keeping me sane and cheerful during the holidays from my research days at $\mathrm{Cal}$. 


\section{Chapter 1}

\section{Motivation}

High-Field Nuclear Magnetic Resonance (NMR) is an immensely powerful tool to study many processes at the atomic level. The nuclear magnetic dipole-dipole interaction or electric quadrupole interactions obtained, in turn, yield valuable information on interatomic distances and local electric field gradients [1-3]. In polycrystalline or disordered materials, however, the molecules are randomly oriented with respect to the external applied magnetic field, and the resulting "powder spectra" may well conceal any structural information, especially if the interactions are relatively weak. One solution to this problem is to remove the external magnetic field, which defines a spatial direction, and to measure the Nuclear Quadrupole Resonance (NQR) [4] or zero-field NMR [5] directly; since all equivalent sites have the same resonant frequency the resulting spectra should be sharp. Examples of $\mathrm{NQR}$ are ${ }^{9} \mathrm{Be},{ }^{11} \mathrm{~B},{ }^{14} \mathrm{~N}$, and ${ }^{27} \mathrm{Al}$ and ${ }^{2} \mathrm{D}$ in selectively deuterated organic molecules; an example of zero-field NMR is the proton-proton dipole resonance in polycrystalline materials. 
Although working in a low field or a zero field environment is an attractive approach to the acquisition of structural information of noncrystalline materials, the disadvantage is that often the signals acquired are very small due to the small resonant frequency of the sample. Typical zero field NQR line of ${ }^{14} \mathrm{~N}$ in the ammonium ion is a few tens of kilohertz, ${ }^{14} \mathrm{~N}$ in a $\mathrm{NH}_{3}$ is a few hundreds of kilohertz. As a result, the voltage signal developed across a Faraday coil surrounding a collection of precessing spins in a normal NMR setup is greatly reduced compared to that at hundreds of megahertz. At a temperature $T$, the magnetization $M$ scales as $\omega / T$ and the induced voltage as $\omega M$, so that the signal scales as $\omega^{2} / T$. As one goes from detecting a signal at $100 \mathrm{MHz}$ to that of detecting a signal at $100 \mathrm{kHz}$, the reduction in signal by 6 orders of magnitude or more makes it difficult to observe low-frequency NMR or NQR with a conventional semiconductor amplifier coupled to a resonant circuit. Alternative methods have been developed. One method is magnetic field cycling [6,7], which produces the free induction decay (FID) point-by-point, and is thus rather time-consuming. The principle idea behind that is, first, the sample is allowed to polarize in a high field region and then allowed to evolve for some time $t$ in a region of zero field. Quickly the sample is transported back to the bore of the magnet where the magnitude of the evolved magnetization is detected. The FID is this magnitude versus the evolving time $t$. Another approach, involving a Robinson oscillator [8] in a continuous wave (cw) NQR spectrometer, has been successfully at frequencies down to $200 \mathrm{kHz}[9]$.

In order for the most powerful experiments to become routine, a method need to be developed for direct observation of the precessing magnetization in zero field. The method should be capable of high sensitivity and of detecting signal down to a few hertz resonant. 
The superconducting quantum interference device (SQUID) is the world most flux sensitive detector, and at kilohertz frequencies, the SQUID can have a noise temperature far below that of conventional amplifiers [10]. The unique feature of the SQUID is that it can directly detect flux, rather than the rate change of magnetic flux, so that at a given temperature the detected signal scales as $\omega$ rather than as $\omega^{2}$. The combination of direct flux detecting and the high flux sensitivity make the SQUD an alternative approach to the conventional Faraday detector for the detection of NMR and NQR signal down to the hertz frequencies.

In Chapter 2, I will discuss this SQUID spectrometer development. Chapters 3-6, I will report on the many different types of experiment done with the SQUID spectrometer: to detect NMR and MRI of optically pumped ${ }^{129} \mathrm{Xe}$ and ${ }^{3} \mathrm{He},{ }^{2} \mathrm{D}$ NQR spectra of picoline and toluene, ${ }^{27} \mathrm{Al} \mathrm{NQR}$ spectra of ruby.

Finally, I will conclude with a current research project involving detection of NMR and NQR signal with Overhauser Effect using the SQUID spectrometer. 


\section{References}

[1] M. Mehring, Principles of High Resolution NMR in Solids, (Springer-Verlag, Berlin, 1983.)

[2] C.P. Slichter, Priciples of Magnetic Resonance, (Springer-Verlag, Berlin, 1983.)

[3] A. Pines, in: W.A. Little (Ed.), Proceedings of the Bloch Symposium, (World Scientific, Singapore, 1990), pg 1241.

[4] T.P. Das and E.L. Hahn, Solid State Supplement 1 : Nuclear Quadrupole Resonance Spectroscopy, (Acadamic Press, New York, 1958.)

[5] N.F. Ramsey, R.V. Pound, Phys. Rev. 81 (1951) 278.

[6] D.T. Edmonds, Phys. Rep. 29 (1977) 233.

[7] F. Noack, Progr. NMR Spectrosc. 18 (1986) 171.

[8] F.N.H. Robinson, J. Phys. E: Sci. Instrum. 15 (1982) 814.

[9] D. Lee, S.J. Gravina, P.J. Bray, Z. Naturforsch. 45A (1990) 268.

[10] J. Clarke, in: H. Weinstock, R. Ralston, SQUIDS: Theory and Practice, The New Superconducing Electronics, (Kluwer Academic, Dordrecht, 1993), p.123. 


\section{Chapter 2}

\section{Development of a SQUID NMR and NQR Spectrometer}

\subsection{Introduction}

Earlier, Non Q. Fan, a former student of my advisor John Clarke, successfully built and operated a SQUID NMR and NQR spectrometer to detect ${ }^{14} \mathrm{~N} \mathrm{NQR}$ of $\mathrm{NH}_{4} \mathrm{ClO}_{4}$, and NMR of Pt and Cu[1]. The SQUID spectrometer was limited to a frequency range of about $200 \mathrm{kHz}$. However, there are many nuclei of considerable interest - for example, ${ }^{9} \mathrm{Be},{ }^{11} \mathrm{~B},{ }^{14} \mathrm{~N}$, and ${ }^{27} \mathrm{Al}$ - that have resonant frequencies up to several megahertz. In this chapter, I will describe the development of a SQUID NMR and NQR spectrometer operating at frequencies up to $5 \mathrm{MHz}$. This development will enable NMR researchers to perform many different low-frequency pulsed-NMR experiments which have presented difficulties for the current state of the art of high field solid state NMR techniques, such as the direct detection of ${ }^{14} \mathrm{~N} N Q R$ in organic molecules and proteins, or ${ }^{11} \mathrm{~B}$ and ${ }^{27} \mathrm{Al} \mathrm{NQR}$ of ceramic materials and nanotubes. 
In the previous SQUID NMR and NQR spectrometer, applications were limited to resonant frequencies below $200 \mathrm{kHz}$ by the SQUID electronics. The reason for this limitation is that typical dc SQUID electronics makes use of a modulation frequency to be mixed with the SQUID signal in order to optimize the SQUID system noise performance in the low frequency regime. In this modulation and lock-in detection scheme, the low frequency response of the SQUID electronics is not limited by the preamplifier but by the SQUID intrinsic $1 / \mathrm{f}$ noise. The modulation frequency was $500 \mathrm{kHz}$, and thus the maximum operational bandwidth of the spectrometer was less than half of this modulation frequency or about $200 \mathrm{kHz}$. One question to ask is why this modulation frequency cannot be made higher, say like $10 \mathrm{MHz}$ or so. At such high frequency, the noise of the preamplifier is not only much higher, but the gain-bandwidth product (GBW) of typical commercial amplifiers is not large enough to accommodate the high gain required by the SQUWD electronics.

The new SQUID electronics is operated in a flux-locked loop using a modified version of the direct offset integration technique (DOIT) with additional positive feedback (APF) developed by Drung et. al. [2-4], in which the output of the SQUID is directly coupled to a low noise amplifier operated at room temperature. Since the SQUDD is not flux modulated, the frequency response of the system is determined entirely by the design of the flux locked loop. This design lacks the low frequency sensitivity of the modulated flux-locked loop scheme; however, since we are working at frequencies greater than a few tens of hertz, this noise problem is irrelevant. This circuit has been used by Thomasson and Gould [5] in a circuit configured for NMR measurements at frequencies up to about $300 \mathrm{kHz}$ and by me to frequencies up to $5 \mathrm{MHz}[6]$. I will briefly describe the operation of 
a dc SQUID and then go into detail on the different aspects of the new SQUID NMR and NQR spectrometer.

\subsection{SQUID Basics}

The principle operation of a dc SQUID is extensively discussed elsewhere [7].
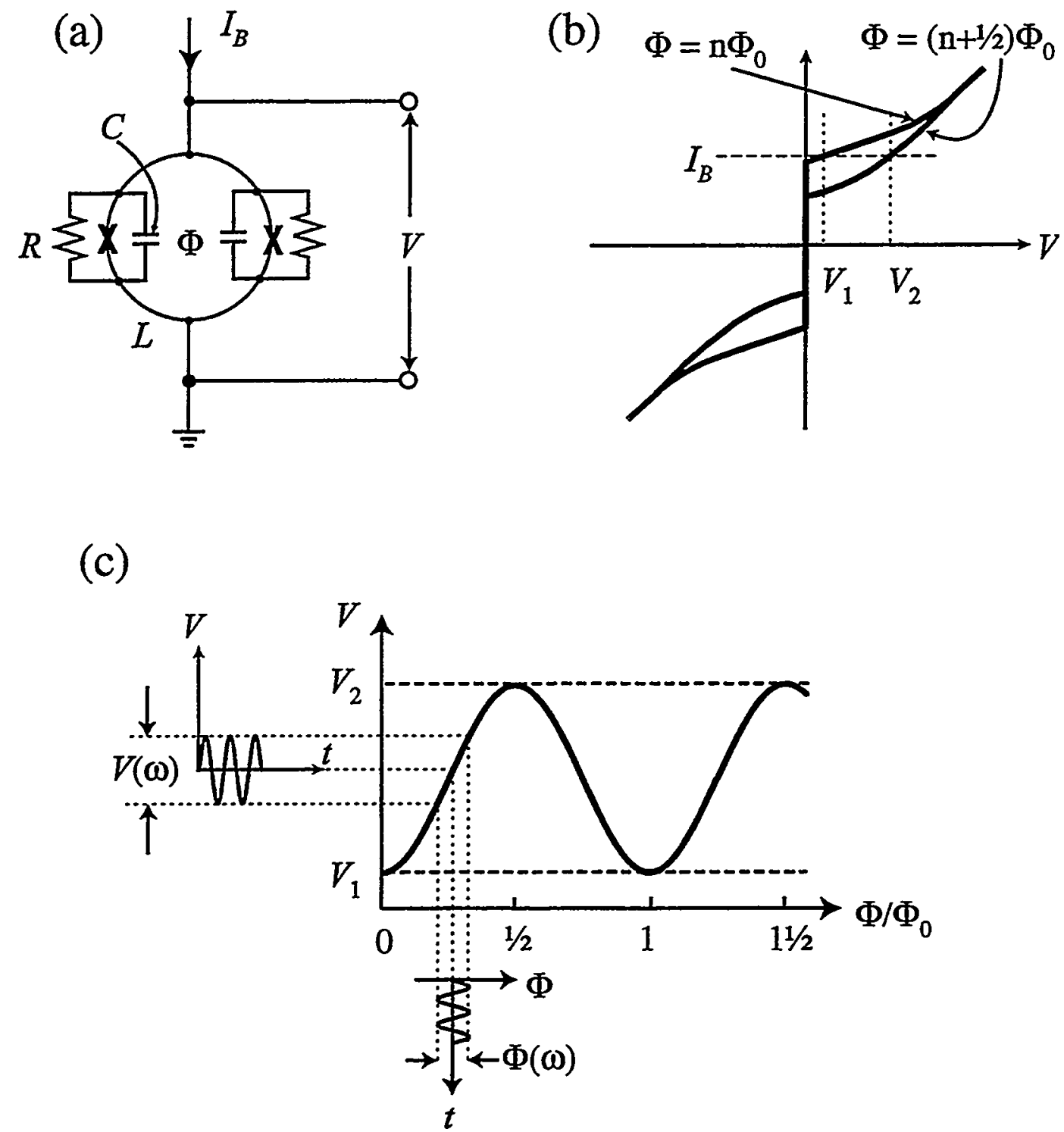

Figure 2.1: (a) Schematics of a dc SQUID; (b) current-voltage (I-V) characteristics for an applied flux $\Phi$ of $n \Phi_{0}$ or $(n+1 / 2) \Phi_{0}$; (c) voltage-flux $(V-\Phi)$ curve for constant bias current $I_{B}$, indicating how a small flux $\Phi(\omega)$ is converted into voltage $\mathrm{V}(\omega)$. 
However, I will, for completeness, summarize its essential points here. A dc SQUID consisted of a superconducting loop of inductance $L$ interrupted by two Josephson tunnel junctions [8] of self-capacitance $C$ as shown in Figure 2.1(a). The Josephson junction is damped by a resistance $R$ to produce the nonhysteretic current-voltage $(I-V)$ characteristic shown in Figure 2.1 (b). When one changes the magnetic flux applied to the SQUID loop, the critical current oscillates between a maximum value at $\Phi=n \Phi_{0}$ and a minimum value at $\Phi=(n+1 / 2) \Phi_{0}$; thus, the period of oscillation is $\Phi_{0}$, where $\Phi_{0}$ is a flux quantum $\left(\Phi_{0}=\hbar / 2 \mathrm{e}\right)$. For our operation, the SQUID is current-biased at a point $I_{B}$ above the maximum critical current where the modulation depth $\left(V_{1}-V_{2}\right)$ is a maximum value. In the current-biased mode, the SQUID voltage $V$ changes as a function of flux $\Phi$ as shown in Figure 2.1 (c). The SQUID is locked on the steepest region of the $V-\Phi$ curve to optimize its sensitivity. In effect, the SQUID is a flux to voltage transducer with a transfer coefficient $V_{\Phi}=(\partial \mathrm{V} / \partial \Phi)_{I}$. Often, the detected flux $\Phi(\omega)$ is much smaller than $\Phi_{0}$; the resulting SQUID output is $V(\omega)=\Phi(\omega) V_{\Phi}$

\subsection{SQUID and Flux Transformer}

The direct connection of the SQUID to the room temperature preamplifier somewhat restricts the kind of SQUID needed for the new spectrometer. Current commercial ultra-low noise amplifiers have a typical voltage of $1 \mathrm{nVHz}^{-1 / 2}$; since the flux noise of a typical SQUID is about 1 to $2 \mu \Phi_{0} \mathrm{~Hz}^{-1 / 2}$, a transfer function $V_{\Phi}$ of at least $500 \mu \mathrm{V} / \Phi_{0}$ to $1000 \mu \mathrm{V} / \Phi_{0}$ is needed to ensure that the dominant noise is not due to the room temperature amplifier. Computer simulations [9] for an optimized SQUID yield a maximum value 
of $V_{\Phi, \max } \approx R /$; our design values of $R=3 \Omega$ and $L=100 \mathrm{pH}$ yield $V_{\Phi, \max } \approx 60 \mu \mathrm{V} / \Phi_{0}$. This value is still way below that needed for an adequate SQUID transfer function. Unfortunately, we can not change the SQUID parameters to make $V_{\Phi}$ larger because making $R$ larger could result in a hysteretic I-V characteristics, and making $L$ smaller could result in a SQUID that is less efficient in magnetic flux coupling. However, as will be shown later on, the APF circuit proposed by Drung et. al. increases this transfer function by a factor of 10 and thus enables us to bypass this crucial problem.

A picture of the dc SQUID is shown in Figure 2.2. Modern SQUIDs are made

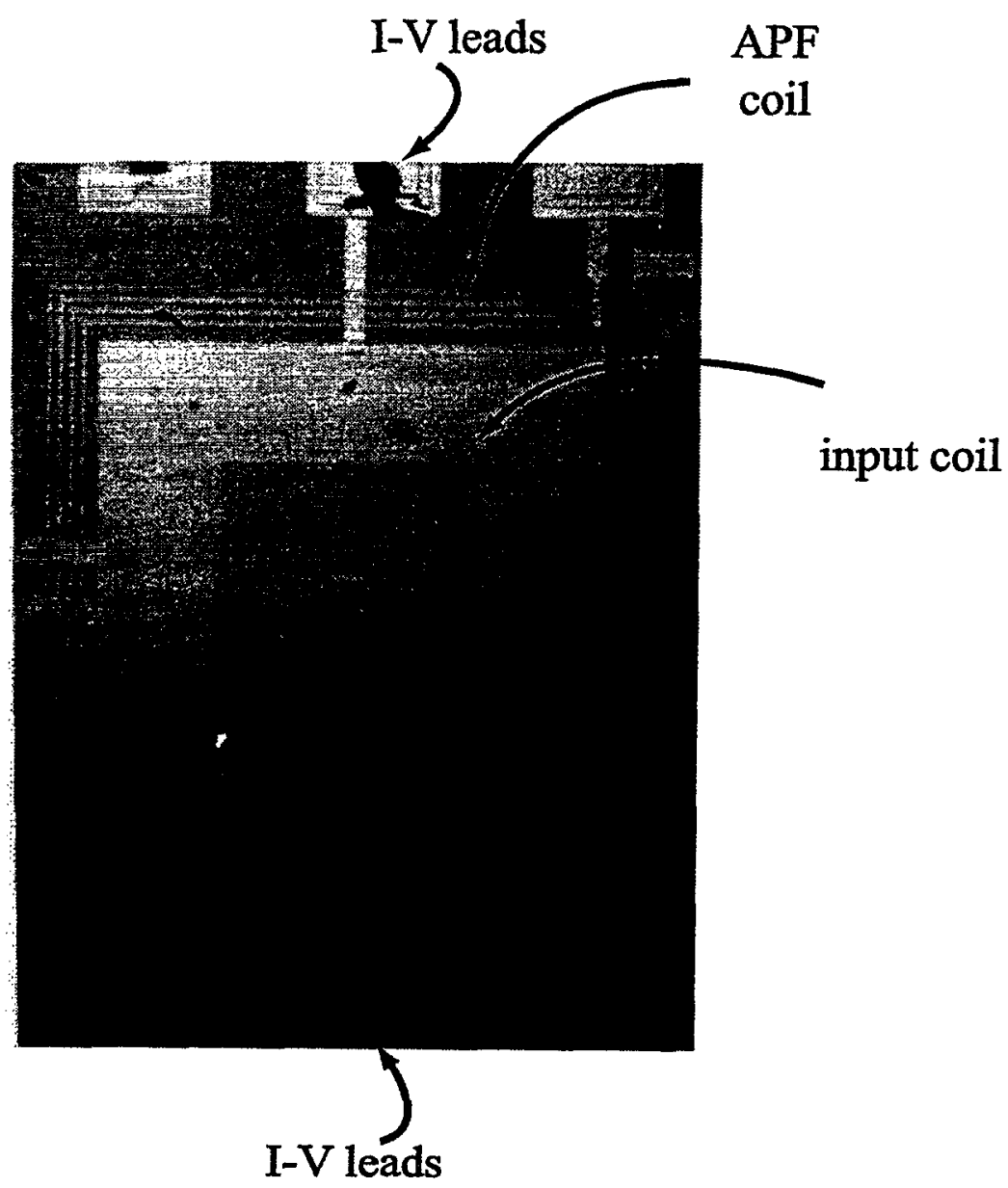

Figure 2.2: $\quad$ A picture of a dc SQUID. 
from thin films deposited on silicon wafers and patterned photolithographically. The SQUIDs are fabricated in batches of 144 on a $50 \mathrm{~mm}$-diam oxidized silicon wafer. SQUIDs designed in our laboratory have a $\mathrm{Pb} / \mathrm{In}$ counter electrode; details of the fabrication process can be found in Fred Wellstood's thesis [10]. Each SQUID consists of a Nb square washer[11] with inner and outer dimensions of 25 and $900 \mu \mathrm{m}$ on which are fabricated two $\mathrm{Nb}-\mathrm{NbO}_{\mathrm{x}}-\mathrm{Pb}(5 \% \mathrm{In})$ Josephson junctions. The process begins with a clean (100) Si wafer of $1 \mu \mathrm{m}$ thick $\mathrm{SiO}_{2}$. The CuAu shunt is first patterned with lift-off. Then a damping $\mathrm{CuAu}$ resistor is added. This damping resistor is designed to remove possible hysteretic and resonant behavior of the SQUID $I-V$ characteristic, due to inductive or capacitant coupling of the SQUID to its input coil, by suppressing the circulating current flowing around the SQUID loop[12,13]. After that, a first Nb layer is sputtered, patterned, and etched to form the SQUID washer and a 4-turn feedback coil surrounding the SQUID. Next, two SiO insulation layers are deposited through a lift-off mask to define the two Josephson junctions, $2 \mu \mathrm{mx} 2 \mu \mathrm{m}$ in size. Then a second $\mathrm{Nb}$ layer is patterned to form the input coil, crossovers, pads, and leads, following a photoresist layer patterned to protect these junctions and a milling step to clean the crossunder from the first $\mathrm{Nb}$ layer. The wafer is then patterned for a $\mathrm{Pb} / \mathrm{In}$ lift-off step and diced to provide individual SQUID chips before the last $\mathrm{Pb} / \mathrm{In}$ evaporation step is completed. The last process involves a milling step to clean off the $\mathrm{Nb}$ layer, an oxidation step in an $\mathrm{Ar} / \mathrm{O} 2(90 \% / 10 \%)$ plasma discharge to form $\mathrm{NbO}_{\mathrm{x}}$, and the final $\mathrm{Pb} / \mathrm{In}$ evaporation step to form the second superconducting layer of the junctions. Another SiO layer can also be added to protect the $\mathrm{Pb} / \mathrm{In}$ layer, which deteriorates with time. 
The SQUID inductance, measured from the critical current modulation depth of a hysteretic SQUID, is approximately $100 \mathrm{pH}$, of which about $60 \mathrm{nH}$ arises from the washer and $40 \mathrm{pH}$ from the slit in the washer separating the two junctions. Each junction has a typical critical current of $20 \mu \mathrm{A}$ and is shunted with a $3 \Omega$ resistor. In practice, the SQUID is still nonhysteretic even when the critical current is as high as $40 \mu \mathrm{A}$. The damping resistance across the SQUID slit is about $6 \Omega$ The 48-turn Nb spiral input coil deposited on the square washer has a mutual inductance to the SQUID of $3.5 \mathrm{nH}$ and an estimated self-inductance of about $150 \mathrm{nH}$. I made the superconducting contact to the input coil by wire-bonding $50 \mu \mathrm{m}$-diameter, annealed $\mathrm{Nb}$ wire to the $\mathrm{Nb}$ contact pads of the input coil, as shown in Figure 2.3, after spot welding the wire to a $\mathrm{Nb}$ foil. The annealed $\mathrm{Nb}$ wire is

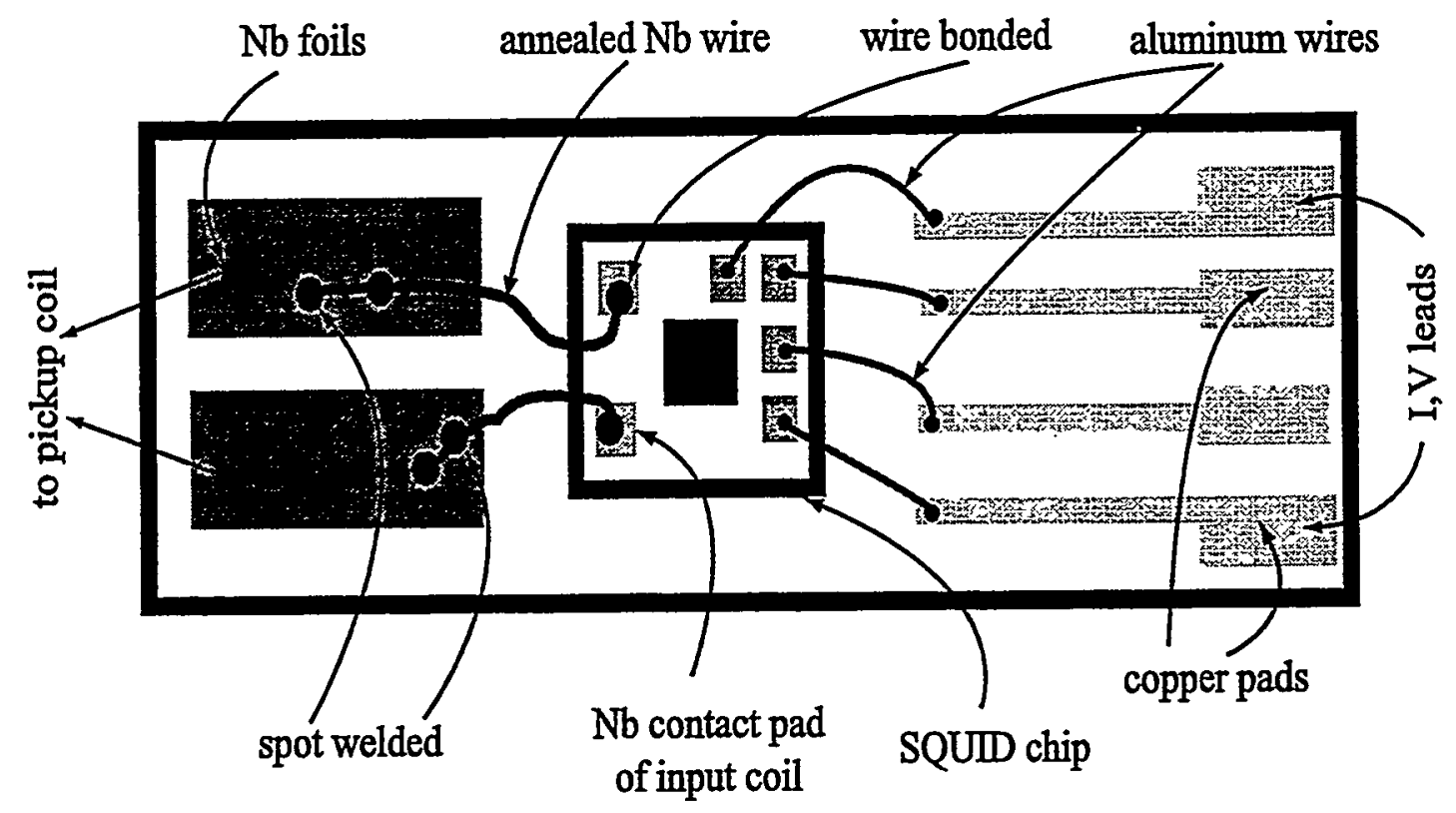

Figure 2.3: SQUID fiberglass holder. Annealed Nb wires connect the SQUID Nb contact pads of input coil to the $\mathrm{Nb}$ foils. Aluminum wires connect the SQUID current and voltage leads to the copper pads on top of the fiberglass piece. The whole assembly is then hermetically sealed leaving only the $\mathrm{Nb}$ foils and the copper pads exposed for connection. 
made by passing a $0.51-0.54 \mathrm{~A}$ current through a $15 \mathrm{~cm}$ length of $50 \mu \mathrm{m} \mathrm{Nb}$ wire in vacuum for 5 minutes. The SQUID current and voltage leads are wire bonded to the copper pads on top of the fiberglass SQUID holder. The SQUID is enclosed in a hermetically sealed G-10 fiberglass container that is surrounded by a $\mathrm{Nb}$ tube.

The sample is coupled to the input coil of the SQUID via a nonresonant superconducting circuit as shown in Fig 2.4, giving the spectrometer a broad bandwidth. The pickup coil is connected to the SQUID input coil by sandwiching the $\mathrm{Nb}$ pickup coil leads between $\mathrm{Nb}$ washers and the $\mathrm{Nb}$ foils to which the input coil is connected. A flux $\Phi(\omega)$ in the pickup loop induces a counter current $I(\omega)=-\Phi(\omega) /\left(L_{i}+L_{p}\right)$ in the flux transformer loop, where $L_{i}$ and $L_{p}$ are the input and pickup coil inductance. Passing through the input coil, this current produces a flux $-\Phi(\omega) M_{i} /\left(L_{i}+L_{p}\right)$ through the SQUID; $M_{i}$ is the mutual

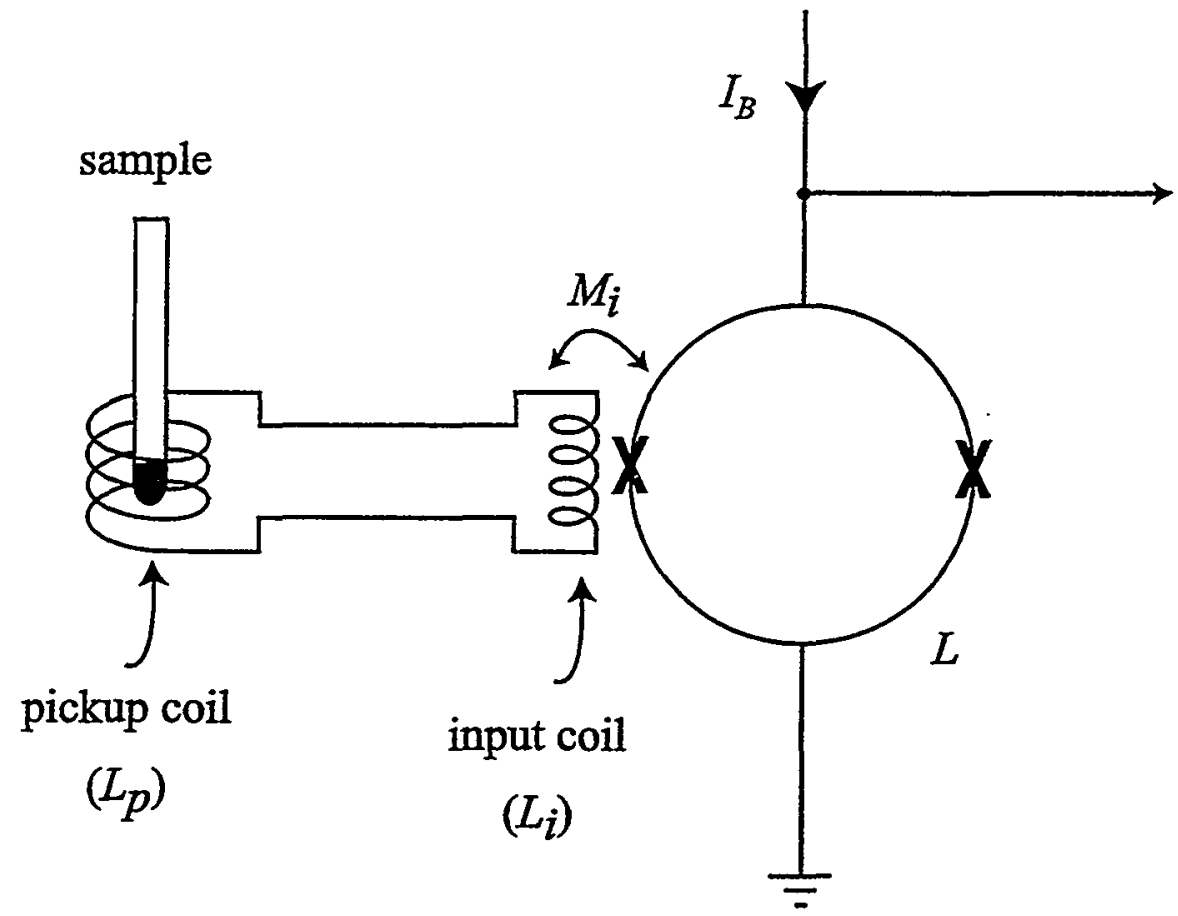

Figure 2.4: The flux transformer. The sample is placed in the pickup coil of inductance $L_{p}$. The flux from the sample induces a current which feeds flux into the SQUID loop as it passes through the input coil of inductance $L_{i}$. 
inductance between the SQUID and the input coil. To a first approximation, flux transfer from the pickup loop to the SQUID is optimized when $L_{i}=L_{p}$ and these results hold for frequencies ranging from zero up to the frequency at which parasitic capacitance becomes significant. For a 2-turn pickup coil of $1 \mathrm{~cm}$ diam, the inductance is about $100 \mathrm{nH}$.

\subsection{Additional Positive Feedback and Flux-Locked Loop}

The SQUID connected to the APF circuit is shown in Fig 2.5 (a). In essence, the SQUID is shunted by a coil of inductance $L_{\text {apf }}$, to which it is inductively coupled, in series with a cold MESFET (HMF 24020-100) that is used as a voltage-controlled resistor $\left(R_{\text {apf }}\right)$ in the range 2-25 $\Omega$ The coil is the 4-turn $\mathrm{Nb}$ wire microfabricated around the SQUID washer, and is patterned in the same layer; the mutual inductance $M_{\text {apf }}$ between the loop and the SQUID is about $380 \mathrm{pH}$. When a flux $\Phi$ is applied to the SQUID, the coil provides feedback that is positive or negative depending on the sign of the transfer function. The degree of the feedback is determined by the values of the mutual inductance, the feedback resistance, and the SQUID transfer function. As a result, the transfer function becomes asymmetric, with a steeper slope on the portion for which the feedback is positive as shown in Figure 2.5 (b). The transfer function is thus enhanced from a value of typically $50 \mu \mathrm{V} / \Phi_{0}$ up to values as high as $500 \mu \mathrm{V} / \Phi_{0}$. The new transfer function $V_{\Phi, \text { new }}$ can be quickly calculated as follow. The current flowing through the APF circuit is approximately $V I R_{a p f}$ feeding a flux $M_{a p f} V / R_{a p f}$ to the SQUID loop. The flux to voltage equation is then given by:

$$
\left(\Phi+\dot{M}_{a p f} V / R_{a p f}\right) V_{\Phi}=V .
$$


Solving for $V / \Phi$, we have

$$
V / \Phi=V_{\Phi, \text { new }}=V_{\Phi} /(1-\eta)
$$

where the feedback parameter $\eta=M_{a p f} V_{\Phi} / R_{a p f}$ The value of $V_{\Phi, \text { new }}$ is large when is $\eta$ approximately 1 and small compared to $V_{\Phi}$ when $\eta$ is negative; hence, its name "positive" feedback. To maintain stability, $|\eta|$ must be keep less than 1 for all possible values of $V_{\Phi}$.

(a)

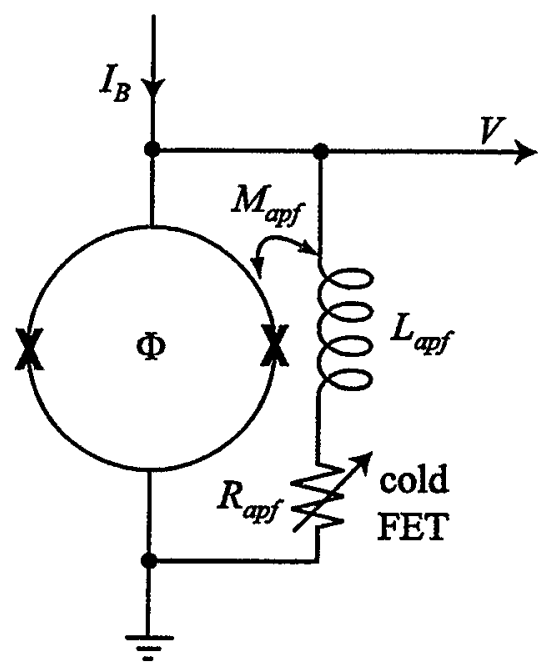

(b)

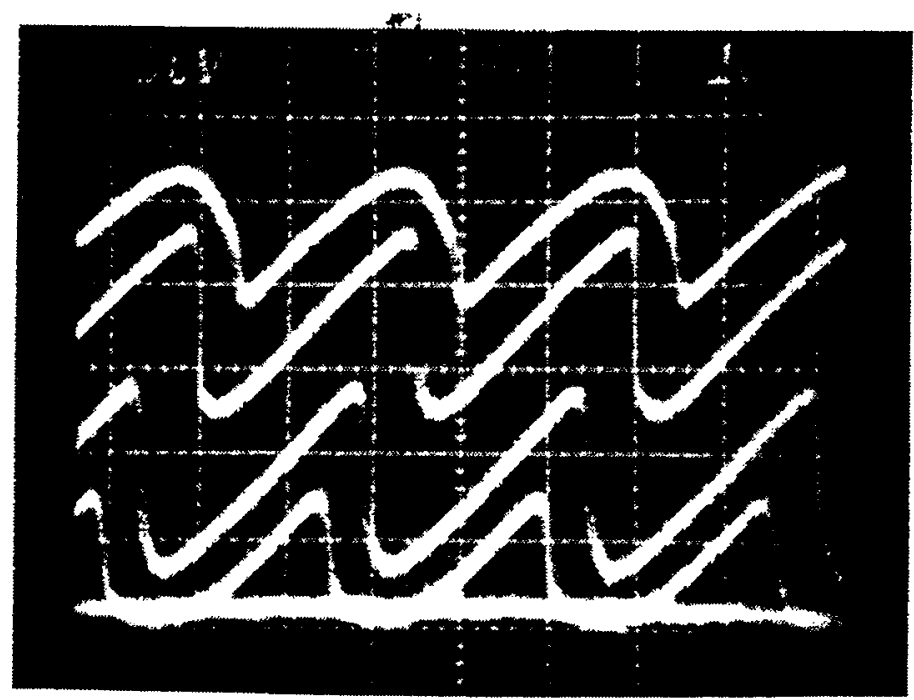

Figure 2.5: (a) APF scheme for the dc SQUID. (b) The V- $\Phi$ curves for different bias currents of the SQUID with APF circuit. 
It is important to think about the best values for $M_{a p f}$ and $R_{a p f}$ and the consequences of adding this circuit. To ensures that there is little phase shift in the APF circuit that can make the whole circuit unstable, $\omega_{3 d B} L_{a p f}$ must be much less than $R_{a p f}$, where $\omega_{3 d B}$ is the desired $3 d B$ cutoff frequency of the spectrometer. Thus, patterning the additional positive feedback coil directly on the SQUID chip rather than using a Nb-wire coil is good practice since this will keep the inductance low for the required value of $M_{a p f}$ Another important requirement is that the flux noise due to this additional positive feedback circuit be not much larger than the SQUID intrinsic flux noise $\Phi_{\mathrm{N}} \approx\left(16 \mathrm{k}_{\mathrm{B}} \mathrm{TR}\right)^{1 / 2} L$ [7]. The flux noise due to the APF circuit is the current noise in the feedback resistor passing through the feedback coil, $\left(4 \mathrm{k}_{\mathrm{B}} \mathrm{T} / R_{a p f}\right)^{1 / 2} M_{\text {apf }}$ For useful feedback $\eta \approx 1$ or $M_{\text {apf }} \approx R_{a p f} / V_{\Phi} \approx L R_{a p f} / R$; this gives $\left(16 \mathrm{k}_{\mathrm{B}} \mathrm{T} R\right)^{1 / 2} L\left(R_{a p f} / R\right)^{1 / 2} \leqslant 2 \Phi_{\mathrm{N}}$ or $R_{a p f} \leqslant 4 R$. Essentially, the feedback resistance must be less that 4 times the SQUID shunt resistance. Our typical $R_{a p f} \approx 10 \Omega$ ensures this condition.

A bias current $I_{\mathrm{B}}$ generates a voltage $V$ across the SQUID which is coupled directly to a low-noise preamplifier, as shown in Figure 2.6. Details of the flux locked loop electronic circuit can be found in Appendix A. The preamplifier consists of four AD797 (Analog Devices) amplifiers connected in parallel to reduce the voltage noise by a factor of two to a measured value of $0.65 \mathrm{nVHz}^{-1 / 2}$ at frequencies $(z 100 \mathrm{~Hz})$ above the $1 / f$ noise. The voltage gain is 6.5 . The current noise, about $4 \mathrm{pAHz}^{3 / 2}$, produces a negligible voltage noise across the SQUID, and the Nyquist noise of the leads connecting the SQUID 


\section{SQUID flux-locked loop}

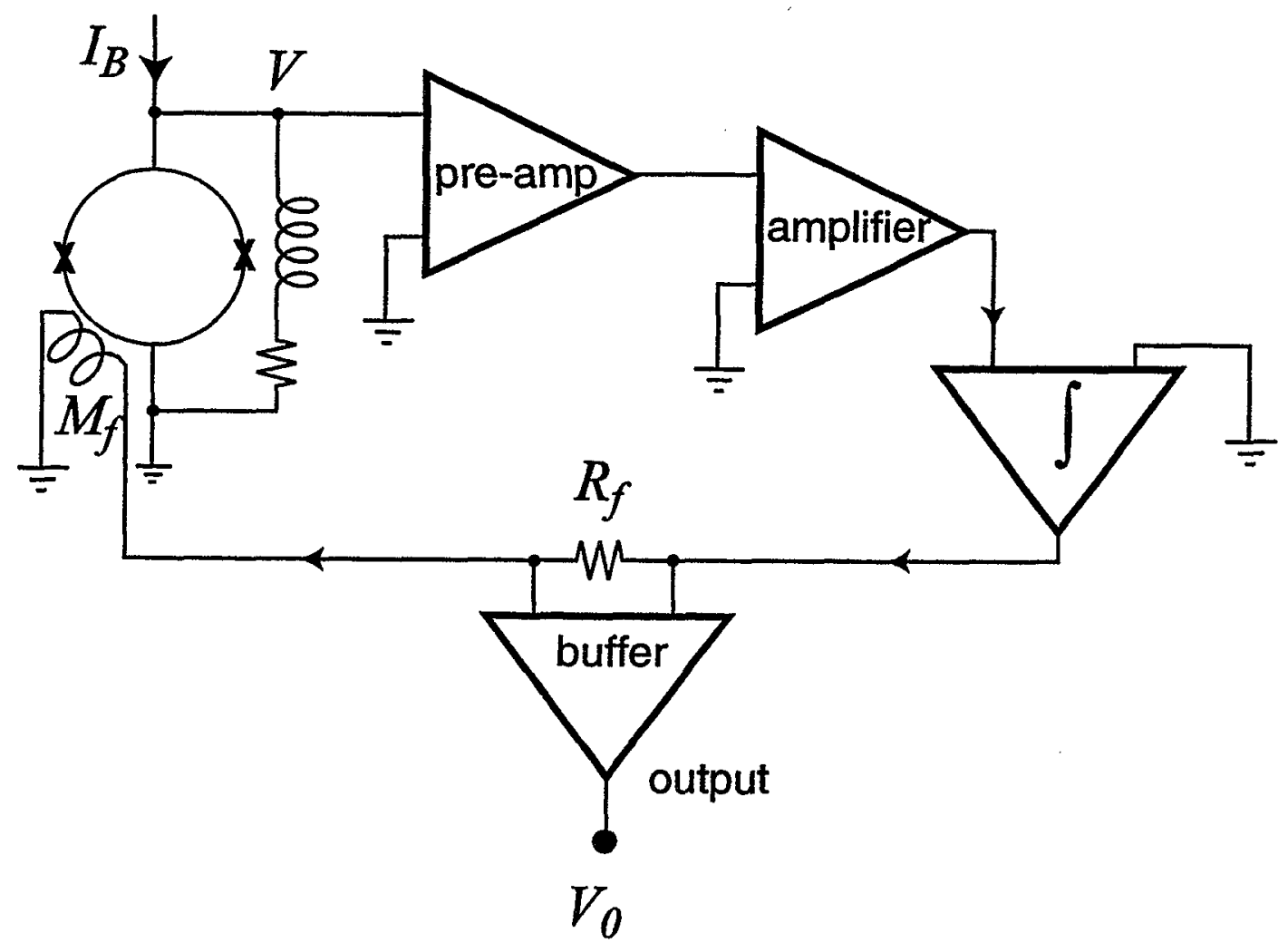

Figure 2.6: The SQUID flux locked loop. The output voltage is given by $R_{f} / M_{f}$ times the applied flux.

to the room temperature preamplifier is also insignificant. The preamplifier is followed by a second stage of amplification (Comlinear CLC 425 or AD797), variable from 15 to 40, and a single-pole integrator with an integration time constant $\tau_{i}$. The output from the integrator is coupled via the feedback resistor $R_{f}$ to the feedback coil that is coupled to the SQUID with a mutual inductance $M_{f}$ This coil, consisting of 20-30 turns of $\mathrm{Cu}$ wire, is glued into a small hole in the mount below the SQUID. This flux locked loop maintains the SQUID near the region of largest $V_{\Phi}$. The buffer stage provide noise isolation between 
the SQUID and the outside environment.

The output voltage $V_{0}$ from the flux-locked loop in response to an input flux $\Phi$ at the SQUID is

$$
V_{0}=\Phi\left(R_{f} / M_{f}\right) G(\omega) /[G(\omega)+1],
$$

where $G(\omega)=\left(R_{f} M_{f}\right) V_{\Phi} G_{a} G_{i}(\omega)$.

Here, $G_{a}$ is the voltage gain of the first two amplifier stages and $G_{i}(\omega)$ is the gain of the integrator, $1 / i \omega \tau_{i}$ at frequencies above about $1 \mathrm{kHz}$. For low frequencies $G(\omega)$ is very large; thus the output voltage is approximately proportionally to the flux in the SQUID. As $\omega$ increases, there will be a frequency $\omega_{3 d B}$ at which $\left|G\left(\omega_{3 d B}\right)\right|=1$; this frequency $\omega_{3 d B}$

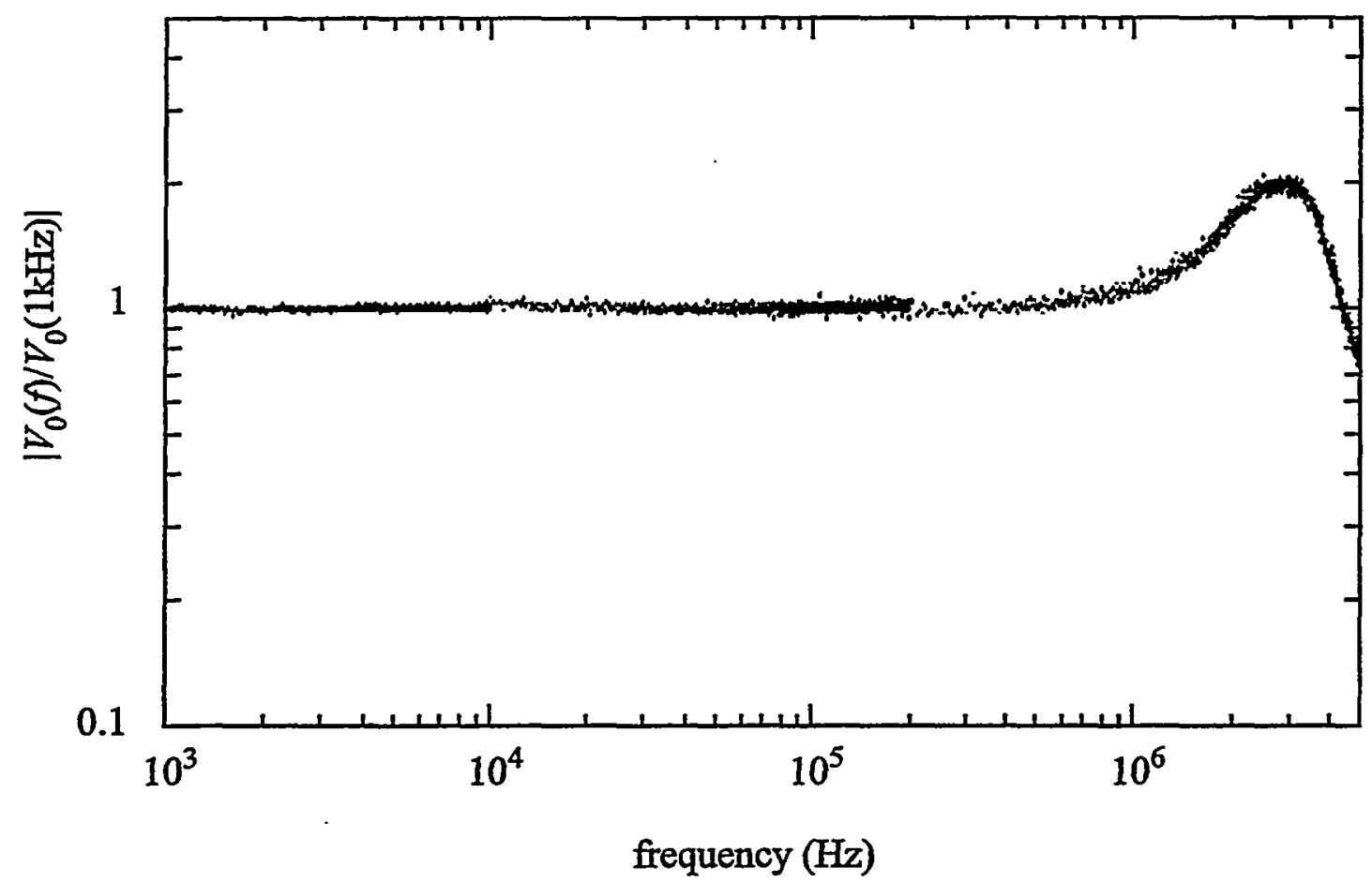

Figure 2.7: Closed loop gain $\left|V_{0}(f) / V_{0}(1 \mathrm{kHz})\right|$ of flux locked SQUID at 4.2 vs frequency, normalized to value at $1 \mathrm{kHz}$. Data were collected in three separate frequency ranges. 
is defined as the bandwidth of the spectrometer. The gain is high if $\left(R_{f} / M_{f}\right)$ is also high; however, one must chose the value of $R_{f}$ and $M_{f}$ so that the Nyquist noise of $R_{f}$ will not couple a large flux noise into the SQUID via $M_{f}$ Also, since $G(\omega)$ is proportional to $V_{\Phi, \text { new }}$ one should maximize the APF circuit to give the best possible transfer function.

To determined the frequency response of the system, a small flux $\left(\sim \Phi_{0} / 20\right.$ peak-topeak) is applied to the SQUID and $V_{0}(f)$ is measured as a function of frequency. Figure 2.7 shows $\left|V_{0}(f) / V_{0}(1 \mathrm{kHz})\right|=|G(f) /[G(f)+1]|$ versus frequency for $M_{f}=220 \mathrm{pH}, R_{f}=5 \mathrm{k} \Omega$, $G_{a}=150, \tau_{i}=26 \mathrm{~ns}$, and $V_{\Phi}=300 \mu \mathrm{V} / \Phi_{0}$. We see that the response is very nearly flat to $1 \mathrm{MHz}$ with a peak at about $3 \mathrm{MHz}$. The $3 d B$ point is at approximately $5 \mathrm{MHz}$. The calculated frequency at which $|G|=1$ is $5.9 \mathrm{MHz}$, in quite good agreement with the measured frequency.

Figure 2.8 shows the voltage noise at the output of the flux-locked loop divided by $\Delta V_{0} / \Phi_{0}$ at $1 \mathrm{kHz} ; \Delta V_{0}\left(=R_{f} / M_{f}\right)$ is the change in output voltage produced by the application of one flux quantum to the SQUID, and ranges from $50 \mathrm{mV}$ to $250 \mathrm{mV}$. The flux noise is approximately white, with a value of $3.5 \mu \Phi_{0} \mathrm{~Hz}^{-1 / 2}$, from $1 \mathrm{kHz}$ to $1 \mathrm{MHz}$; $1 / f$ noise from the preamplifier becomes dominant below about $100 \mathrm{~Hz}$. There is a peak near $2 \mathrm{MHz}$ associated with the peak in the gain in Figure 2.8.

It should be mentioned that using a CLC 425 for the second preamplifier stage tends to be less stable than an AD 797. However, since the CLC 425 has a very high GBW, it is still a very useful component if the instability can be worked out. Most of my measurements, however, were done with the $\mathrm{AD} 797$ in the second stage. In the latest electronic design, the SQUID box contains two AD797 amplifiers after the preamplifier, 


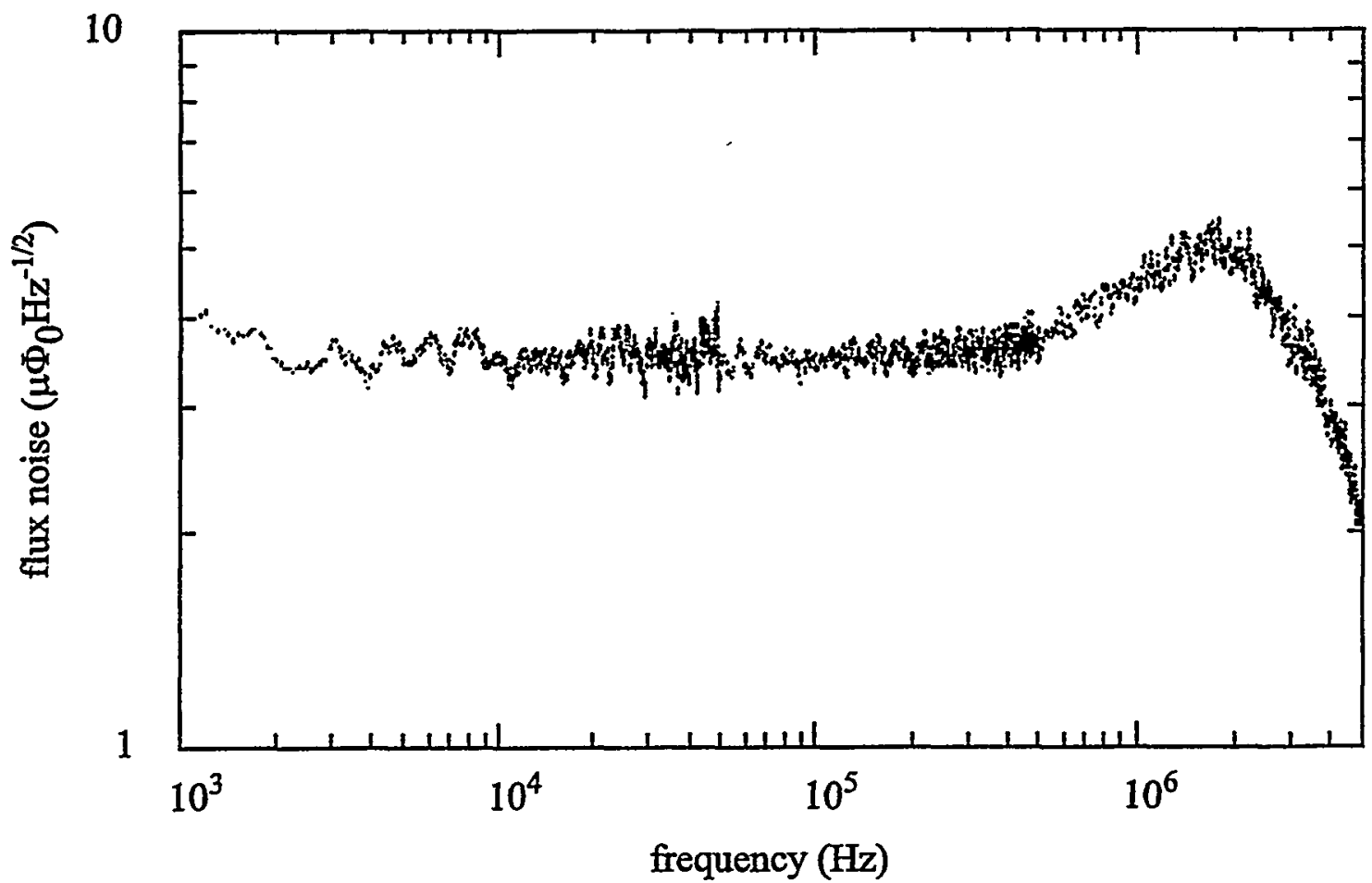

Figure 2.8: Flux noise of flux locked SQUID at 4.2 K. Data were collected in three separate frequency ranges.

providing a large, stable gain for the flux locked loop.

\subsection{Spectrometer}

Figure 2.09 is a block diagram of the spectrometer, which, apart from minor modifications, is similar to that used previously [1]. The SQUID probe part of the spectrometer contains a cell on which different coil forms such as the static field, field pulse, and pickup coil are wound. The figure shows the cell for NQR application; I will, however, later discuss different cells used for specific experiments. The cell holding the sample is enclosed in a cylindrical superconducting shield and the dewar is surrounded by two concentric $\mu$ metal shields. 


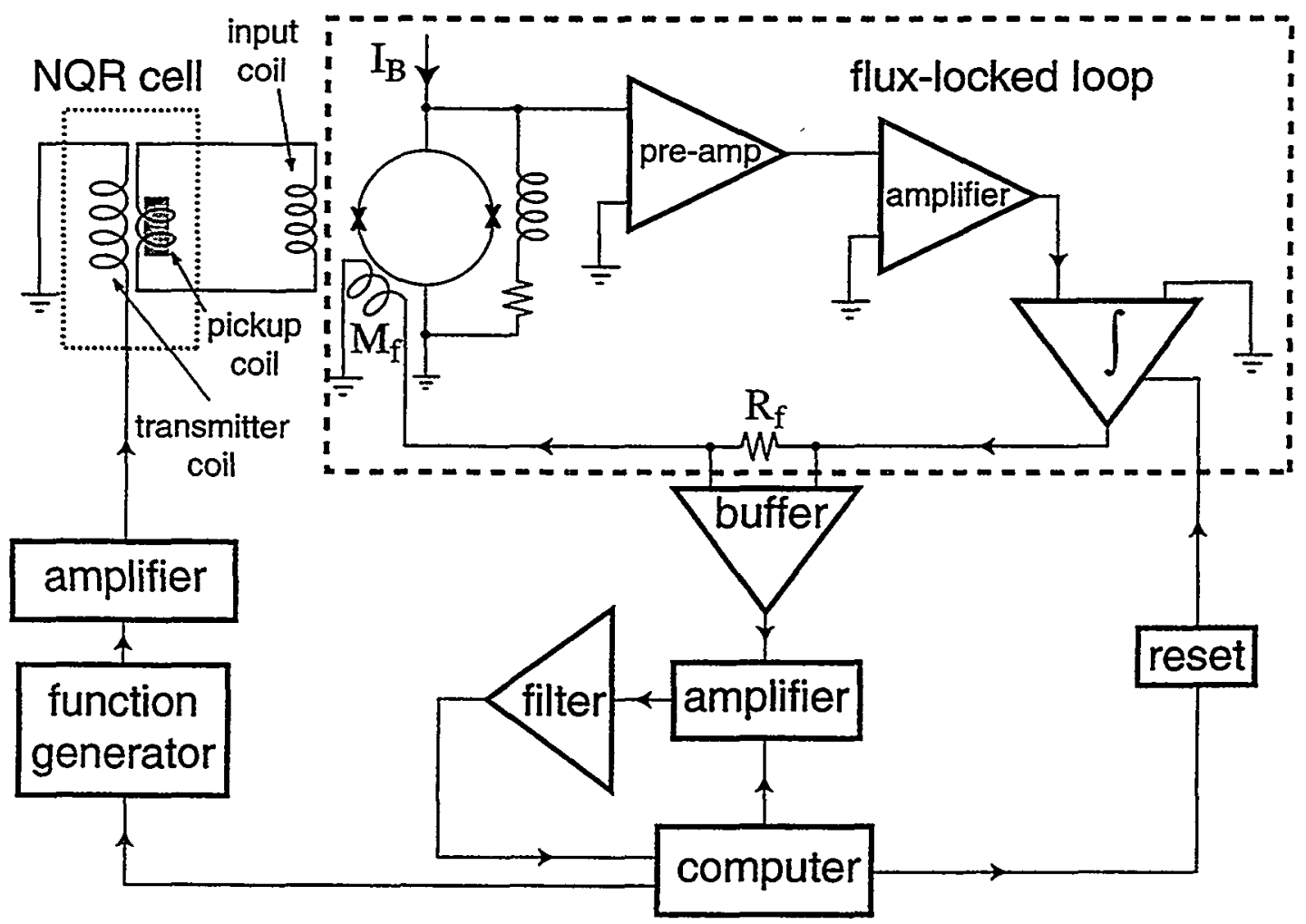

Figure 2.9: Block diagram of NQR spectrometer. Dashed lines enclose flux locked loop, and dotted lines the NQR cell

The spectrometer is controlled by a Macintosh equipped with a Techmag pulse programmer that controls the pulse sequences, turns the flux-locked loop on and off, and acquires the data. Triggered by the computer, a function generator (HP 3314A) produces a pulse sequence between 1 and 30 cycles, starting at zero phase. The pulse is amplified by an ENI 1040L (10 kHz-500kHz) amplifier and is coupled to the transmitter coil via a low pass filter and four stages of crossed diodes. These diodes present a high impedance to the transmitter coil when the pulse is turned, off, thus minimizing the noise coupled in the SQUID during the measurement. During this large audio-frequency (a.f) pulse, a photocoupled analog switch (Siliconix DG308) is triggered to short out the integrator, thereby 


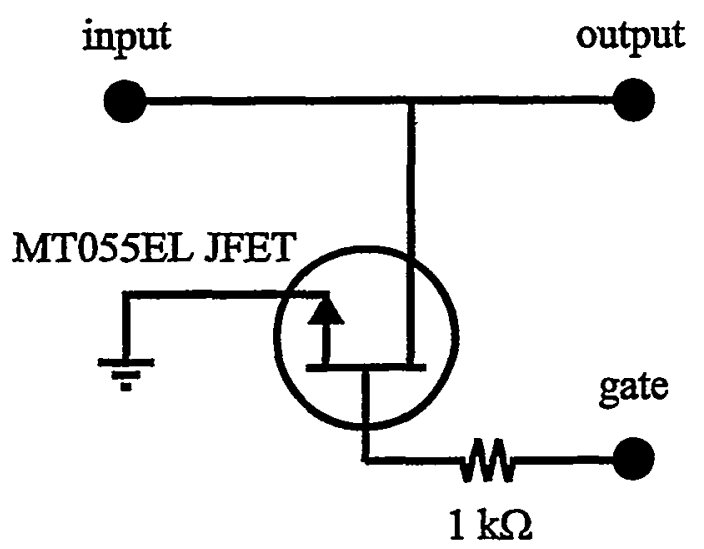

Figure 2.10: JFET switch for zeroing the unwanted signal before amplification.

preventing it from being saturated. Even after the pulse sequence is turned off, there is some dead time before the flux-locked loop can be operated: this arises from ringing of the transmitter coil, which is not particularly well matched to the output impedance of the a.f. amplifier. For a peak-to-peak pulse at the sample of $1 \mathrm{mT}$, the dead time is about $60 \mu \mathrm{s}$ at $50 \mathrm{kHz}$ and less than $10 \mu \mathrm{s}$ at $500 \mathrm{kHz}$ or higher. At the end of this dead time, the fluxlocked loop is enabled and the FID flux signal is coupled into the SQUID via the superconducting flux transformer. The output of the SQUD electronics is band pass filtered, gated with a JFET switch (Figure 2.10), and amplified. The data is acquired using a Techmag for frequencies below $200 \mathrm{kHz}$, and a digital oscilloscope for higher frequencies; the data are subsequently transferred to a computer. The JFET switch zeros out all the signal that is not part of the FID which can saturate the amplification stage, mainly the remaining transient from the af pulses. 


\section{References}

[1] N. Q. Fan, Ph. D. Thesis, University of California, Berkeley, California.

[2] D. Drung, R. Cantor, M. Peters, H. J. Scheer, and H. Koch, Appl. Phys. Lett. 57, 506 (1990)

[3] D. Drung, R. Cantor, M. Peters, T. Ryhanen, and H. Koch, IEEE Trans. Magn. MAG-27, 3001 (1991)

[4] D. Drung, H. Matz, and H. Koch, Rev. Sci. Instrum. 66, 3008 (1995).

[5] S. L. Thomasson and C. M. Gould, IEEE Trans. Appl. Supercond. 5, 3222 (1995)

[6] Dinh M. TonThat and John Clarke, Rev. Sci. Instrum. 67, 2890 (1996).

[7] John Clarke, in SQUID Sensors: Fundamentals, Fabrication and Applications, edited by H. Weinstock (Kluwer Academic, Dordrecht, the Netherlands, 1996), p. 1.

[8] B.D. Josephson, Phys. Lett. 1 (1962) 251.

[9] C. D. Tesche and John Clarke, J. Low. Temp. Phys. 27 (1977) 301.

[10] F. Wellstood, Ph. D: Thesis, University of California, Berkeley, California.

[11] M. B. Ketchen and J. M. Jaycox, Appl. Phys. Lett. 40 (1982) 736.

[12] S. Kohjiro, K. Enpuku, K. Yoshida, K. Yamafuji, Jap. J. Appl. Phys. 27, ( 1988) 1871.

[13] K. Enpuku, S. Kohjiro, K. Yoshida, J. Appl. Phys. 60 (1986) 4218. 


\section{Chapter 3}

\section{NMR of Laser-polarized Xenon at $4.2 \mathrm{~K}$}

\subsection{Introduction}

Xenon is one of the most intensively studied nuclei. ${ }^{129} \mathrm{Xe}$, with a nuclear spin of $1 / 2$, is used as a sensitive probe of the local environment because of its chemical inertness, its large polarizability, and its relative ease of detection by typical high field NMR[1]. Distortion of the diffuse electronic cloud due to collisions with its surroundings leads to a large detectable chemical shift of the xenon nuclear spin. The ${ }^{129} \mathrm{Xe}$ chemical shift is, typically about $100 \mathrm{ppm}[2]$ and can be easily measured in a high field NMR experiment; however, the shift is a minuscule $10 \mathrm{~Hz}$ in a $100 \mathrm{kHz}$ Larmor line. Such a measurement is not realistic, given the typical linewidth of the ${ }^{129} \mathrm{Xe}$ lines, even with a SQUID.

However, one special and important property of ${ }^{129} \mathrm{Xe}$ is that its nuclear polarization can be easily increased by 5 orders of magnitude over that of thermal equilibrium at room temperature and 4 tesla through a process called spin exchange, which takes place 
via Fermi contact interaction between xenon nuclei and optical pumped rubidium atoms[3-5]. The increase of the ${ }^{129} \mathrm{Xe}$ NMR signal by this "hyperpolarization" has made possible a number of novel experiments, among them enhanced magnetic resonance imaging (MRI) of organisms [6-10] and enhanced NMR of surfaces and solutions [11-13]. This type of enhancement is feasible, provided a significant fraction of the ${ }^{129} \mathrm{Xe}$ polarization can be transferred to the spins of the nuclei interest. A considerable body of current research is aimed at transferring the xenon polarization to systems in which the signal-tonoise ratio is very small, such as low spin-density species and surface spin species. In view of its function as an NMR/MRI contrast agent, a spin-polarization source for cross polarization [14-16], and a potential low-temperature NMR/NQR matrix [17], previous investigations have addressed the question of ${ }^{129} \mathrm{Xe}$ relaxation in gas [18], solution [19], and solid phases [20-22]. Of particular relevance for the long term storage of this hyperpolarized xenon at low temperatures is the decay of polarization via spin-lattice relaxation as the magnetic field is reduced towards zero. Gatzke et al. [22] reported the ${ }^{129} \mathrm{Xe}$ spin-lattice relaxation time, $T_{1}$, at $4.2 \mathrm{~K}$ in magnetic fields ranging from $0.2 \mathrm{~T}$ to $5 \mathrm{mT}$, corresponding to ${ }^{129} \mathrm{Xe}$ Larmor frequencies of $2 \mathrm{MHz}$ to $50 \mathrm{kHz}$. They found that $T_{1}$ depends strongly on magnetic field in this range.

This work is an extension of that experiment. Using the SQUID NMR/NQR spectrometer, ${ }^{129} \mathrm{Xe}$ NMR has been observed down to $200 \mathrm{~Hz}$, near the xenon dipole-dipole interaction[23]. In the first part of this chapter, I will discuss general NMR theory; then I spend some time on the spin polarization transfer during an optical pumping process. Finally, I present some the results. 


\subsection{NMR Theory}

A year ago, I was at a symposium at Stanford celebrating the 50 years that have passed since the first detection of nuclear magnetic resonance by Bloch[24]. Now, NMR has become a routine tool in science, especially chemistry and biology. Chemical shift information in an NMR experiment, discovered more than 40 years ago by Proctor and Yu[25], is still a standard and powerful method of structural determination of chemical compounds. Multi-dimensional NMR methods [26] extended the technique further to determine even more complicated molecules such as proteins, as well as to provide dynamical studies of materials. With the application of MRI in medicine, NMR has become a common tool for enhancing the quality of our lives and thus MRI has become an everyday word. "Tomorrow, he will get an MRI checkup" explained the announcer during a ball game, when a player got injured. All of these applications ensure the practicality and usefulness of NMR for years to come.

NMR can be performed on any nuclei with a non-zero magnetic moment $(I \neq 0)$. The wealth of information obtained from an NMR experiment arises from the presence of many different types of interactions acting on the nuclear spins, such as the interaction due to an external magnetic field $\left(H_{\mathrm{ext}}\right)$, the dipole interaction due to neighboring spins $\left(H_{\text {dipole-dipole }}\right)$, the electric quadrupole interaction due to the presence of the quadrupole moment of the surrounding electron cloud ( $\left.H_{\text {quadrupole }}\right)$, etc... In the most general form, the Hamiltonian can be written as the sum of all these interactions:

$$
H=H_{\text {ext }}+H_{\text {dipole-dipole }}+H_{\text {quadrupole }}+\ldots .
$$

For solid ${ }^{129} \mathrm{Xe}$, the dominant term is the interaction due to an external magnetic 


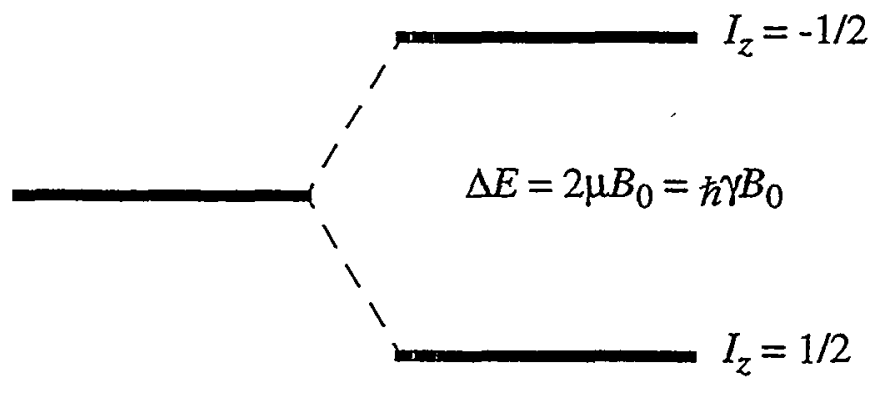

$$
B_{0}=0 \quad B_{0} \neq 0
$$

Figure 3.1: Energy level splitting of a nucleus of spin $I=1 / 2$ in the presence of an applied $B_{0}$.

field $\boldsymbol{B}_{0}$, and the Hamiltonian reduces to

$$
H=-\mu \cdot B_{0}
$$

where $\mu$ is the magnetic dipole moment of the nuclei. Quantum mechanically, $\mu=\hbar \gamma I$, where $\gamma$ is the gyromagnetic ratio (a constant specific to a given nuclei) and $I$ is the nuclear spin quantum number. Thus Eqn 3.1 can be rewritten as

$$
\mathrm{H}=-\hbar \gamma \boldsymbol{I} \cdot \boldsymbol{B}_{0}=\hbar \gamma I_{z} B_{0},
$$

assuming $B_{0}=B_{0} \hat{z}$; here, $I_{z}$ is the $z$ component of the spin $\left(-I \leqslant I_{z} \leqslant I\right)$. The energy levels of the nucleus with $I=1 / 2$, like ${ }^{129} \mathrm{Xe}$ or ${ }^{1} \mathrm{H}$, in the presence of $B_{0}$, are split into two energy levels as shown in Fig 3.1. In thermal equilibrium, the populations of these two energy levels are different, leading to a net $z$ magnetization $M_{z}$ in a system of $N$ spins of

$$
M_{z}=N_{\hbar} \gamma P
$$

where $P$ is the polarization; $P$ is equal to $\tanh \left(\hbar \gamma B_{0} / 2 k_{\mathrm{B}} T\right)$ and approximately equal to 

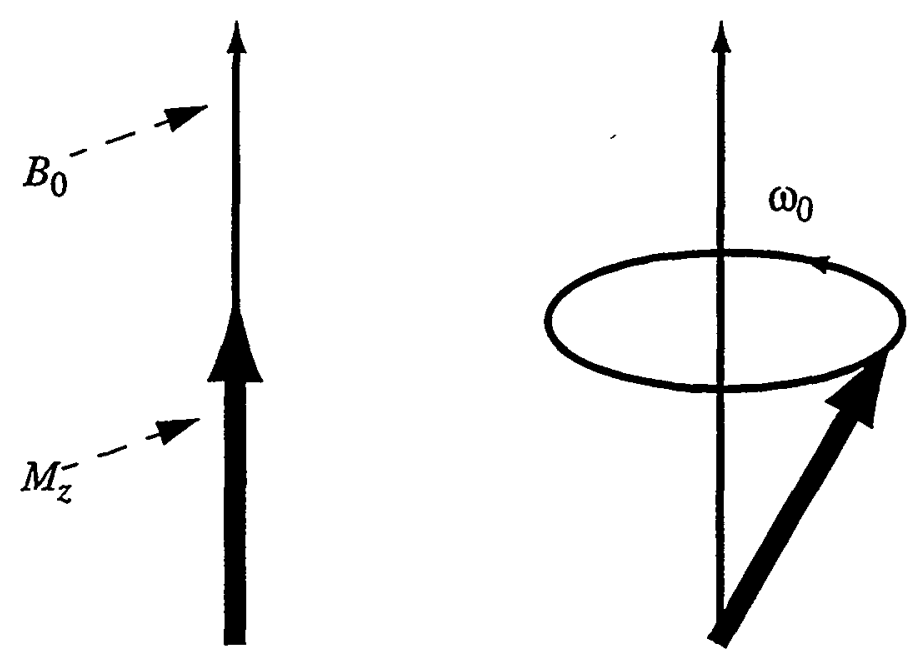

Figure 3.2: In thermal equilibrium, the magnetization aligns with the applied $B_{0}$ field. When a pulsed magnetic field $B_{r f}$ is applied to the sample, the magnetization is tipped, producing a rotating detectable component along the $x$ and $y$ plane.

$\hbar \gamma B_{0} / 2 k_{\mathrm{B}} T$, for $\hbar \gamma B_{0} \ll k_{\mathrm{B}} T$. One way to detect the difference in the energy levels is by irradiating the sample with a transverse radio frequency $(r f)$ field of the right frequency $\left(\omega_{0}=\gamma B_{0}\right)$, to equalize the population, and then to look for the changes in this magnetization. This method is called $c w$ NMR, where $c w$ means continous wave, and is typical of early experiments. However, a more powerful and common way is by pulsing the sample with a short transverse $r f$ field $B_{r f}$ at carrier frequency $\omega$ near $\omega_{0}$ to convert the $z$ magnetization into detectable $x$ and $y$ components; here $B_{r f}=2 B_{1} \cos \omega t \hat{x}$. This $x$ and $y$ magnetization precess around $B_{0}$ with angular frequency $\omega_{0}$, Figure 3.2 , and can be measured by studying the emf induced in a fixed detection coil. Since the change of $z$ magnetization measured by $c w$ technique is much slower than $\omega_{0}$, the pulsed technique offers a larger 
detectable signal and is the preferred technique of modern NMR.

After being disturbed from equilibrium, the $z$ magnetization of the spins will be restored after some time $T_{1}$ through the interaction of the spin with the lattice. This time $T_{1}$ is called the longitudinal relaxation time or the spin-lattice relaxation time. The $x$ and $y$ magnetizations will also return to their equilibrium values of zero after some time $T_{2} . T_{2}$ is a measure of the time during which the precessing spins that contribute to $x$ and $y$ component of the magnetization stay in phase with respect to each other. Since each spin experiences a slightly different field at its own position, $T_{2}$ is dependent on the interaction of the spins with the fluctuating "local fields" and is known as the transverse relaxation time or spin-spin relaxation time.

The equations that govern the motion of the magnetizations, the Bloch equations, are expressed below:

$$
\begin{aligned}
& \frac{d M_{x}}{d t}=\gamma M_{y} B_{0}+\gamma M_{z} B_{1} \sin \omega t-\frac{M_{x}}{T_{2}}, \\
& \frac{d M_{y}}{d t}=-\gamma M_{x} B_{0}+\gamma M_{z} B_{1} \cos \omega t-\frac{M_{y}}{T_{2}}, \\
& \frac{d M_{z}}{d t}=-\gamma M_{x} B_{1} \sin \omega t+(-\gamma) M_{y} B_{1} \cos \omega t-\frac{M_{z}-M_{0}}{T_{1}} .
\end{aligned}
$$

These Bloch equations can be transfromed into a frame rotating around $B_{0}$ at a frequency $\omega$, reducing to the much simpler equations

$$
\frac{d \tilde{M}_{x}}{d t}=\Delta \omega \tilde{M}_{y}-\frac{\tilde{M}_{x}}{T_{2}}
$$




$$
\begin{aligned}
& \frac{d \tilde{M}_{y}}{d t}=-\Delta \omega \tilde{M}_{x}+\gamma \tilde{M}_{z} B_{1}-\frac{\tilde{M}_{y}}{T_{2}}, \\
& \frac{d \tilde{M}_{z}}{d t}=-\omega_{1} \tilde{M}_{y}-\frac{\tilde{M}_{z}-M_{0}}{T_{1}},
\end{aligned}
$$

where $M_{0}$ is the initial $z$ magnetization, $\Delta \omega=\omega_{0}-\omega$, and $\tilde{M}_{i}$ is the magnetization in the rotating coordinate frame $(i=x, y, z)$. The general solution of these equations consist of two parts: the steady state solution and the exponentially decreasing solution. In the steady state, the "rotating frame" transverse magnetization is dependent on the initial value of $M_{0}$, and when transferred back to the lab frame the transverse component is time dependent and induces a detectable signal.

\subsection{Optical Pumping and Spin Exchange}

Even though NMR is a powerful technique, it is very insensitive when compared to other spectroscopies such as X-ray or electron spin resonance because the NMR signal is small to begin with. The magnitude of an NMR signal is proportional to the polarization $P$ and in a typical high field magnet of 7 Tesla (proton Larmor line of $300 \mathrm{MHz}$ ), the ${ }^{129} \mathrm{Xe}$ polarization is tiny, roughly $10^{-5}$. One way to increase the signal is to go to a yet higher field since the detected signal obtained by an induction method is approximately proportional to $Q \omega^{2}$, where $Q$ is the circuit quality factor. State-of-the-art superconducting magnets are limited to a field of 23 tesla or a proton Larmor line of $1 \mathrm{GHz}$. Such magnets are not only very expensive but also difficult to produce. Looking at the dependence of $P$ on $T$, we see that signals also increase if the experiments are done at low temperature. 
However, there are limitation to the kind of experiments accessible at or below $4.2 \mathrm{~K}$. Another way to generate a large, non-thermal equilibrated population from the very beginning, for example, optical pumping. The typical polarization obtained in an optical pumping experiment is about $10 \%(0.1)$, which is a 4 orders-of-magnitude increase in signal compared to that from an equilibrium population. Achieving hyperpolarized xenon spins involves a two step process. First, the electrons of an atomic system of some alkali metal are polarized with circularly polarized laser light; second the polarized electronic state of the alkali metal is transferred to the xenon gas through a spin-exchange interaction.

\subsubsection{Optical Pumping}

The idea that a nonequilibrium population of spin energy levels can be achieved was first discovered by Alfred Kastler in 1949[27], and for that he won the 1966 Nobel Prize in Physics[28]. This optical pumping method relies on the transfer of angular momentum from the polarized light to the electrons in an atomic system. The basic idea of optical pumping can be understood by a hypothetical atom with the energy level diagram shown in Figure 3.3. The ground state is ${ }^{2} \mathrm{~S}_{1 / 2}$ and the first excited state ${ }^{2} \mathrm{P}_{1 / 2}$. In the presence of a magnetic field, the ${ }^{2} \mathrm{~S}_{1 / 2}$ and ${ }^{2} \mathrm{P}_{1 / 2}$ energy levels become non-degenerate. Quantum mechanics dictates a set of selection rules in which electronic transitions can take place, namely $\Delta \ell=1, \Delta m=0, \pm 1$, where $\ell$ is the orbital quantum number, and $m$ is its $z$ projection. However, because of conservation of angular momentum with the incoming photon, only $\Delta m=-1$ is allowed for right circularly polarized light. In the beginning, the system in thermal equilibrium has almost the same number of spins up and spins down in 
(a)

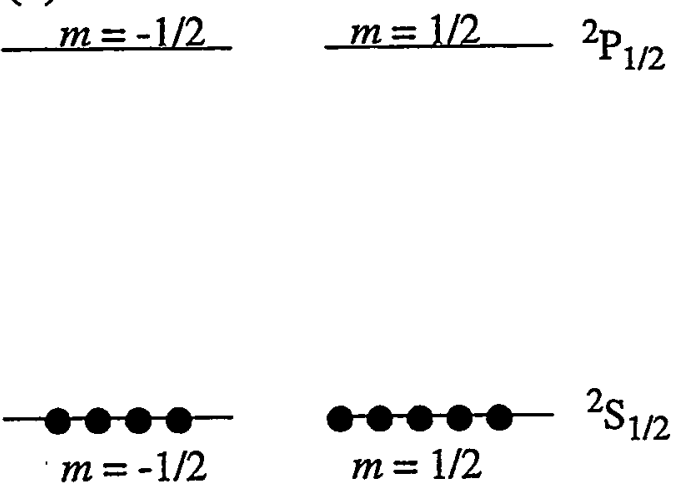

(b)

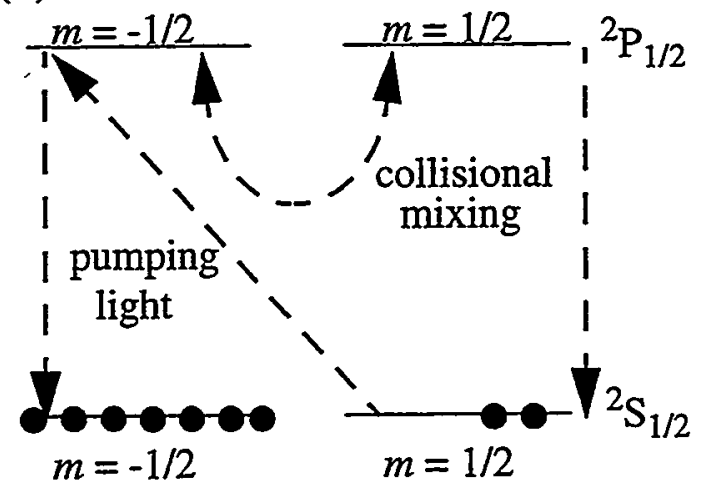

Figure 3.3: (a) At thermal equilibrium, the two ${ }^{2} \mathrm{~S}_{1 / 2}$ levels are nearly equally populated. (b) The effect of interaction with a left circularly polarized light.

the ground ${ }^{2} \mathrm{~S}_{1 / 2}$ state, Figure 3.3(a). When right circularly polarized light is applied, it excites the electrons from the $m=1 / 2$ of the ${ }^{2} \mathrm{~S}_{1 / 2}$ state to the $m=-1 / 2$ of the ${ }^{2} \mathrm{P}_{1 / 2}$ state. At the typical pressure of an optical pumping experiment (roughly $1 \mathrm{~atm}$ ), collisions with noble-gas atoms rapidly mix the sublevel population of the excited state, Figure 3.3(b). The excited state is also quenched by a small amount of $\mathrm{N}_{2}$ gas present, which also eliminates radiation trapping as a source of relaxation[29]. In the ${ }^{2} \mathrm{P}_{1 / 2}$ state, the electron lifetime is short and it decays back down to the ${ }^{2} S_{1 / 2}$ level, with same probability of $1 / 2$ for both the $m=1 / 2$ and the $m=-1 / 2$ state. Thus atoms, which have $-1 / 2$ unit of angular momentum before the absorption of a photon, will have a net average of 0 unit of angular momentum after collision; the net gain is $1 / 2$ unit of angular momentum, the remainder is lost to translational motion[29]. This process is repeated again and again many times during the pumping process, and assuming the lifetime of ground state is sufficiently long, the electron polarization is gradually built up.

The electronic states of the alkali metals make them a good system for optical 
pumping, and rubidium is used. Both isotopes of rubidium $\left({ }^{85} \mathrm{Rb}[I=5 / 2,72.12 \%]\right.$ and ${ }^{87} \mathrm{Rb}$ $[I=3 / 2,27.85 \%])$ possess a ${ }^{2} \mathrm{~S}_{1 / 2}$ ground state and ${ }^{2} \mathrm{P}_{1 / 2}$ excited state. The lifetime in the excited state is about 30 nanoseconds, allowing a short pumping time, and the long $T_{1}$ of the polarized electronic population, 1 second, allowing a sufficient time for spin exchange to the xenon nuclei. The splitting between the ground state ${ }^{2} \mathrm{~S}_{1 / 2}$ and the state ${ }^{2} \mathrm{P}_{1 / 2}$ is about $794.7 \mathrm{~nm}$, which makes it accessible with a current commercial diode laser. With a melting point around $40{ }^{\circ} \mathrm{C}$, the rubidium vapor density can be easily controlled at modest temperatures where chemical interaction with the glass container is not a problem[29].

\subsubsection{Spin Exchange}

Once the electronic state of the rubidium atoms is polarized, we can use them to spin-polarize the nuclei of the xenon gas through a process called spin exchange, indicated in Figure 3.4. The first experiment was done in a system of optically pumped sodium
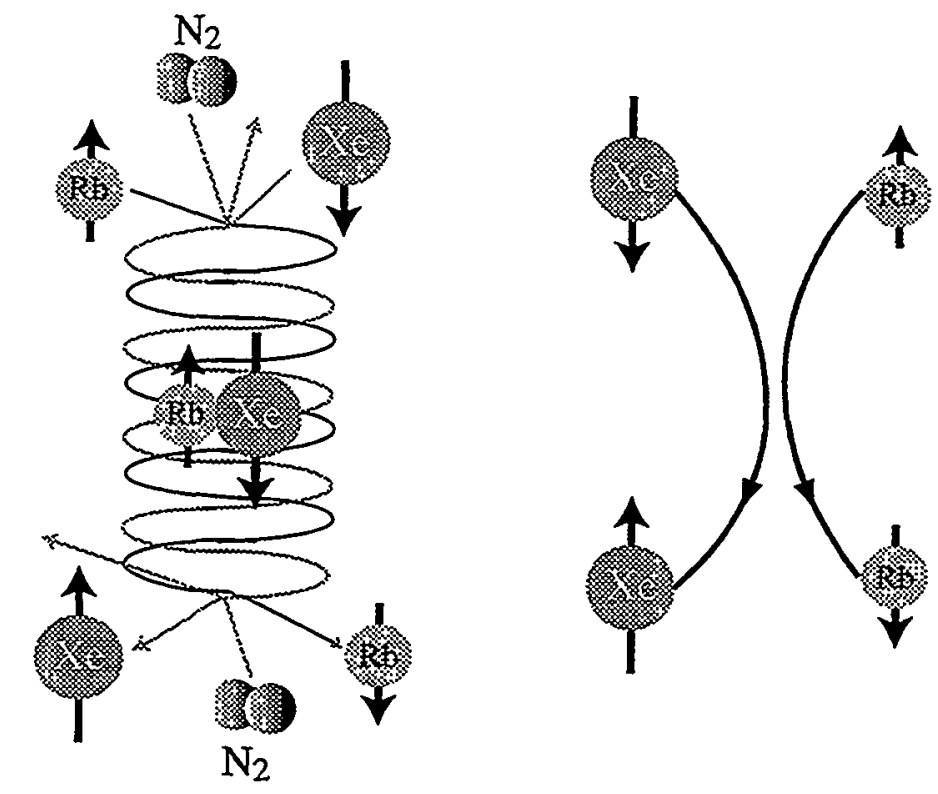

Figure 3.4: $\quad$ Spin exchange processes. (a) Formation of rubidium and xenon van de Waals molecule. (b) Binary collision between $\mathrm{Rb}$ and $\mathrm{Xe}$. 
atoms and free electrons by Dehmelt[30]. Spin exchange allows for an indirect way to polarize systems that can not be pumped directly. The transfer of the rubidium electronic polarization to the xenon nucleus has been extensively studied by Happer and coworkers[31,32]. In a system where collisions are limited, xenon and rubidium form long-lived van der Waal's molecules[33-36], enhancing the spin exchange process, Figure 3.4(a). However, most of the time, the optical pumping process is done in a high pressure of noble gas in which collisions dominate [Figure 3.4(b)], and the van der Waal's molecule is very short lived. Nevertheless, it is understood that the rubidium atom exchanges its electronic polarization with the xenon nuclear spin during this short contact. The dominant interactions controlling this spin-exchange are the Fermi contact interaction and the spin-rotational interaction, as given by

$$
\mathrm{H}=-\alpha I \cdot S+\beta N \cdot S .
$$

Here $\alpha$ is the isotropic hyperfine interaction, $\beta$ is the spin-rotational interaction coefficient, $I$ is the xenon nuclear spin, $S$ is the rubidium electron spin, and $N$ is the rotational angular momentum. The Fermi contact interaction can be rewritten as

$$
\left.\alpha(I \cdot S)=\frac{8 \pi}{3} \gamma_{e} \gamma_{X e} \mid \Psi(0)\right)\left.\right|^{2}\left[I_{z} S_{z}+\frac{1}{2}\left(I_{+} S_{-}+I_{-} S_{+}\right)\right]
$$

where $\gamma_{e}$ and $\gamma_{X e}$ represent the gyromagnetic ratios of the electron and xenon nucleus, respectively, and $\mid \Psi(0))\left.\right|^{2}$ is the probability of the electron being at at the xenon nucleus. One of the exchange terms $I_{+} S_{-}$or $I_{-} S_{+}$, depending on the polarized rubidium state, leads to the exchange of $\mathrm{Rb}$ electron spin and xenon nuclear spin. The spin rotational term on the other hand transfers the rubidium electron polarization to the translational degree of freedom of the atoms, thereby, limiting the spin exchange efficiency. 


\subsection{Experimental Setup}

The optical pumping apparatus is shown in Figure 3.5. The pumping cell, a glass cylinder with a volume of $10 \mathrm{~cm}^{3}$, is connected to the sample tube through glass transfer lines with stopcocks for gas handling. The cell is coated with a thin film of rubidium and heated by flowing hot nitrogen gas to $70-90^{\circ} \mathrm{C}$ to increase the rubidium vapor pressure for optimal absorption. During optical pumping, the cell is illuminated with a circularly polarized light from either a $1 \mathrm{~W}$ cw Titanium-Sapphire ring laser (Schwartz ElectroOptics) or a $0.5 \mathrm{~W}$ cw tunable diode laser (Spectra Diode Laboratories). The laser light is passed through a polarizer and a quarter-wave $(\lambda / 4)$ lens. The polarizer minimizes the deviation

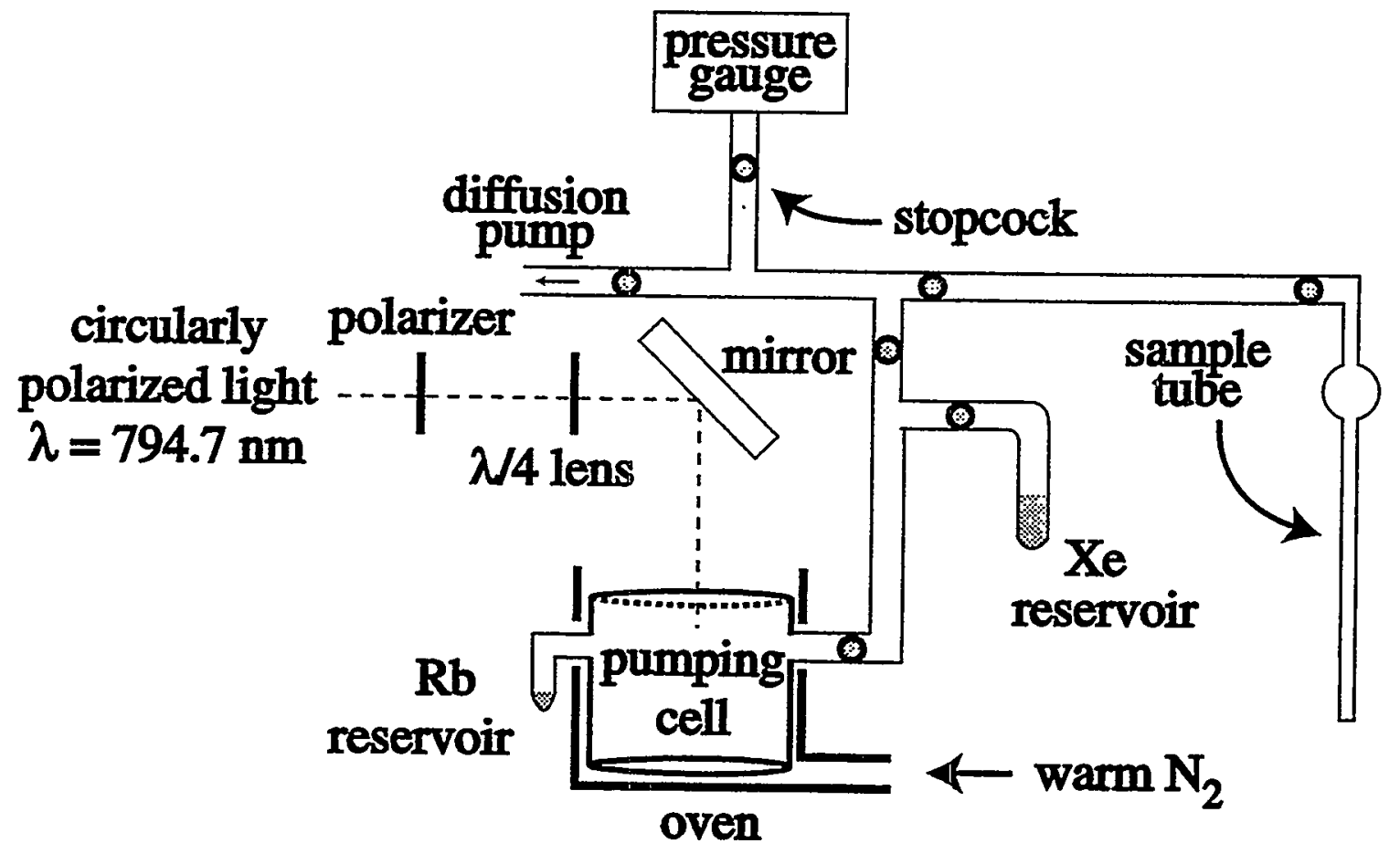

Figure 3.5: Optical pumping apparatus, consisting of a $10 \mathrm{~cm}^{3}$ cylinder glass cell surrounded by a solenoid (not shown), a $\mathrm{Rb}$ reservoir, a xenon reservoir, and a sample cell linked by glass vacuum lines provided by stopcocks. The laser light illuminates the pumping cell via a circular polarizer. 
of the laser from linear polarization and the $\lambda / 4$ lens changes the linear polarized light into circularly polarized light. The laser is tuned to the rubidium D1 optical transition, 794.7 $\mathrm{nm}$, and the fluorescence in the cell is monitored with an infrared viewer. Alternatively, a photodetector allows the determination of absorbed light by $\mathrm{Rb}$ atoms by monitoring the transmitted light passing through the cell.

Xenon gas is first loaded and stored in the side arm (Xe reservoir) that contains about $2 \mathrm{~cm}^{3}$ of $\mathrm{Rb}$. Since $\mathrm{Xe}$ gas can be contaminated by $\mathrm{O}_{2}$ from run to run, the xenon gas is cleaned in this sidearm, by heating the $\mathrm{Rb}$ to react with any $\mathrm{O}_{2}$. This process is repeated a few times before the Xe gas is loaded into the pumping cell. "Dirty" xenon will oxidize the $\mathrm{Rb}$ in the pumping cell and will reduce the pumping efficiency as the $\mathrm{Rb}$ oxide, which adheres to the inside of the cell, scatters the incoming laser light. Typically, $2 \mathrm{mM}$ of xenon is admitted into the pumping cell and irradiated for 30 minutes. A magnetic field of $3 \mathrm{mT}$ splits the $\mathrm{Rb}$ and Xe levels during optical pumping. Subsequently, the cell is cooled to below $40{ }^{\circ} \mathrm{C}$ to lower the rubidium vapor pressure, and allow the xenon gas to flow into the sample tube. The xenon freezes at the tip of this tube, which is immersed in liquid nitrogen, in the presence of a $0.1 \mathrm{~T}$ magnetic field to keep the xenon polarization from relaxing. Finally, the sample is inserted into the SQUID spectrometer. A solenoid around the top of the cryostat maintains the $\mathrm{Xe}$ in a $10 \mathrm{mT}$ magnetic field during the transfer process, except for an interval of a second or less when the tube is lowered into the coils. We used both isotopically enriched xenon $\left(80 \%{ }^{129} \mathrm{Xe}, 2 \%{ }^{131} \mathrm{Xe}, \mathrm{EG} \& \mathrm{G}\right.$ Mound) and naturally abundant xenon $\left(26.4 \%{ }^{129} \mathrm{Xe}, 21.2 \%{ }^{131} \mathrm{Xe}\right)$. The enhanced polarization of the enriched sample was estimated to be $10 \%$ before it was lowered into the SQUID spectrometer. This is measured by comparing the signal of the optically pumped ${ }^{129} \mathrm{Xe}$ to a 


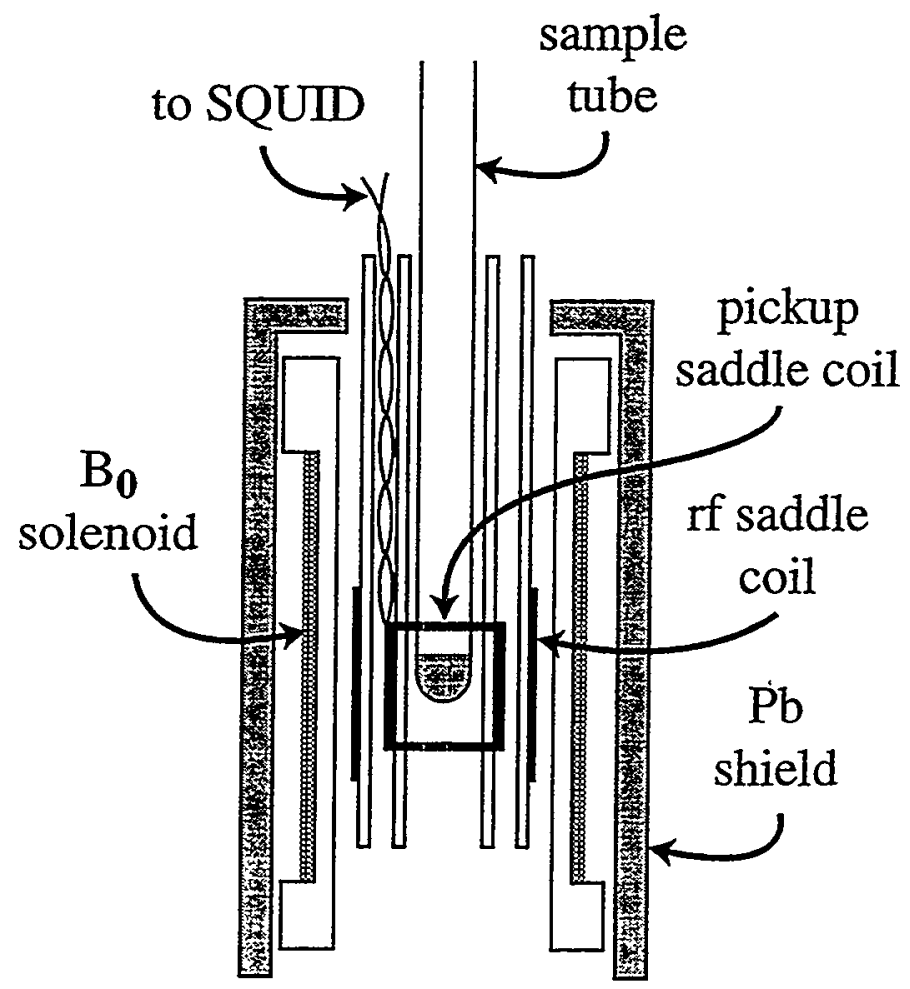

Figure 3.6: Schematic drawing of the SQUID-based NMR cell. The sample is at the center of the orthogonal pickup and excitation saddle coils; a persistent current solenoid provides a static magnetic field along the axis of the sample-tube. Coils and sample are enclosed in a $\mathrm{Pb}$ tube. Two $\mu$-metal shields (not shown) reduce the ambient magnetic field to below $0.1 \mu \mathrm{T}$.

standard containing $2.5 \mathrm{~atm}$ of enriched ${ }^{129} \mathrm{Xe}$ mixed with 100 torr of $\mathrm{O}_{2}$, used to reduce the spin lattice relaxation time of Xe and allowed for signal averaging.

Figure 3.6 shows schematically the SQUID cell used in this experiment; the cell is typically of the one we use for SQUID NMR experiments. The signal is detected by a saddle coil, with two turns on each side, connected to the 50-turn input coil of the SQUID. This input circuit is entirely superconducting, and thus responds to magnetic flux at frequencies down to zero. A second saddle coil provides audio frequency (af) excitation and 
is mounted orthogonally to the pickup coil. This ensures that little of the af pulse is picked up by the flux transformer during pulsing, which can result in a long dead time. A superconducting solenoid with a thermal switch enabling it to be operated in a persistent current mode provides a static field $B_{0}$ perpendicular to the axes of the saddle coil and the SQUID pickup coil. The coils are enclosed in a lead shield and the entire assembly is immersed in liquid ${ }^{4} \mathrm{He}$. The cryostat is surrounded with two $\mu$-metal shields to reduce the ambient magnetic field to below $0.1 \mu \mathrm{T}$. The tube containing spin-polarized xenon is inserted into the center of the coil.

\subsection{Results and Discussion}

Figure 3.7 shows a typical FID from solid ${ }^{129} \mathrm{Xe}$, taken with a single pulse to pro-

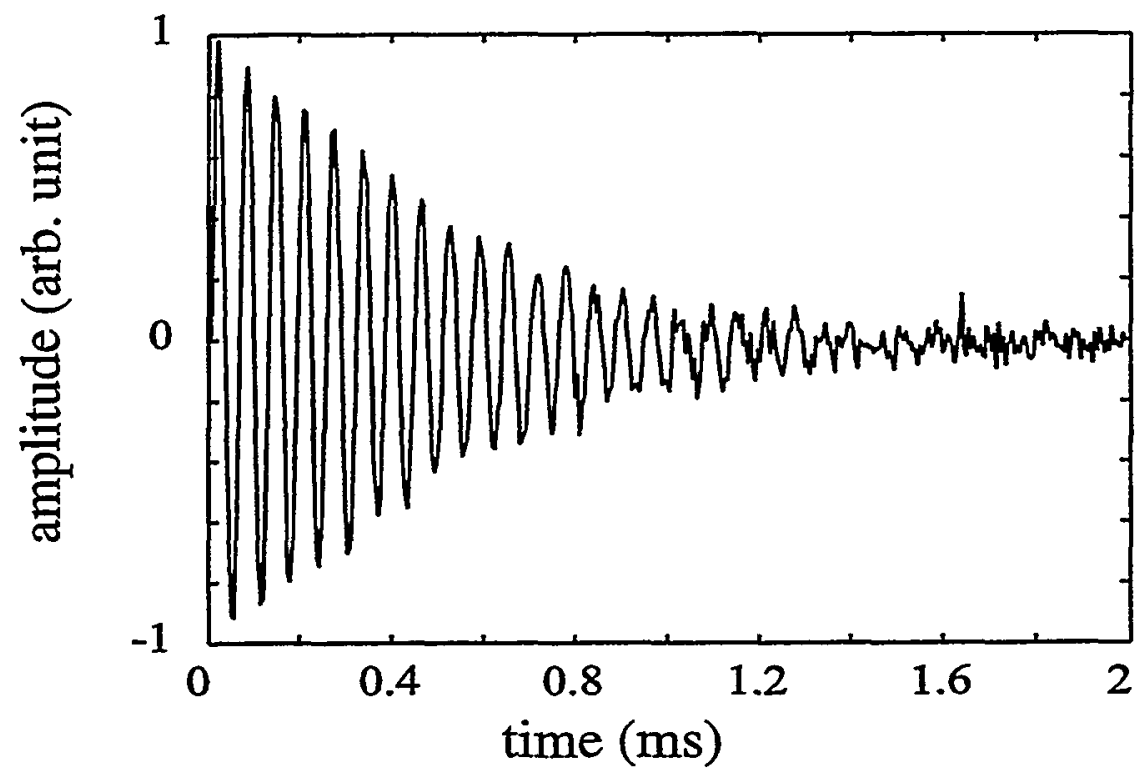

Figure 3.7: Free induction decay (FID) of an optically pumped solid ${ }^{129} \mathrm{Xe}$. Data was taken after a single pulse with a carrier frequency of $15.5 \mathrm{kHz}$. Data collected after several pulses in rapid succession give tipping angle of $8^{\circ}$. The resonance of this FID is at about $15.7 \mathrm{kHz}$ 
duce a tipping angle about $8^{\circ}$. For this specific FID, the af pulse length is 1 cycle at 15.5 $\mathrm{kHz}$ or about $65 \mu \mathrm{s}$. The Larmor line is about $15.7 \mathrm{kHz}$, corresponding to a field of $1.3 \mathrm{mT}$. At frequencies of $10 \mathrm{kHz}$ or lower, the ENI-1040L if amplifier does not amplify efficiently, and instead we used an af pulse consisting of $1 / 2$ of cycle at $10-25 \mathrm{kHz}$. Figure 3.8 shows six ${ }^{129}$ Xe NMR spectra obtained at fields ranging from $8.9 \mathrm{mT}$ down to 0.019 $\mathrm{mT}$ with corresponding ${ }^{129} \mathrm{Xe}$ Larmor frequencies of $110 \mathrm{kHz}$ to $200 \mathrm{~Hz}$. These spectra
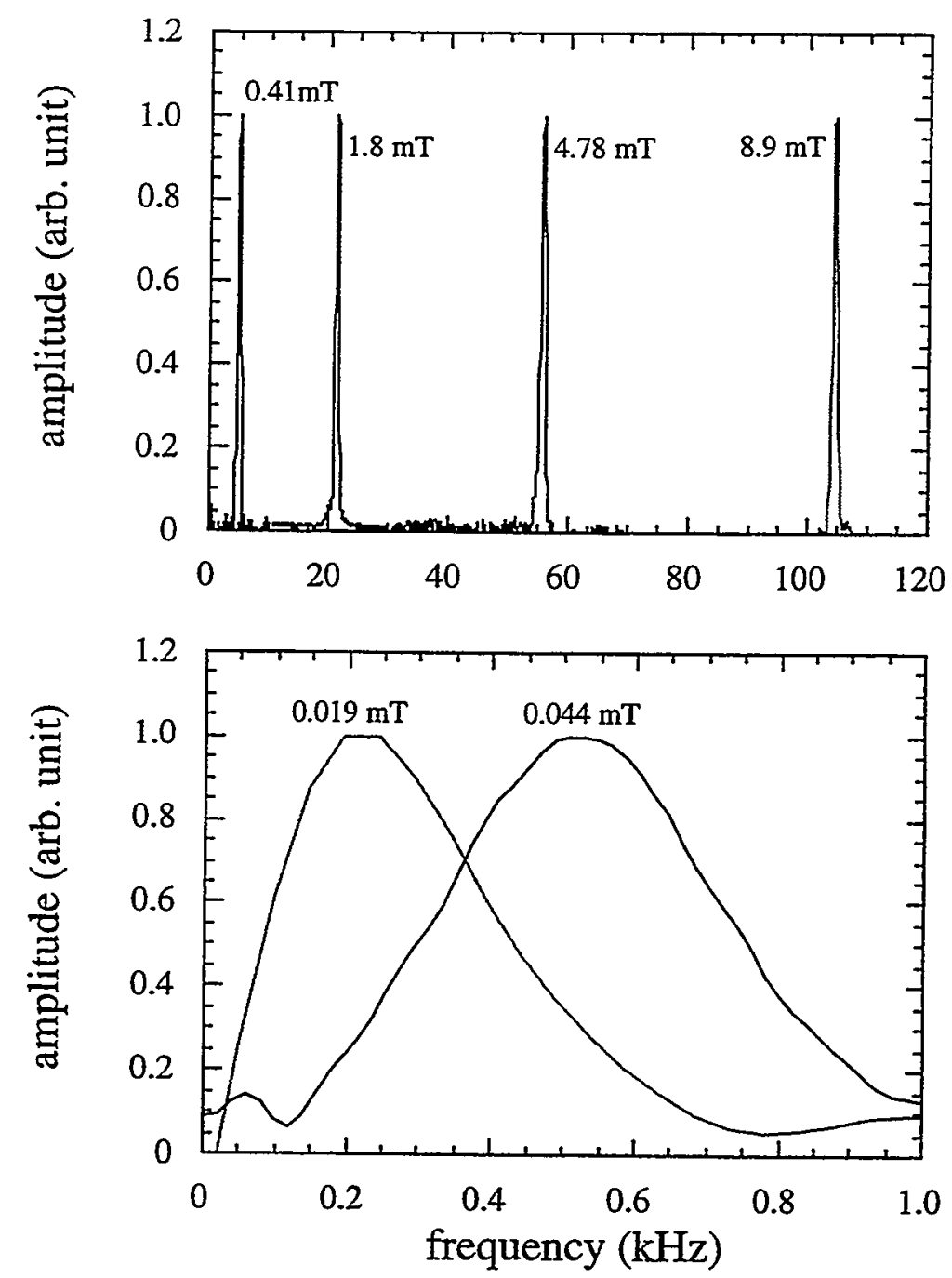

Figure 3.8: $\quad$ SQUID-detected ${ }^{129} \mathrm{Xe}$ NMR spectra from (a) isotopically enriched ${ }^{129} \mathrm{Xe}$ at 4 different magnetic fields, and (b) isotopically enriched sample at $0.044 \mathrm{mT}$ and naturally abundant sample at $0.019 \mathrm{mT}$. 


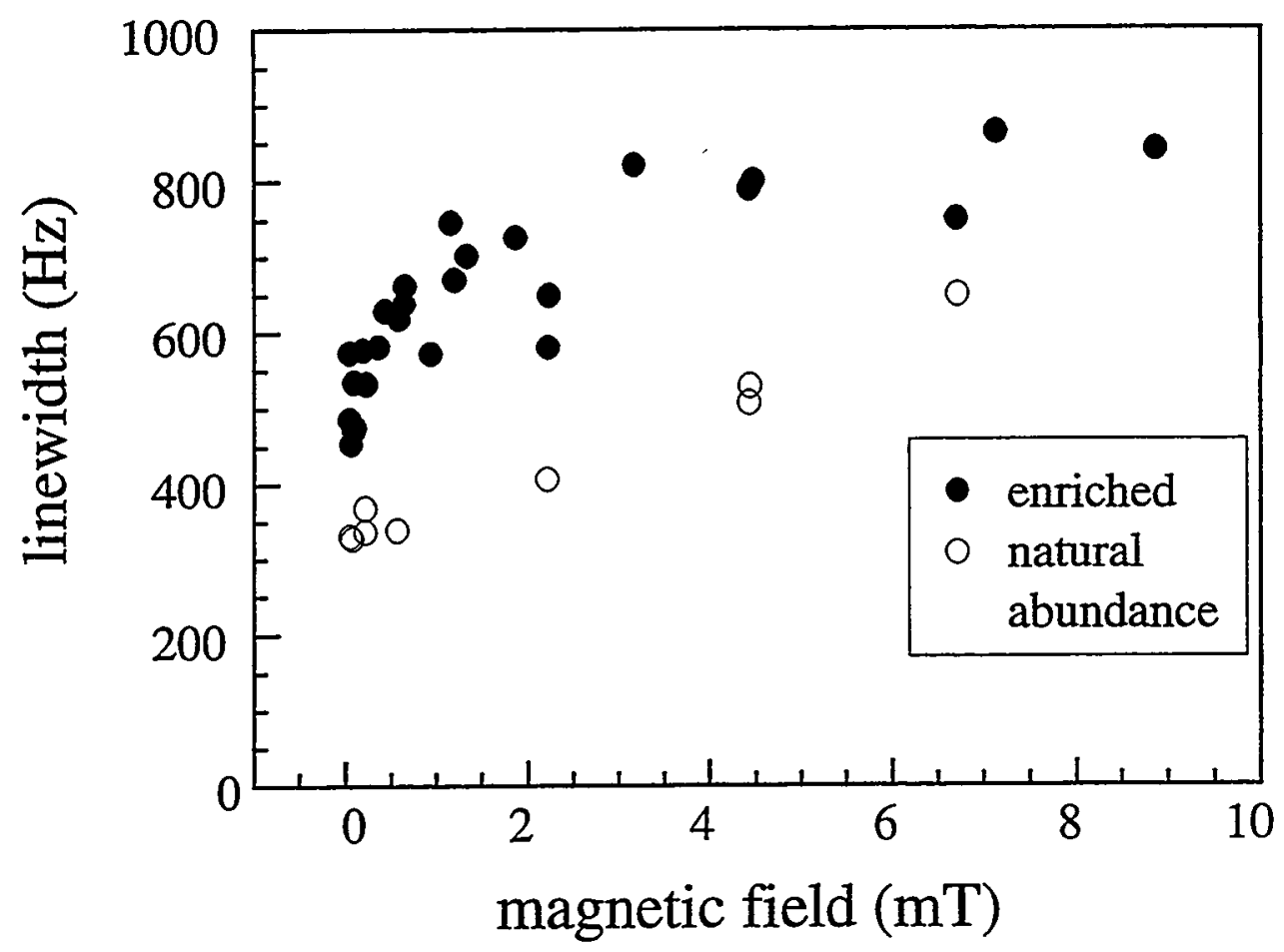

Figure 3.9: ${ }^{129} \mathrm{Xe}$ linewidth as a function of magnetic field. At low field (less than about $0.1 \mathrm{mT}$ for enriched and $0.05 \mathrm{mT}$ for naturally abundant samples), the linewidth is dominated by the dipole-dipole coupling. At the lowest field, the FWHM is $520 \pm 60 \mathrm{~Hz}$ and $340 \pm 20 \mathrm{~Hz}$ for the isotopically enriched and naturally abundant samples, respectively. The higher field inewidths are nearly linear with field and are dominated by field inhomogeneity.

were acquired with a single af excitation pulse producing a tipping angle of $5-10^{\circ}$ for Figure 3.8(a) and of close to $90^{\circ}$ for Figure 3.8(b). As shown in Figure 3.9, the linewidth increases with increasing magnetic field for both the naturally abundant samples and the isotopically enriched samples. As the magnetic field is reduced to near zero, the FWHM (full-width at half-maximum) approaches $520 \pm 60 \mathrm{~Hz}$ for the isotopically enriched samples and $340 \pm 20 \mathrm{~Hz}$ for the naturally abundant samples. Using the broadening due to dipoledipole coupling of like $\left({ }^{129} \mathrm{Xe}-{ }^{129} \mathrm{Xe}\right)$ and unlike $\left({ }^{129} \mathrm{Xe}-{ }^{131} \mathrm{Xe}\right)$ spins, we calculated the 
$616 \mathrm{~Hz}$ and $350 \mathrm{~Hz}$ linewidths for nuclear dipolar interactions for the isotopically enriched and naturally abundant samples, respectively. The increase in the linewidths at higher magnetic fields is largely due to the inhomogeneity of the solenoid, about $1 \%$. From the signal-to-noise ratio of our data, assuming a ${ }^{129} \mathrm{Xe}$ polarization of $10 \%$, we estimate that at $20 \mathrm{kHz}$ our spectrometer can detect approximately $5 \times 10^{15}{ }^{129} \mathrm{Xe}$ spins in a single shot.

The $T_{1}$ values are determined by measuring a series of ${ }^{129} \mathrm{Xe}$ signals at different times after the sample is inserted into the spectrometer. For each measurement, a small af excitation pulse is used with a tipping angle between $5^{\circ}$ and $10^{\circ}$. We determine the tipping angles in a separate experiment by applying 3-20 pulses in rapid succession and measuring the resulting decay of the signal. The tipping angle is taken into account in evaluating

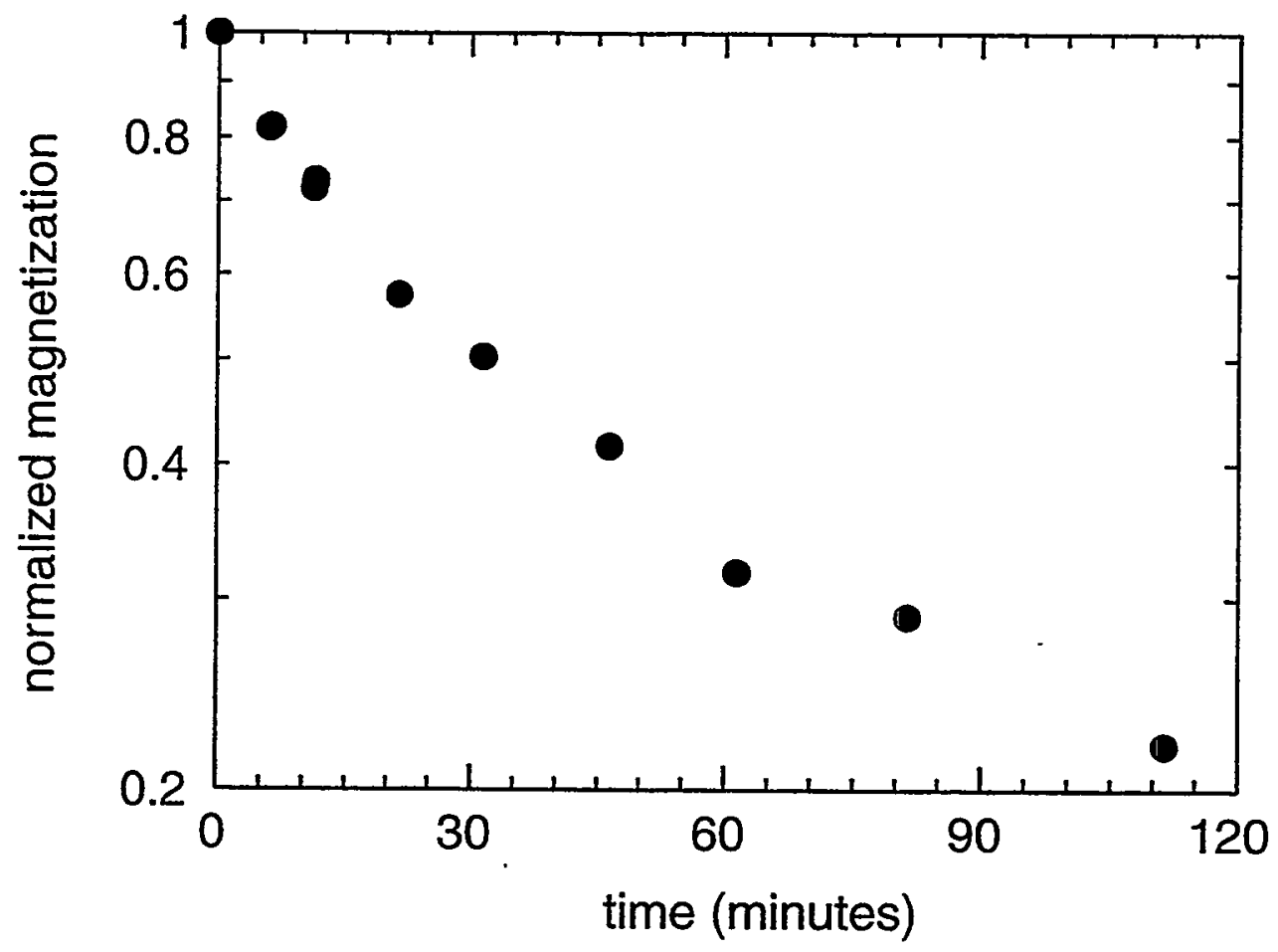

Figure 3.10: Normalized magnetization as a function of times indicating a biexponential decay. The data are given for a field of $1.3 \mathrm{mT}$. The FID of the first point can be seen in Figure 3.7. 
$T_{1}$. The decay of the xenon magnetization at most magnetic field values does not follow a single exponential function, as shown in Figure 3.10, indicating inhomogeneity in the relaxation process. We determine the $T_{1}$ from the initial decay of the signal, so that the quoted values are averaged over the distribution of the relaxation processes involved. Figure 3.11 shows $T_{1}$ vs. magnetic field for the enriched sample measured at $4.2 \mathrm{~K}$. The data of Gatzke et al.[22] over the magnetic field range 5 to $100 \mathrm{mT}$ are also indicated. We note that the value obtained at the highest field in the current work is a factor of 2 or 3 lower than in the earlier work; we return to this point later. Despite this discrepancy, we see that $T_{1}$ is strongly dependent on magnetic field above a few $\mathrm{mT}$ and relatively insensitive to magnetic field below $2 \mathrm{mT}$. As the magnetic field approaches zero, $T_{1}$ saturates at approximately $2000 \mathrm{~s}$.

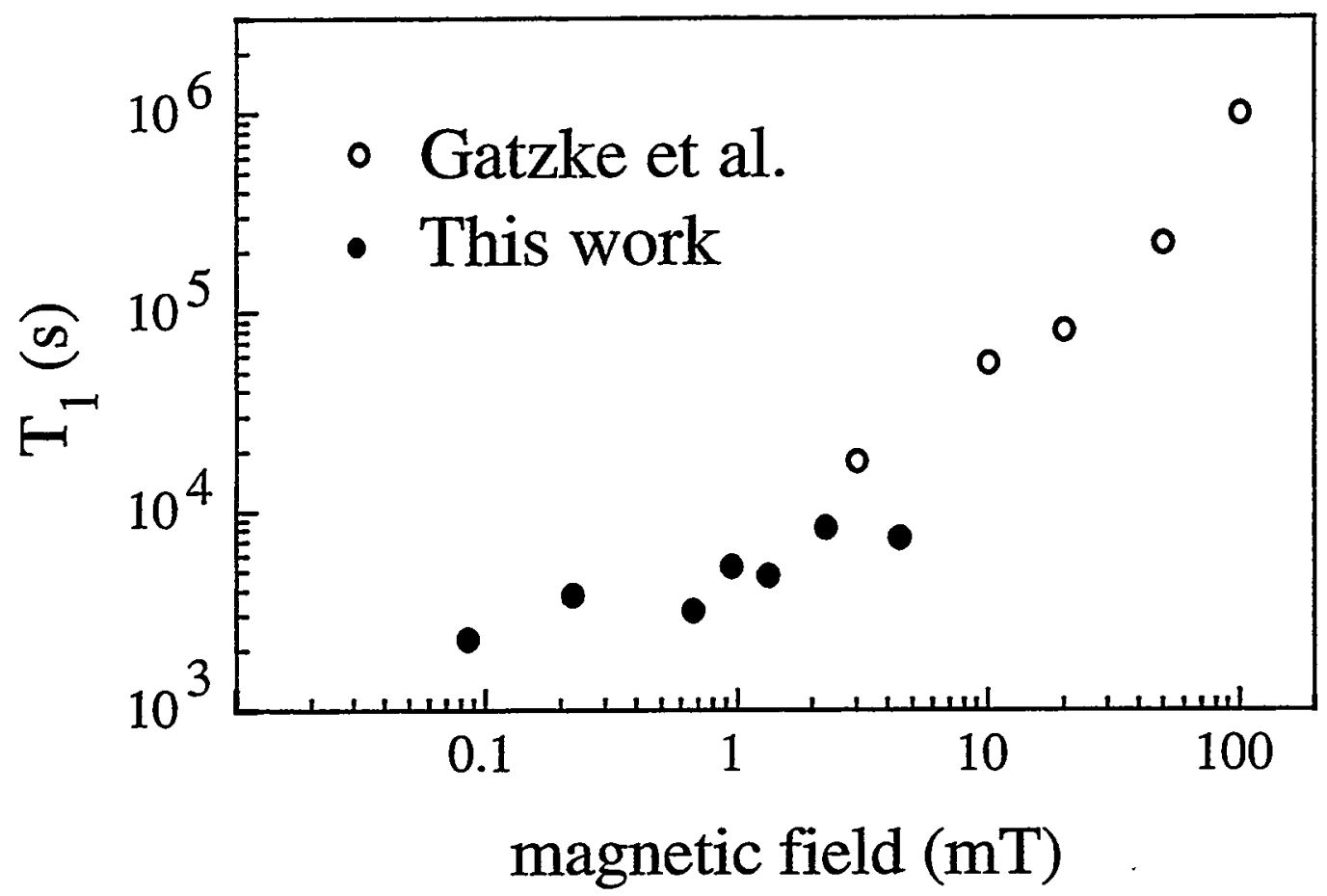

Figure 3.11: $\quad T_{1}$ for isotopically enriched (solid circles) ${ }^{129}$ Xe versus magnetic field and the data by Gatzke et al. [22] at higher magnetic field (open circles). As the magnetic field approaches zero, $T_{1}$ tends towards a value of about $2000 \mathrm{~s}$. 
We now turn to a brief discussion of the magnetic field dependence of $T_{1}$. Gatzke et al. [22] argue that at temperatures below $20 \mathrm{~K}$ the relaxation arises primarily from cross-relaxation to ${ }^{131} \mathrm{Xe}$ via the nuclear dipole interaction; because of their quadrupole moment, the ${ }^{131} \mathrm{Xe}$ nuclei couple to the lattice more effectively than ${ }^{129} \mathrm{Xe}$. The effectiveness of this cross-relaxation depends on the overlap of the ${ }^{129} \mathrm{Xe}$ and ${ }^{131} \mathrm{Xe}$ resonance lines. The fact that the measured $T_{1}$ for the ${ }^{129} \mathrm{Xe}$ nuclei is almost independent of magnetic field below $2 \mathrm{mT}$ indicates that the ${ }^{131} \mathrm{Xe}$ linewidth is very broad, about $20 \mathrm{kHz}$. This width, however, cannot be explained by dipolar broadening; the dipolar coupling of ${ }^{131} \mathrm{Xe}$ is actually weaker than that of ${ }^{129} \mathrm{Xe}$ because of the smaller gyromagnetic ratio of ${ }^{131} \mathrm{Xe}$ and the lower ${ }^{131} \mathrm{Xe}$ concentration in our sample. If the coupling of the ${ }^{131} \mathrm{Xe}$ nuclei to the electric field gradient is neglected, the ${ }^{129} \mathrm{Xe}$ and ${ }^{131} \mathrm{Xe}$ lines will not overlap until the magnetic field is decreased below $0.025 \mathrm{mT}\left(616 \mathrm{~Hz} / 2 \gamma_{129}\right)$ and $0.015 \mathrm{mT}\left(350 \mathrm{~Hz} / 2 \gamma_{129}\right)$ for the enriched and the naturally abundant samples, respectively. These fields are lower than those in any of our experiments, implying that another mechanism, namely nuclear quadrupole coupling to the lattice, is responsible for broadening the ${ }^{131} \mathrm{Xe}$ linewidth. Warren and Norberg [21] measured the linewidth of the naturally abundant ${ }^{131} \mathrm{Xe}$ at $4.2 \mathrm{~K}$ in a magnetic field of $860 \mathrm{mT}$ to be $2.4 \pm 0.2 \mathrm{kHz}$, a value still too low to account for the saturation of $T_{1}$ in our experiments. We can explain this discrepancy as follows. In the direct detection of NMR in ${ }^{131} \mathrm{Xe}$, most of the nuclei contribute to the signal and the linewidth is dominated by nuclei in the bulk rather than on the surface or at grain boundaries[22]. We believe that the opposite situation prevails in the observation of the spin-lattice relaxation 
of ${ }^{129} \mathrm{Xe}$ via cross-relaxation with ${ }^{131} \mathrm{Xe}$. In our case, the ${ }^{131} \mathrm{Xe}$ nuclei in the bulk, with a linewidth of about $2.4 \mathrm{kHz}$, do not participate in the cross-relaxation until the magnetic field is below $0.7 \mathrm{mT}$. Thus at higher fields, the observed magnetic field dependence of $T_{1}$ for the ${ }^{129} \mathrm{Xe}$ nuclei suggests that only those ${ }^{131} \mathrm{Xe}$ nuclei near grain boundaries and defects, where the higher electric field gradients produce broader resonance linewidths, contribute to the cross-relaxation. Since the grain size depends on the details of the sample preparation, which is difficult to control, the fact that relaxation is dominated by nuclear quadrupole interactions at grain boundaries may well explain why our value of $T_{1}$ at about $4.5 \mathrm{mT}$ is lower than that of Gatzke et al.[22].

\subsection{Other results and Discussion}

As discussed in the introduction, the interest in studing NMR of hyperpolarized ${ }^{129} \mathrm{Xe}$ is to use it as a polarization source for systems of low spin density. Attempts were carried out, unsuccessfully however, to cross-relax the ${ }^{129} \mathrm{Xe}$ spins to those of ${ }^{13} \mathrm{C}$ in $\mathrm{CO}$ and ${ }^{131} \mathrm{Xe}$ in naturally abundant xenon. The cross relaxation of enriched optically pumped ${ }^{129} \mathrm{Xe}$ gas to $\mathrm{CO}$ by low field thermal mixing had been done earlier by Bowers $e t$ al.[37], followed by detection of the resonance in high field NMR. In our experiment, optically pumped xenon and a sample of $\mathrm{CO}$ were allowed to mix at room temperature, Figure 3.12. Experiments were done for 1:1, 1:2,1:4,2:1, and 4:1 ratios of xenon to CO. After mixing for about 10-60 seconds, the mixed gas was frozen at the tip of the sample tube and lowered into the SQUID spectrometer. Since the gyromagnetic ratios of ${ }^{129} \mathrm{Xe}$ and ${ }^{13} \mathrm{C}$ are very similar, the field is chosen to be high enough to achieve reasonable sepa- 


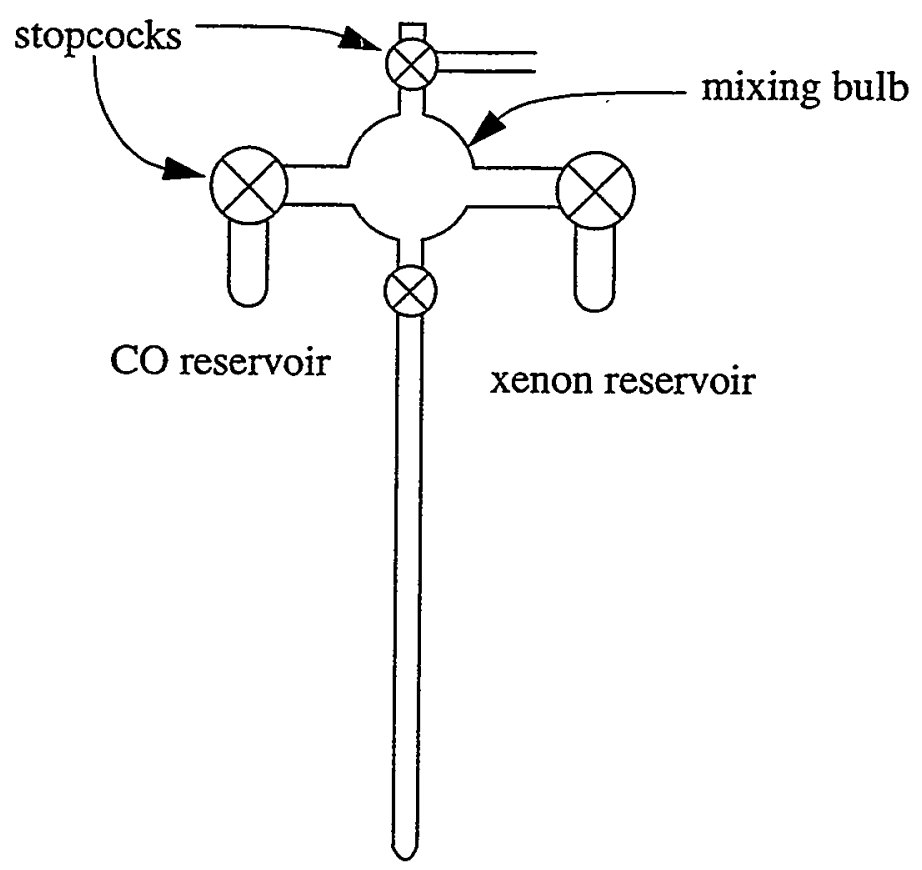

Figure 3.12: Sample tube used for xenon-CO cross-relaxation experiment. The CO gas and the xenon gas are stored in their respective reservoir, and allow to mix in a glass bulb. After some mixing time (10-60 seconds) the gas sample is frozen at the tip of the tube, which is tranferred to the SQUID spectrometer for detection.

ration of the two Larmor lines, typically about $10 \mathrm{kHz}$. The ${ }^{129} \mathrm{Xe}$ Larmor line could be clearly seen in the experiment, indicating that sufficient ${ }^{129} \mathrm{Xe}$ polarization was present; however, the ${ }^{13} \mathrm{C}$ Larmor line was never observed. One possible reason is that a Xe-CO matrix was not formed during the mixing-freezing of the sample which can result in an inefficiency of the spin transfering process. A second attempt was made to cross-relax ${ }^{129} \mathrm{Xe}$ to ${ }^{131} \mathrm{Xe}$ in a naturally abundant sample. Upon freezing, the ${ }^{129} \mathrm{Xe}$ and ${ }^{131} \mathrm{Xe}$ should form a good matrix in which cross-relaxation is efficient. However, no ${ }^{131} \mathrm{Xe}$ signal was observed. As discussed earlier in the context of ${ }^{129} \mathrm{Xe} T_{1}$ relaxation, ${ }^{131} \mathrm{Xe}$ is a quadrupole nuclei with a very large coupling. The predicted linewidth, on the order of 20 
$\mathrm{kHz}$, make the resonance very hard to observe, especially when the frequency of the ${ }^{131} \mathrm{Xe}$ Larmor line $(-1 / 2$ to $1 / 2$ transition $)$ is so low $\left(\gamma_{131}<\gamma_{129}\right)$ 


\section{References}

[1] J. Riesse, Nouv. J. Chem 10 (1985) 665.

[2] L. C. de Menorval, D. Raftery, S.-B Liu, K. Takegoshi, R. Ryoo, and A. Pines, J. Phys. Chem. 94 (1990) 27.

[3] A. Kastler, J. Phys. Radium 11 (1950) 255.

[4] T. R. Carver, Science 141 (1963) 599.

[5] W. Harper, Rev. Mod. Phys. 44 (1972) 169.

[6] M.S. Albert, G.D. Cates, B. Driehuys, W. Happer, B. Saam, C.S. Springer Jr., A. Wishnia, Nature 370 (1994) 199.

[7] H. Middleton, R.D. Black, B. Saam, G.D. Cates, G.P. Cofer, R. Guenther, W. Happer, L.W. Hedlund, G.A. Johnson, Kl Juvan, J. Swartz, J. Magnetic Resonance. Med. 33 (1995) 271.

[8] M. Leduc, E. Otten, La Recherche 287 (1996) 41.

[9] K. Sakai, A.M. Milek, E. Oteiza, R.L. Walsworth, D. Balamore, F. Jolesz, M.S. Albert, J. Mag. Res. B 111 (1996) 300.

[10] R.R. Swanson, M. Rosen, J.W. Stokes, P. Downing, Anaesth. Intensive Care 1996 (preprint)

[11] G. Navon, Y.-Q. Song, T. Room, S. Appelt, R.E. Taylor, A. Pines, Science 271 (1996) 1848.

[12] A. Bifone, Y.-Q. Song, R. Seydoux, R.E. Taylor, B.M. Goodson, T. Pietrab, T. Budinger, G. Navon, A. Pines, Proc. Natl. Acad. Sci. USA (1996)

[13] T. Room, S. Appelt, R. Seydoux, E.L. Hahn, A. Pines, Phys. Rev. B (submitted)

[14] D. Raftery, H. Long, T. Meersman, P.J. Grandinetti, L. Reven, A. Pines, Phys. Rev. Lett. 66 (1991) 584. 
[15] C.R. Bowers, H.W. Long, T. Pietrab, H.C. Gaede, A. Pines, Chem. Phys. Lett. 205 (1993) 168.

[16] H.C. Gaede, Y.-Q Song, R.E. Taylor, E.J. Munson, J.A. Reimer, A. Pines, Appl. Mag. Res. 8 (1995) 373.

[17] K.W. Zilm, D.M. Grant, J. Am. Chem. Soc. 103 (1981) 2913.

[18] Rtreever, H.Y. Carr, Phys. Rev. 121 (1961) 20.

[19] K. Oikarinen, J. Jokisaari, Appl. Mag. Res. 8 (19995) 587.

[20] W.M. Yen, R.E. Norberg, Phys. Rev. 131 (1963) 269.

[21] W.W. Warren Jr., R.E. Norberg, Phys. Rev. 154 (1967) 277.

[22] M. Gatzke, G.D. Cates, B. Driehuys, D. Fox, W. Happer, B. Saam, Phys. Rev. Lett. 70 (1993) 690.

[23] Dinh M. TonThat, M. Ziegeweid, Y.Q- Song, E.J. Munson, S. Appelt, A. Pines, John Clarke, Chem. Phys. Lett. 272 (1997) 245.

[24] F. Bloch, W.W. Hansen, and M. Packard, Phys. Rev. 70 (1946) 474.

[25] W.G. Proctor and F.C. Yu, Phys. Rev. 81 (1951) 550

[26] R.R. Emst, Chimia 29 (1975) 179.

[27] A. Kastler, J. Phys. Radium 11 (1950) 255

[28] A. Kastler, Science 158 (1967) 214.

[29] T.G. Walker and W. Happer, Rev. Mod. Phys. 69 (1997) 629

[30] H.G. Dehmelt, Phys. Rev. 109 (1958) 381.

[31] W. Happer, E. Miron, S. Shaefer, D. Schreiber, W.A.V. Wijngaarden, X. Zeng, Phys. Rev. A 29 (1984) 3092.

[32] X. Zeng, Z. Wu, T. Call, E. Miron, D. Schreiber, W. Happer, Phys. Rev. A 31 (1985) 260.

[33] C.C. Bouchiat, M. A. Bouchiat, L. C. Pottier, Phys. Rev. 181 (1969) 144. 
[34] M. A. Bouchiat, J. Brossel, L.C. Pottier, J. Chem. Phys. 56 (1972) 3703.

[35] B. C. Grover, Phys. Rev. Lett. 40 (1978) 390.

[36] C.H. Volk, T.M. Kwon, J.G. Mark, Y.B. Kim, J.C. Woo, Phys. Rev. Lett. 44 (1980) 136.

[37] C.R. Bowers, H.W. Long, T. Pietrass, H. Gaede, A. Pines, Chem. Phys. Lett. 205 (1993) 168. 


\section{Chapter 4}

\section{SQUID MRI of Laser-polarized ${ }^{3} \mathrm{He}$ and ${ }^{129} \mathrm{Xe}$ at $4.2 \mathrm{~K}$}

\subsection{Introduction}

Unaided, the human eye can image objects as small as $0.1 \mathrm{~mm}$, and because of strong absorption and reflection of light by most materials the eye can view only the surface of materials. The discovery of X-rays by Roentgen in the early 20 th century enabled us to see inside materials and to observe the structural features within objects for the first time. In the early 70s, absorption images from a thin axial layer were taken with an X-ray computed tomography (CT) scanner[1]. A key element for the development of these scanners, commonly known as CAT scanners, is the advancement and availability of highspeed digital computers used to reconstruct the images. Still, the resolution of the images obtained by CAT scanners is limited, due to the beam collimation process. In 1972, Lauterbur and Mansfield and Grannell demonstrated experimentally the use of NMR as a tool for macroscopic imaging and the field of magnetic resonance imaging (MRN) was 
born[2,3]. Aside from the fact that MRI applies no radiation to the patient, the advantages of MRI over X-ray CT include a higher theoretical resolution and the capability of slice selection of an image at any angle. MRI has quickly advanced to the point that it has become a routine tool in medical diagnostics[4-6] and materials characterization[7]. To achieve high sensitivity and resolution, current MRI instruments require a magnetic field of a several tesla, giving a typical proton Larmor frequency of $100 \mathrm{MHz}$. Superconducting magnets are typically used to produce such field which are $\sim 10^{4}$ times the earth's field. Such magnets are not only expensive to buy and to maintain, but can pose hazardous conditions to persons wearing pacemakers or having metal tranplants or prosthetics. Serious injuries related to the use of MRI in hospital have been documented. One example is a case in which a patient had a serious respiratory problem during an MRI examination. As the attending nurse was trying to bring him an oxygen tank, the magnet sucked the tank into its core pinning the patient down. The only way to get the patient out was to quench the magnet. In view of the potential harzard of operating a large field, the question to ask is whether or not MRI can be done in a much lower field, such as the field of $\sim 10 \mathrm{mT}$ present in a magnet of a normal household refrigerator. Performing MRI in such fields, or even lower fields, not only eliminates the above-mentioned concerns, but also presents new potential applications for MRI, including real-time MRI during surgerical procedures.

As discussed earlier, performing NMR and MRI experiments in low field results in small, "hard to see" signals with the Faraday induction method. The use of a SQUIDbased spectrometer alleviates part of this problem, and SQUTDs have been used to detect proton magnetic resonance images in fields down to $B_{0}=9 \mathrm{mT}$, where the NMR frequency is $\sim 400 \mathrm{kHz}[8,9]$. One attractive MRI application is to image porous materials and sur- 
faces. One way to image the porous materials is to fill the sample with high sensitivity contrast agents and thereby obtain a "negative MRI image" of the void space. Typical contrast agents are solvents containing high concentrations of protons to give large signals. However, because of their inertness, noble gases are ideal non-intrusive contrast agents for cases in which these solvents might react or bind to the materials of interest[10,11]. With the development of the use of optically pumped noble gases as contrast agents for MRI of surfaces[12-14] and of void spaces[15,16], a new scheme is to use a SQUID for imaging these gases at low fields. In this chapter, I will present the combination of optical pumping and SQUID detection that dramatically increases the sensitivity of noble gas MRI at low frequencies[17], opening the way to the practical implementation of MRI without the need for high magnetic fields.

\subsection{MRI Theory}

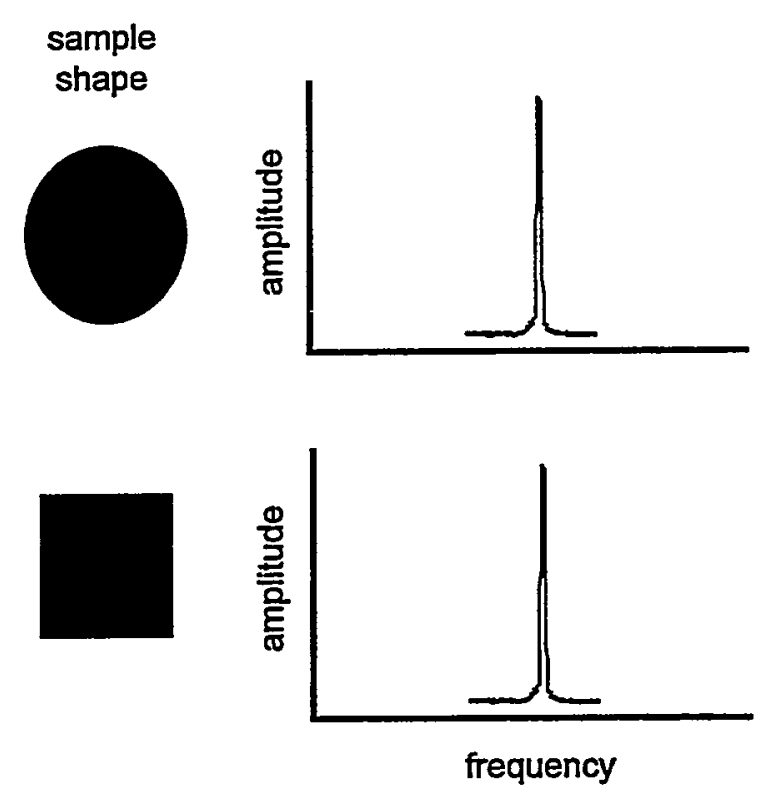

Figure 4.1: In the presence of a homogeneous static magnetic field, spherical and cubic objects will give the same sharp resonance line. 


\subsubsection{Basic Theory}

In this section I will briefly discuss MRI theory; a good source for this can be found in Callaghan's Principles of Nuclear Magnetic Resonance Microscopy[7]. As discussed in Section 3.2, in the presence of a homogeneous magnetic field the energy levels of a spin $1 / 2$ system split into two, giving a sharp resonance line that is independent of the shape of the sample as shown in Figure 4.1. The crucial idea, first proposed by Lauterbur [2], is to use a linear field gradient $G$ to disperse the NMR resonance frequency of the sample and to encode the spatial information within the acquired spectrum. The point can be easily illustrated for the 1-D MRI image of a 2-D object as shown in Figure 4.2.
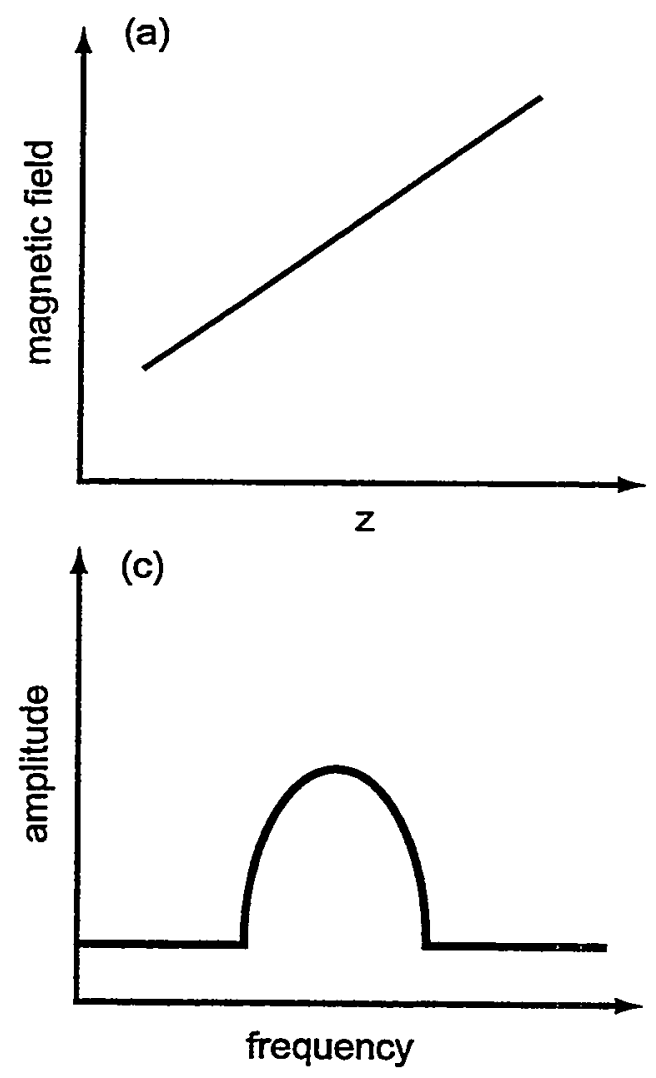

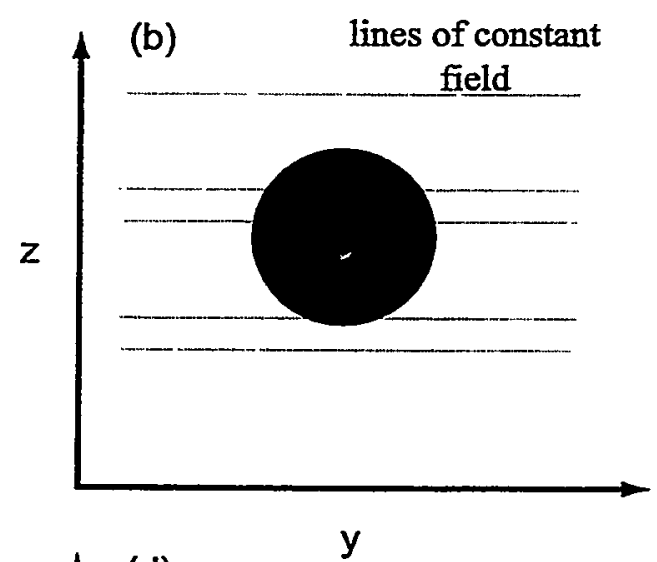

(d)

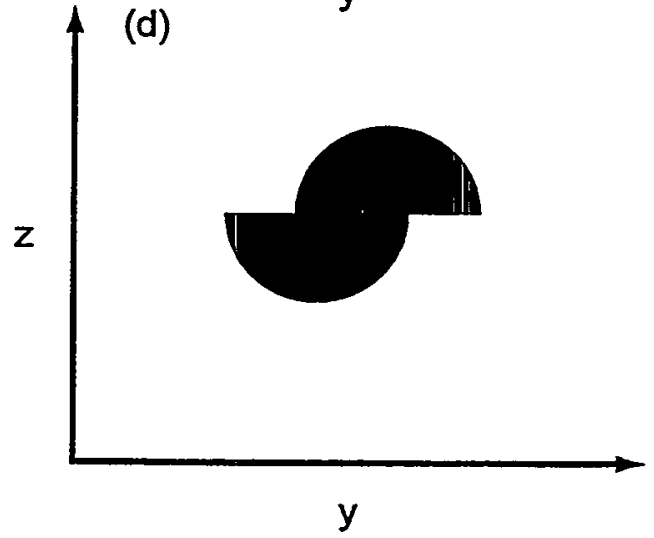

Figure 4.2: (a) The profile of the field with a gradient $G_{z}$ along the $z$ direction. (b) The sample in this field will give a spectrum shown in (c), where frequency is proportional to $\gamma G_{z}$. Sample of shape in (d) will also give the same spectrum shown in (c) under the influence of the same field profile (a). 
Assuming the FID is taken with a constant gradient $G_{z}$ along a $z$ direction having a field profile as shown in Figure 4.2 (a), regions of constant magnetic field will map on to a specific frequency of an amplitude that is dependent on the projection of the spin density of the sample revolving at that frequency. For a circular sample, the acquired spectrum would look like Figure 4.2 (c). Since this is a projection of a 2-D image onto a 1-D frequency space, some information will be lost; thus, the 1-D MRI spectrum will be the same for the images shown in Figure 4.2(b) and Figure 4.2(d). A more general mathematical formulation can be given as follows. In the presence of a static field $B_{0} \hat{z}$ and a field gradient $G=\bar{\nabla} B_{z}$ the spin precesses at the resonance frequency

$$
\omega(\boldsymbol{r})=\gamma\left(B_{0}+\boldsymbol{G} \cdot \boldsymbol{r}\right),
$$

which is dependent on its location within the sample. The signal $d S$ obtained from a volume $d r$, shown in Figure 4.3, is

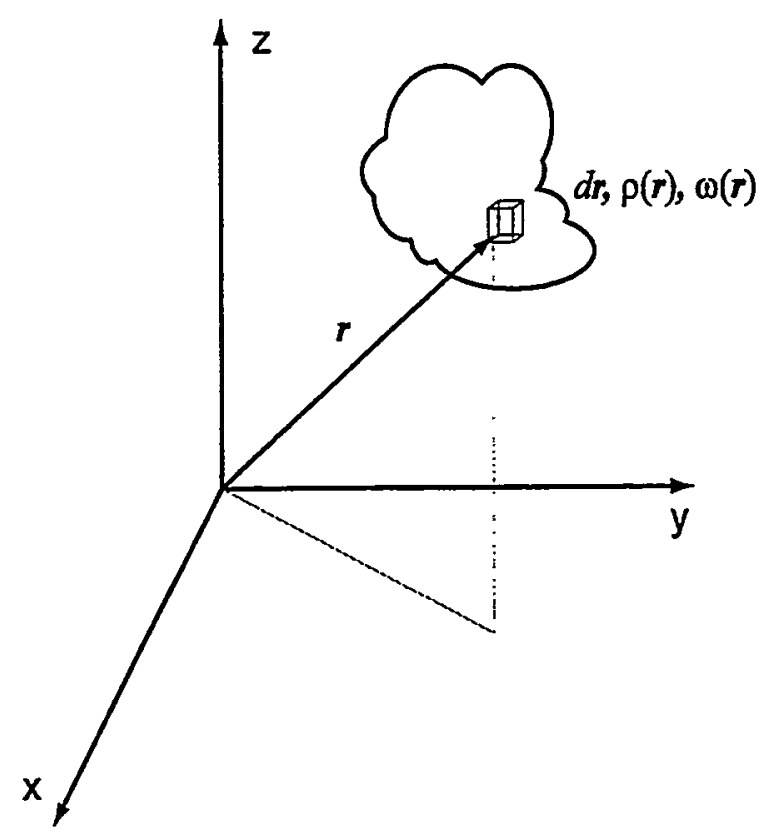

Figure 4.3: The signal emitted from a small volume $d r$ is proportional to the number of spins $\rho(r) d r$ times the phase $\mathrm{e}^{i \omega(r) t}$ at that location. 


$$
d S \propto \rho(r) e^{i \omega(r) t} d r
$$

For simplicity, ignoring the constant of proportionality and choosing a reference frequency to be $\gamma B_{0}$, the so-called 'on resonance' condition, the signal becomes

$$
S(\boldsymbol{G}, t)=\iiint \rho(r) e^{i \gamma \boldsymbol{G} \cdot \boldsymbol{r} t} d \boldsymbol{r}
$$

By introducing a reciprocal vector $k(k=\gamma G t / 2 \pi)$, we find

$$
S(k)=\iiint \rho(r) e^{i 2 \pi k \cdot r} d r
$$

and

$$
\rho(\boldsymbol{r})=\iiint S(k) e^{-i 2 \pi k \cdot r} d k
$$

In performing an MRI experiment, we wish to obtain $\rho(r)$. Equation 4.1 tells us that $\rho(r)$ is just the Fourier transform of the signal $S\left(k_{x}, k_{y} k_{z}\right)$ that we measured in the time domain. For the case in Figure 4.2, the signal is

$$
S\left(k_{z}\right)=\int_{-\infty-\infty}^{\infty} \int_{-\infty}^{\infty} \rho(y, z) e^{i 2 \pi k_{z} z} d y d z
$$

and the Fourier Transform of the signal is

$$
F\left(S\left(k_{z}\right)\right)=\rho(z)=\int_{-\infty}^{\infty} \rho(y, z) d y,
$$

which is the spin density $\rho(y, z)$ projected on the $z$-axis (the axis defined by the gradient direction).

\subsubsection{Two-Dimensional Fourier Imaging}

It is of important to see how a typical MRI image is taken. By applying a gradient, 


\section{$G_{y}$}
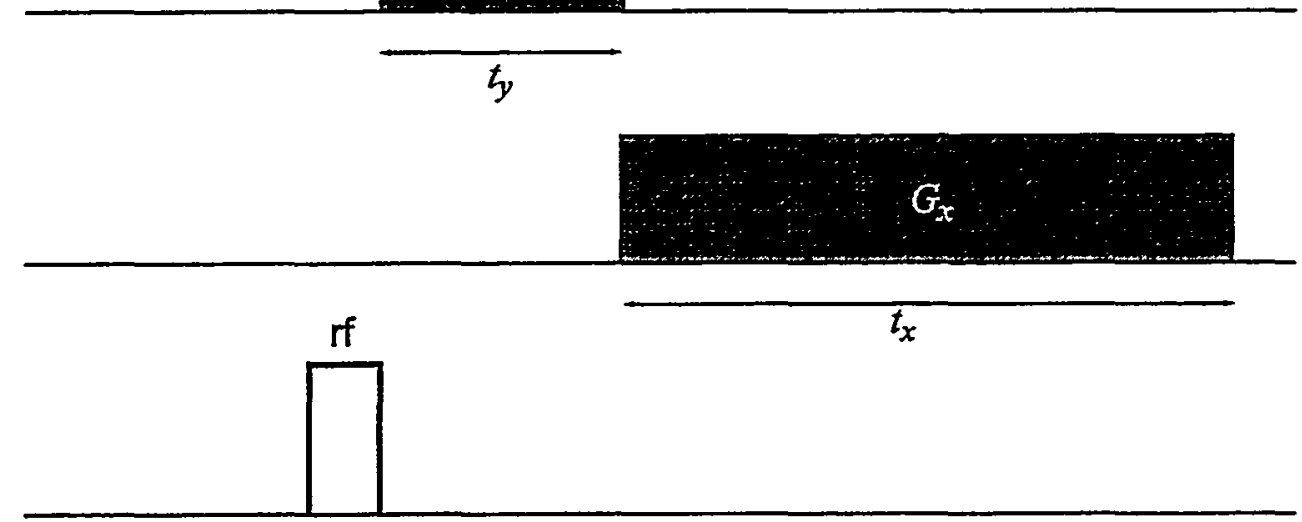

Figure 4.4: In the 2-D version of the Fourier Imaging experiment, a slice is selected out by applying a selective pulse in the presence of a $z$ gradient $G_{z}$. The sampling in $k_{x}-k_{y}$ is done by acquiring the FIDs in the presence of $G_{x}$ after the spins are allowed to evolve in the presence of $G_{y}$ for a time $t_{y}$

say $G_{z}$, and pulsing softly on the sample, a certain slice parallel to the $x y$-plane can be selected. Sampling of the $k_{x}$ and $k_{y}$ is then required for the reconstruction of this $\mathrm{x}$-y image slice. When one FID is taken, signal points obtained are along a single line in this $k_{x}-k_{y}$ space; thus for a 2-D reconstruction, a 'design' set of FIDs is required to map out the $k$ space. An example of this is the Fourier Imaging technique shown in Figure 4.4. After a slice is selected, a gradient $G_{y}$ is applied in the $y$ direction to encode the phase of the signal, while $G_{x}$ is on during the acquisition of the FIDs. The signal obtained in this Fourier Imaging scheme is

$$
S\left(k_{x}, k_{y}\right) \rightarrow S\left(t_{x}, t_{y}\right)=S(0,0) e^{i \gamma\left(G_{y} t_{y}+G_{x} t_{x}\right)},
$$

where $k_{y}$ is usually sampled by fixing $t_{y}$ and varying $G_{y}$ for each FID taken. The desired 


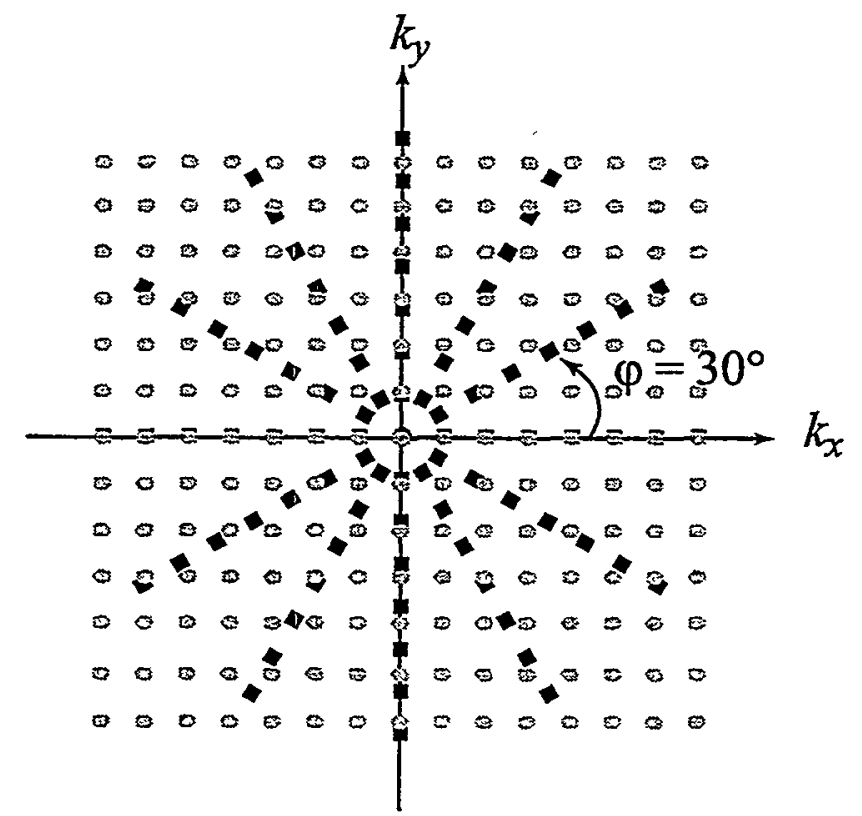

Figure 4.5: A polar raster (black square) is acquired when the spectra are observed in the presence of gradients along different angle $\varphi$. Signals are then interpolated to a Cartesean raster (grey circle), and Fourier transformed to yield the image.

image is obtained by taking the 2-D Fourier transform of the acquired data.

\subsubsection{Projection Reconstruction}

Now imagine, instead, the FID is acquired for an arbitrary gradient in this $k_{x}-k_{y}$ space, and after each FID is taken, the sample (or the gradient coil) is rotated by an angle $\varphi$. In doing so, we will obtain a sampling that is radial in $k$-space $\mathrm{S}(k, \varphi)$, Figure 4.5 . The image can be obtained by performing the following integral

$$
\rho(r, \varphi)=\iint S(k, \varphi) e^{-i 2 \pi k \cdot r} k d k d \varphi
$$

However, unlike the sampling in Cartesian coordinates, this integral is no longer a Fourier transform and the inverse problem is somewhat complicated. For the reconstruction of the image, sampling of $\mathrm{S}(k, \varphi)$ is first converted to $S\left(k_{x}, k_{y}\right)$ by a 4-point interpolation process 
and the image $\rho(x, y)$ is obtained by performing a Fourier transform on this $S\left(k_{x}, k_{y}\right)$.

\subsection{Experimental Setup}

The preparation of high ${ }^{129} \mathrm{Xe}$ and ${ }^{3} \mathrm{He}$ spin polarizations is accomplished by spin exchange of the noble gas with optically pumped $\mathrm{Rb}$ metal vapor, discussed in Section 3.3. The ${ }^{3} \mathrm{He}$ sample is made by sealing a cylindrical pumping cell $15 \mathrm{~cm}$ long and $0.6 \mathrm{~cm}$ in internal diameter containing 1 bar of ${ }^{3} \mathrm{He}, 13$ mbar of $\mathrm{N}_{2}$ gas, and a few milligrams of $\mathrm{Rb}$ metal[18]. The ${ }^{129} \mathrm{Xe}$ sample, on the other hand, is a sealed cylindrical cell $1.5 \mathrm{~cm}$ long

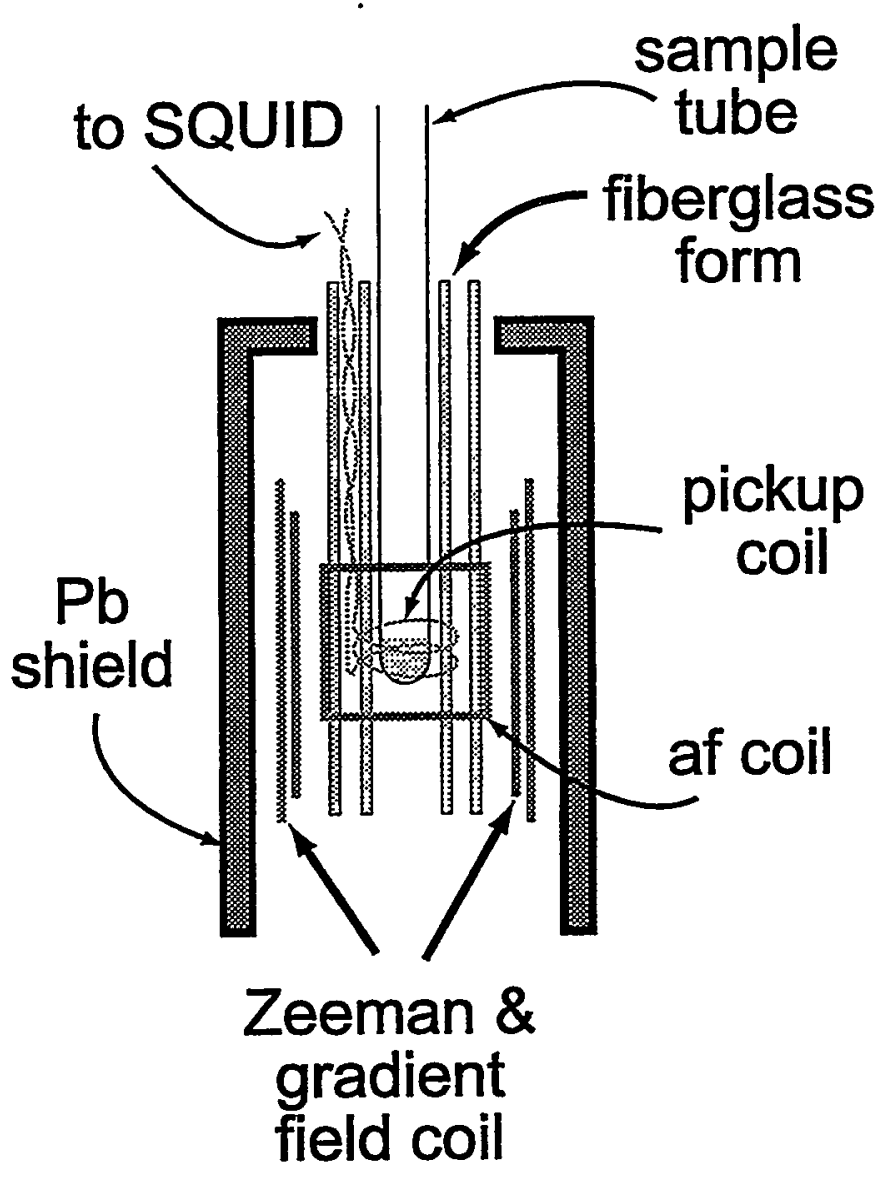

Figure 4.6: Configuration of the probe assembly used to obtain ${ }^{3} \mathrm{He}$ and 129 magnetic resonance images at a magnetic field $1 \mathrm{mT}$, showing the relative placement of the coils, sample, and $\mathrm{Pb}$ shield. 
and $0.48 \mathrm{~cm}$ in internal diameter containing 2 bar of ${ }^{129} \mathrm{Xe}, 20$ mbar of $\mathrm{N}_{2}$ gas, and a few milligrams of $\mathrm{Rb}$ metal. The optical pumping proceeds by heating the ${ }^{129} \mathrm{Xe}$ cell to $400 \mathrm{~K}$ for 5 minutes and the ${ }^{3} \mathrm{He}$ cell to $470 \mathrm{~K}$ for 0.5 to 4 hours while irradiating the contents with $1 \mathrm{~W}$ of $794.74 \mathrm{~nm}$ laser light directed along the magnetic field of $50 \mathrm{mT}$. This process orients the $\mathrm{Rb}$ electron spins parallel to the magnetic field resulting in a polarization of the ${ }^{129} \mathrm{Xe}$ or ${ }^{3} \mathrm{He}$ nuclear spins to a few percent. The probe assembly used in this study is shown in Figure 4.6. The sample tube, immersed in liquid helium, is placed at the center of a pair of coils, each with a rectangular shape $65 \times 24 \mathrm{~mm}$ and wound with 30 turns of superconducting $\mathrm{NbTi}$ wire that supplies the static magnetic field $B_{0}$. A second pair of coils, each with a rectangular shape $60 \times 20 \mathrm{~mm}$ and with 5 turns of $\mathrm{Nb}$ wire, has a common axis with the $B_{0}$-field coils and provides the magnetic field gradient $G$. The field profiles of the static and gradient coils are shown in Figure 4.7. The magnetic signal produced by
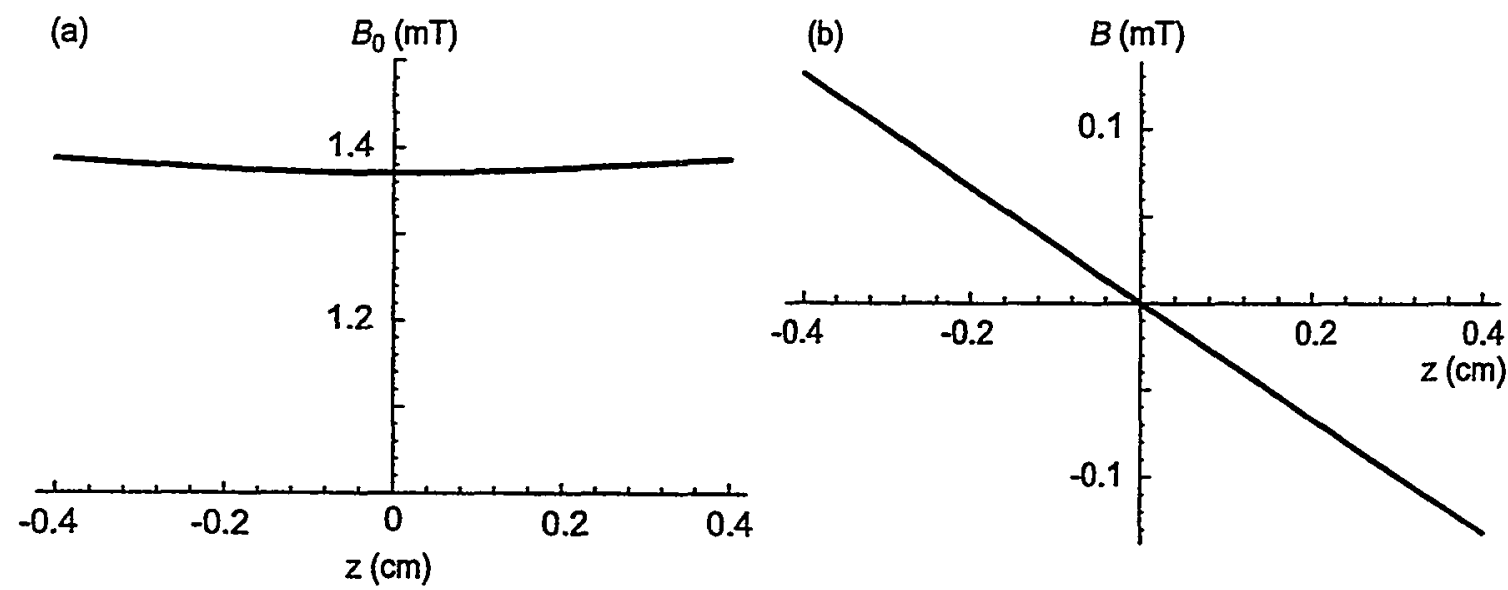

Figure 4.7: $\quad$ (a) The field profile of the $B_{0}$ coil, calculated for a current of $1 \mathrm{~A}$ through the coil. The average field is about $1.38 \mathrm{mT}$ with an inhomogeneity of $1.5 \%$ within the region of the sample. (b) The field profile of the gradient coil, also calculated for a current of $1 \mathrm{~A}$. The gradient is about $0.033 \mathrm{~T} / \mathrm{m}$. Under the influence of a $\mathrm{Pb}$ shield these values are reduced considerably. 
the precessing nuclei is coupled into a two-turn, $\mathrm{Nb}$-wire solenoid with its axis along the axis of the cylindrical sample. The audio-frequency (af) transmitter coil is orthogonal to the pickup coil and static field coils, and consists of two planar 20-turn coils positioned $15.6 \mathrm{~mm}$ apart. The static magnetic field and gradient coils are orthogonal to both the af coil and the pickup coil and are operated in a persistent-current mode using two separate superconducting switches. In this way a magnetic field up to $1.5 \mathrm{mT}$ and field gradient up to $0.1 \mathrm{~T} / \mathrm{m}$ can be applied perpendicularly to the symmetry axis of the cylindrical sample. Because the input circuit has a response that extends to zero frequency, to exclude ambient magnetic noise it is necessary to surround it with the superconducting $\mathrm{Pb}$ shield shown in

Figure 4.6. This shield reduces the static and gradient fields by a factor of about one-half from the values calculated from the geometry of the coils driven by a current ranging from 0.5-3 A. To calibrate the static field and gradient coils and to test the ability of our system to obtain an image we first performed a series of experiments on ${ }^{3} \mathrm{He}$ gas.

\subsection{Result and Discussion}

\subsubsection{1-D MRI of ${ }^{3} \mathrm{He}$}

One-dimensional images of ${ }^{3} \mathrm{He}$ gas were obtained using a two-pulse spin echo sequence [19]. A typical echo response is shown in Figure 4.8 for a $6 \mathrm{~mm}$ diameter tube of laser-polarized ${ }^{3} \mathrm{He}$ gas ( $10 \%$ spin polarization) at a pressure of $\sim 1$ bar. The two af pulses (each consisting of one cycle at a frequency $\omega_{\mathrm{af}} / 2 \pi=17 \mathrm{kHz}$ with an amplitude of 0.09 $\mathrm{mT}$ ) were separated by $0.66 \mathrm{~ms}$, leading to a refocusing of the magnetization at $1.32 \mathrm{~ms}$. Apodization of the echo signal with a hyperbolic secant centered at $1.32 \mathrm{~ms}$ and having a 


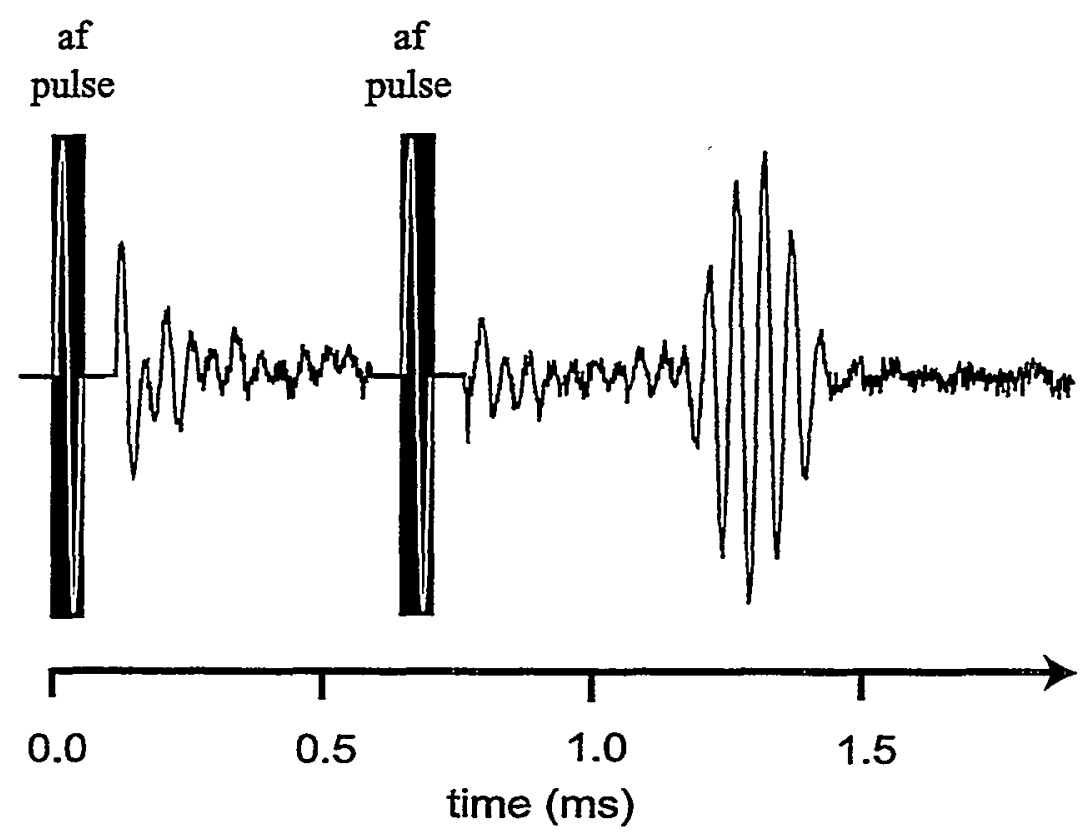

Figure 4.8: Spin echo obtained for ${ }^{3} \mathrm{He}$ at $0.56 \mathrm{mT}$ in gradient of $0.054 \mathrm{~T} / \mathrm{m}$. Each excitation pulse (denoted as black box in the figure) consisted of one cycle at 17 $\mathrm{kHz}$ with an amplitude of $0.09 \mathrm{mT}$ giving a tipping angle of $61^{\circ}$. The two pulses were separated by $0.66 \mathrm{~ms}$. The receiver-gating time following each pulse was 61 $\mu$.

width of $1.5 \mathrm{~ms}$, followed by Fourier transformation, yields the spectrum shown in Figure 4.9(a). The applied magnetic field gradient inferred from the width of the spectrum is $G=0.054 \mathrm{~T} / \mathrm{m}$ while the static field calculated from the center of gravity of the line and the gyromagnetic ratio for ${ }^{3} \mathrm{He}$ is $B_{0}=0.56 \mathrm{mT}$. Reducing the gradient to zero yields the spectrum in Figure 4.9(c), with a center frequency determined by the current of $1 \mathrm{~A}$ applied to the static field coil giving $B_{0}=0.56 \mathrm{mT}$. The observed linewidth in spectrum Figure $4.9(\mathrm{c})$ is most likely due to the inhomogeneity of $B_{0}$, although the relaxation times $T_{1}$ and $T_{2}$ of ${ }^{3} \mathrm{He}$ gas at $4.2 \mathrm{~K}$ and $0.56 \mathrm{mT}$ have not been quantified.

The shapes of the ${ }^{3} \mathrm{He}$ images in Figure 4.9(a,b) are consistent with the circular distribution function expected for a linear gradient perpendicular to the symmetry axis of a 

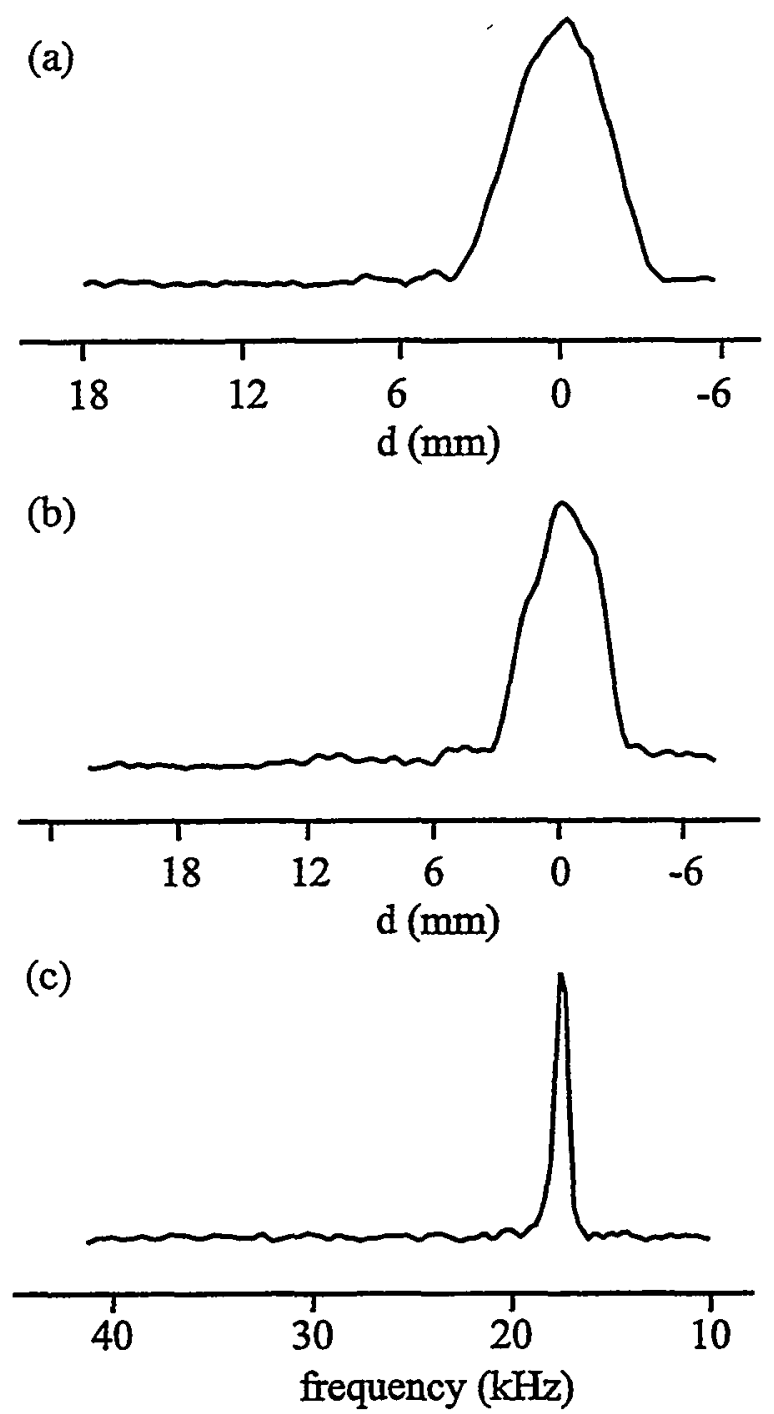

Figure 4.9: $\quad{ }^{3} \mathrm{He}$ spectra as a function of magnetic field gradient.The abscissa in (c) provides the same frequency scale for all the three figures. The abscissa in (a) and (b) provide the spatial scales for these two figures.(a) Fourier transform of the signal following the second af pulse in Figure 4.8 with $B_{0}=0.56 \mathrm{mT}$ and $G=0.054 \mathrm{~T} / \mathrm{m}$; these values were inferred from the gyromagnetic ratio of ${ }^{3} \mathrm{He}$, the center and width of the line (a) and the known diameter of the sample tube. The static magnetic field coils were driven with $1 \mathrm{~A}$ and the gradient coils with $3 \mathrm{~A} . B_{0}=0.56 \mathrm{mT}$ remains constant when the current applied to the gradient coils is decreased to $2 \mathrm{~A}$ in (b) and to zero in (c). The gradient in (b) is $G=0.035 \mathrm{~T} / \mathrm{m}$ calculated from the width of the line and the known diameter of the sample tube. The resolution in the ${ }^{3} \mathrm{He}$ gas images is (a) $500 \mu \mathrm{m}$ and (b) $800 \mu \mathrm{m}$. 
cylindrical sample, Figure 4.2(c). From the experimentally obtained linewidth in Figure4.9(c) and the known shape and inner diameter of the ${ }^{3} \mathrm{He}$ sample container we obtain an estimate for the resolution of $500 \mu \mathrm{m}$ in Figure 4.9(a) and $800 \mu \mathrm{m}$ in Figure 4.9(b).

The spectra do not show the diffusive edge enhancement[20,21] reported recently by Saam et al.[22] in a room temperature ${ }^{3} \mathrm{He}$ sample at a higher field of $3.1 \mathrm{mT}$ and a smaller gradient of $0.001 \mathrm{~T} / \mathrm{m}$. Spin diffusion causes attenuation of the signal, and signal enhancement near regions of less diffusion such as boundary and constriction can distort the reconstructed image of the sample[21]. The absence of this enhancement is presumably due to the low temperature used in the present experiments, and the projected cylindrical symmetry of the sample tube which can conceal lineshape features near the edge of the image.

\subsubsection{2-D MRI of Solid ${ }^{129} \mathrm{Xe}$}

Because of the long ${ }^{3} \mathrm{He}$ sample, ${ }^{3} \mathrm{He}$ polarization is quickly depolarized by diffusion to regions of zero field. Thus performing a 2-D MRI on our ${ }^{3} \mathrm{He}$ sample was not possible. Knowing the $T_{1}$ of solid ${ }^{129} \mathrm{Xe}$ in the presence of a small field to be extremely long at $4.2 \mathrm{~K}$ (Chapter 3 ), we used polarized solid ${ }^{129} \mathrm{Xe}$ to demonstrate the feasibility of lowfield, SQUID-detected MRI. A two-dimensional image was obtained by sequentially rotating the cylindrical sample tube in the gradient field applied perpendicularly to its symmetry axis as discussed in Section 4.2.3. In this way the distribution of the spins in the $x y$-plane could be reconstructed from the radial sampling. The raw 2-D image shown in Figure 4.10(a) is constructed from 12 sample orientations with 30 free induction decay 


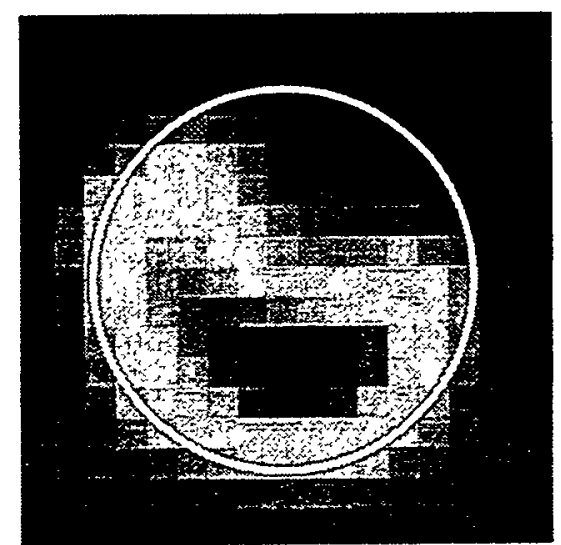

$1 \mathrm{~mm}$ (a)

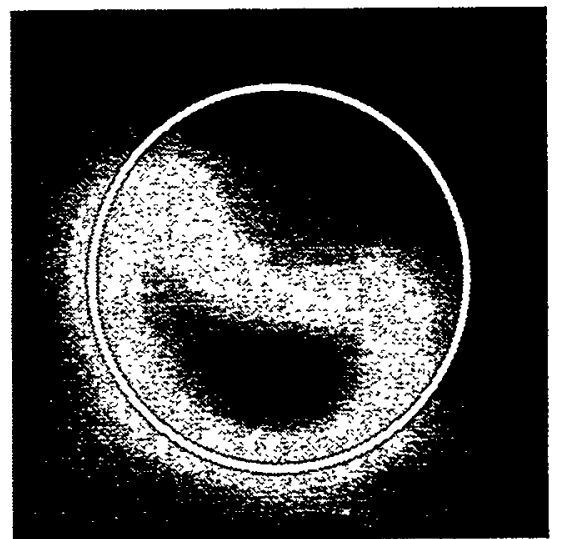

(b)

Figure 4.10: (a) Raw ${ }^{129} \mathrm{Xe}$ images collected at $1 \mathrm{mT}$ and $0.054 \mathrm{~T} / \mathrm{m}$. Currents of $2 \mathrm{~A}$ and $3 \mathrm{~A}$ applied to the $B_{0}$-coils and the gradient coils, respectively. The image results from 12 angular steps of $30^{\circ}$ each, with 30 free induction decay points at each angle. The 2-D image has a resolution of $920 \mu \mathrm{m}$. Smoothing this image with a linear four-point spline interpolation routine produces (b). The white line included in both images corresponds to the $4.8 \mathrm{~mm}$ inner diameter of the tube containing the ${ }^{129} \mathrm{Xe}$.

points per orientation. The 12 separate data sets were collected on the same xenon sample at $B_{0}=1 \mathrm{mT}$ and $G=0.054 \mathrm{~T} / \mathrm{m}$ using af pulses having a tipping angle of $10^{\circ}$. The white line in Figure 4.10 represents the $0.48 \mathrm{~cm}$ inner-diameter of the pyrex tube containing the solid xenon. Linear four-point spline interpolation of these data produces the image shown in Figure $4.10(b)$. Evidently the xenon was frozen to one side of the sample tube. From the calibrated gradients and the known $600 \mathrm{~Hz}$ linewidth of solid ${ }^{129} \mathrm{Xe}$ in the absence of a gradient [23] we estimate a resolution of $920 \mu \mathrm{m}$ for the 2-D image. This value is only a factor of five greater than that observed for solid, laser-polarized ${ }^{129} \mathrm{Xe}$ obtained at high magnetic field (4.2 T) by conventional NMR detection, $100 \mathrm{~mm}$ [24]. However, better res- 
olution is expected for a homogeneous field and a higher field gradient.

\subsection{Future Work}

Even though the current SQUID spectrometer produced excellent images at low fields, the results obtained so far are only for samples at $4.2 \mathrm{~K}$, the temperature of the cryostat. However, it should be possile to perform room-tempature, low-field MRI with SQUID arrays[25] similar to that used for in vitro and in vivo biomagnetic measurements of magnetic fields from nerves, skeleton muscle, and cardiac tissue or with a modified high $T_{c}$ scanning SQUID microscope used in our group to perform nondestructive evaluation of materials and to study magnetostatic bacteria[26]. Efforts are underway in our laboratory to design a high- $\mathrm{T}_{\mathrm{c}}$ SQUID NMR and NQR spectrometer, similar to the high- $\mathrm{T}_{\mathrm{c}}$ scanning SQUID microscopes, specifically to perform low field NMR and MRI studies of samples at room temperature. The SQUID will be operated at $77 \mathrm{~K}$ separated by $\leqslant 100 \mu \mathrm{m}$ from the room-temperature sample by a window. From preliminary calculations, the expected MRI signal for optically pumped noble gas is expected to be comparable to that obtained in this work. 


\section{References}

[1] G.N. Hounsfield, Br. J. Radiol. 46 (1972) 1016.

[2] P.C. Lauterbur, Nature 242 (1973) 190.

[3] P. Mansfield and P.K. Grannell, J. Phys. C 6 (1973) L422.

[4] P.C. Lauterbur, in NMR in Biology and Medicine, S. Chien and C. Ho, Eds. (Raven Press, New York, 1986).

[5] P. Mansfield and P.G. Morris, NMR Imaging in Biomedicine (Acedamic Press, New York, 1982).

[6] D.G. Gadian, Nuclear Magnetic Resonance and its Application to Living Systems (University Press, Oxford, 1982).

[7] P.T. Callaghan, Principles of Magnetic Resonance Microscopy (Clarendon Press, Oxford, 1991).

[8] S. Kumar, W.F. Avrin, and B.R. Whitecotton, IEEE Trans. on Magn. 32 (1996) 5261.

[9] H.C. Seton, D.M. Bussell, J.M.S. Hutchison, and D.J. Lurie, IEEE Trans. on Appl. Supercond. 5 (1995) 3218.

[10] J.A. Ripmeester, D.W. Davidson, Bull. Magn. Res. 2 (1981) 139.

[11] T. Ito, J. Fraissard in 5th International Conference on Zeolites (Heyden, Namples) (1980) 150.

[12] M.S. Albert, G.D. Cates, B. Driehuys, and W. Happer, Nature 370 (1994) 199.

[13] H. Middleton, R.D. Black, B. Saam, G.D. Cates, G.P. Cofer, R. Gunther, W. Happer, L.W. Hedlund, G.A. Johnson, K. Juvan, J. Swartz, Mag. Reson. Med. 33 (1995) 271.

[14] S.D. Swanson, M.S. Rosen, B.W. Agranoff, K.P. Coulter, R.C. Welsh, and T.E. Chupp, paper presented at the 38th Experimental Nuclear Magnetic Resonance 
Conference, Orlando, FL, 1997.

[15] G. Navon, Y. -Q. Song, T. Room, S. Appelt, R. E. Taylor, A. Pines, Science 271 (1996) 1848.

[16] M.S. Albert, G.D. Cates, B. Driehuys, W. Happer, et. al., Nature 370 (1994) 199.

[17] M.P. Augustine, D.M. TonThat, A. Wong-Foy, J.L. Yarger, M. Tomaselli, J. Clarke, and A. Pines, App. Phys. Lett. (submitted).

[18] M.P. Augustine and K.W. Zilm, Mol. Phys. 89 (1996) 737.

[19] E. L. Hahn, Phys. Rev 80 (1950) 580.

[20] P.T. Callaghan, A. Coy, L. Ford, and C. Rofe, J. Magn. Reson. A 101 (1993) 347.

[21] T.M. De Swiet, J. Magn. Reson. B 109 (1995) 12.

[22] B. Samm, N. Drukker, and W. Happer, Chem. Phys. Lett. 263 (1996) 481.

[23] Dinh. M. TonThat, M. Ziegeweid, Y.-Q. Song, E.J. Munson, S. Appelt, A. Pines, and J. Clarke, Chem. Phys. Lett. 272 (1997).

[24] Y.-Q. Song, R.E. Taylor, and A. Pines, Solid State Nucl. Magn. Reson., in press

[25] G.L. Romani, C. Del Gratta, and V. Pizzella, in SQUID Sensors: Fundamentals, Fabrication and Applications, edited by $\mathrm{H}$. Weinstock (Kluwer Academic, Dordrecht, the Netherlands, 1996), p. 445.

[26] T.S. Lee, Y.R. Chemla, E. Dantsker, J. Clarke, IEEE Trans. App. Supp. 7 (1997) 3147. 


\section{Chapter 5}

\section{SQUID-Detected NQR of ${ }^{27} \mathrm{Al}$ and ${ }^{2} \mathrm{D}$}

\subsection{Introduction}

Quadrupole nuclei with spin $\gg 1 / 2$ represent more than 70 of the elements in the periodic table[1]. Nuclear electric quadrupole resonance (NQR) arises from the interaction of these nuclei with the local electric field gradient (EFG) as shown in Figure 5.1(a). The interaction lifts the degeneracy of the spin energy levels. By studying the resonance between the energy levels, the local EFG can be extracted to provide information about the local bonding configuration, the symmetry of the molecular bonding, the symmetry of the lattice, and the effect of defects on the environment of the nucleus[2]. Traditional NQR experiments are limited to frequencies above $5 \mathrm{MHz}$ where the resonance frequency is large enough to induce appreciable signal in a tuned Faraday coil. Low-resonant NQR (0 - 5MHz) techniques included methods such as field cycling, nuclear double resonance, and high-field NMR[2]. 


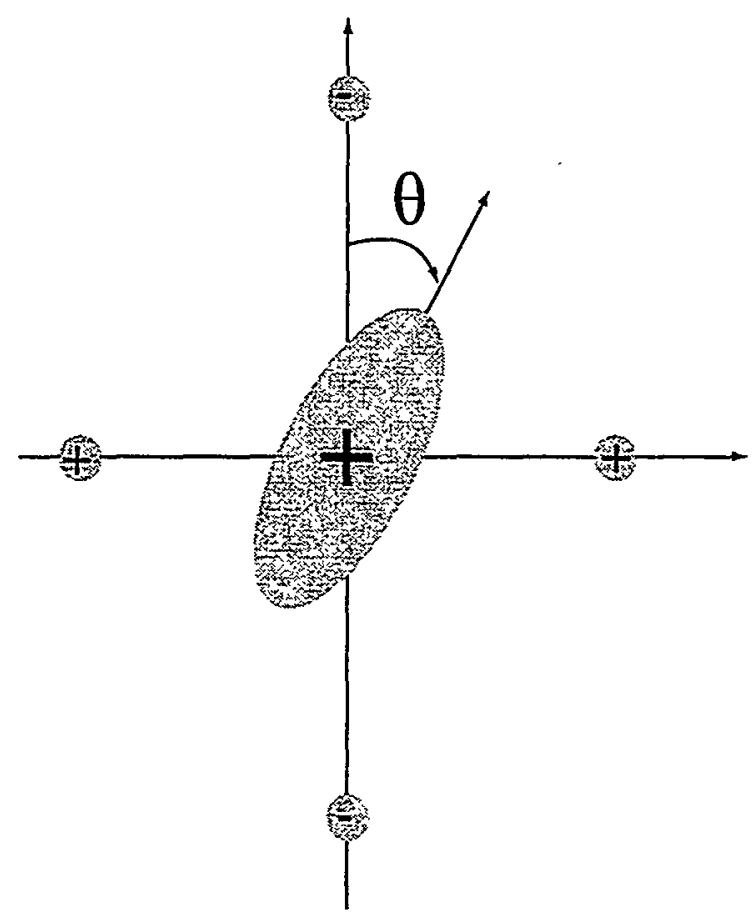

Figure 5.1: A nonspherical nucleus in the electric field gradient of the 4 charges has a preferred orientation. When $\theta$ is zero, the configuration is most favorable. The gradient is usually due to to the unsymmetrical distribution of bonding electrons surrounding the nucles. Thus by studying this gradient the symmetry of bonding and lattice can be extracted. The EFG, however, can also arise from the local defects.

Field cycling has been used to acquire high resolution NQR spectra. In this technique, the sample is allowed to equilibrate in a region of high field $B_{0}$ and then transported to a region of zero field where the spins evolve for a time $t$ before the sample is returned to $B_{0}$ where the amplitude of the evolved magnetization is detected. The FID is then reconstructed by plotting this magnetization versus the evolved time $t$; the process can be very time consuming in many cases. Also, the $T_{1}$ relaxation time of the sample must be sufficiently long to prevent the polarization relaxing away during the transition of the sample from a region of high field to a region of low field. Nuclear double resonance is a way to detect the NQR of one nuclear spin species by means of level crossing to different nuclei 
species, such as from ${ }^{14} \mathrm{~N}$ to ${ }^{1} \mathrm{H}$. In the presence of a Zeeman field matching the ${ }^{1} \mathrm{H}$ Larmor line to one of the ${ }^{14} \mathrm{~N}$ lines, the populations of the upper and lower levels of the two spin species become equal by mutual "spin-flips". The NQR resonance is indirectly monitored by measuring the change in the proton NMR signal in high field as a function of the frequency of an applied irradiating field in a lower field. Since ${ }^{14} \mathrm{~N}$ FD can not be obtained with this technique, relaxation information can not be precisely extracted. NQR information can also be extracted by performing high field NMR, as long as the NQR is just a perturbation on the Zeeman interaction. Because of the low bandwidth of typical NMR setups, this particular technique is limited to single crystal samples or samples with nuclei having a very small NQR interaction, like that of deuterium ${ }^{2} \mathrm{D}$. In polycrystalline or powder samples, the random orientations of the spins with respect to the Zeeman field give rise to a broad spectrum which can conceal structural information. If the spectrum is very broad, only part of this broad spectrum is acquired in a single experiment, thus making the analysis difficult.

An alternative scheme is to detect low resonant frequency $N Q R$ signals in zero field, the "pure NQR" case, directly with a superconducting coil coupled to a dc SQUID. In the frequency range of interest, the SQUID-based amplifier has a noise temperature much lower than that of a semiconductor amplifier. An earlier SQUID pulsed spectrometer, operating up to frequency of $200 \mathrm{kHz}$, was successfully used to detected ${ }^{14} \mathrm{~N} \mathrm{NQR}$ of $\mathrm{NH}_{4} \mathrm{ClO}_{4}$ and ${ }^{2} \mathrm{D} \mathrm{NQR}$ of perdeuterated toluene and picoline[3]. In this chapter, I will show an application of the new pulsed SQUID system to detect ${ }^{27} \mathrm{Al}$ in $\mathrm{Al}_{2} \mathrm{O}_{3}\left[\mathrm{Cr}^{+3}\right]$ at $360 \mathrm{kHz}$ and $720 \mathrm{kHz}$, and then present the higher resolution data obtained on the ${ }^{2} \mathrm{D}$ 
NQR of perdeuterated toluene and picoline. The current SQUID spectrometer is a good candidate for studying NQR of nuclei such as ${ }^{5} \mathrm{Be},{ }^{11} \mathrm{~B},{ }^{14} \mathrm{~N}$, and ${ }^{27} \mathrm{Al}$ where the NQR frequencies range between $10 \mathrm{kHz}$ up to $6 \mathrm{MHz}$.

\subsection{NQR Theory}

A simple version of $\mathrm{NQR}$ theory will be presented here; a more detail discussion can be found in T.P. Das and E.L. Hahn's Nuclear Quadrupole Resonance Spectroscopy[4]. The pure quadrupolar Hamiltonian in its principal axis system (PAS) can be written as

$$
H_{Q}=\frac{e^{2} q Q}{4 I(2 I-1)}\left[3 I_{z}^{2}-I^{2}+\frac{\eta}{2}\left(I_{+}^{2}+I_{-}^{2}\right)\right]
$$

where $e^{2} q Q$ is the quadrupole coupling constant, describing the strength of the quadrupolar interaction, and $I_{z}, I_{+}$, and $I_{-}$are the components of the nuclear spin angular momentum in the PAS. The asymmetry parameter of the electric field gradient $\eta$ is defined as

$$
\eta=\frac{V_{x x}-V_{y y}}{V_{z z}}
$$

where

$$
V_{x_{i} x_{j}}=\frac{\partial^{2} V}{\partial x_{i} \partial x_{j}}
$$

and $V$ is the electrostatic potential at the nucleus due to the surrounding charges. Because of the Laplace equation

$$
\nabla^{2} V=V_{x x}+V_{y y}+V_{z z}=0
$$

and the assumed condition in the PAS 


$$
\left|V_{x x}\right| \leq\left|V_{y y}\right| \leq\left|V_{z z}\right|
$$

$\eta$ can only vary between 0 and 1 . The case $\eta=0$ corresponds to the axial symmetry about $z$, and $\eta=1$ corresponds to $V_{x x}=0$ where the electric field gradient is limited to the $z y-$ plane.

\subsection{1 $\eta=0$}

For the case $\eta=0$, Eqn (5.1) can be rewritten as

$$
H_{Q}=\frac{e^{2} q Q}{4 I(2 I-1)}\left[3 I_{z}^{2}-I^{2}\right] .
$$

Since the Hamiltonian commutes with $\boldsymbol{I}_{z}$, the magnetic quantum number of $\boldsymbol{I}_{z}, m$, is also a good quantum number for the Hamiltonian. If $m$ and $n$ represent the states of the Hamiltonian $H_{Q}$, then the matrix element is

$$
\left\langle m\left|H_{Q}\right| n\right\rangle=\frac{e^{2} q Q}{4 I(2 I-1)}\left[3 m^{2}-I(I+1)\right] \delta_{m n},
$$

where $\delta_{m n}$ is the Kronecker delta function. As shown in Figure 5.2, the energy are doubly degenerate in $m$ since the states $|m\rangle$ and $|-m\rangle$ have the same energy, except when $m=0$. In the presence of a if magnetic field of frequency $\omega$ in the $x$ or $y$ direction, there is a transition between the spin states different by 1 and this transition rate is maximum when $\omega$ satisfies

$$
\omega=\omega_{m} \equiv \frac{E_{m+1}-E_{m}}{\hbar} .
$$

From Eq. (5.2),

$$
\omega_{m}=\frac{3 e^{2} q Q}{4 I(2 I-1) \bar{\hbar}}[2 m+1] .
$$



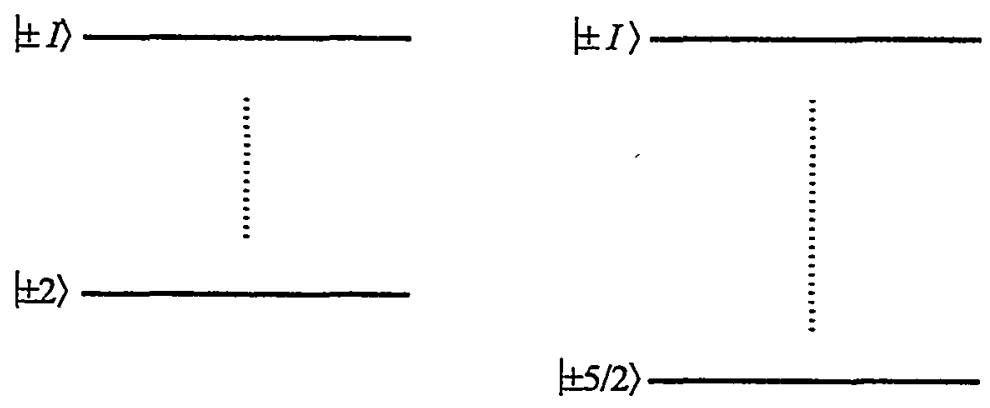

$\lfloor 1\rangle$

$43 / 2\rangle$

$|0\rangle$

$\pm 1 / 2\rangle$

Figure 5.2: The energy-level diagrams of a nuclei of spin $I$ for the case of axial symmetry about $z$. The states are labeled by $|m\rangle$ where $m$ ranges from $-I$ to $+I$. The energy levels are doubly degenerate, except for the energy level of $m=0$. Because of selection rule $\Delta m= \pm 1$, there will be $I-1 / 2$ transitions for nuclei of half-integral spin and $I$ transitions for nuclei of integral spins.

Thus, for a nuclei of spin $I$, there will be $I-1 / 2$ transitions for half-integral spin and $I$ transitions for integral spins. The quadrupolar coupling, hence the strength of the quadrupolar interaction, can be obtained very accurately by measuring the resonance frequencies, if $m$ is known. However, when two or more frequencies are observed the value can be uniquely determined. For $I=1$ or $I=3 / 2$, the problem of determining the quadrupolar coupling does not arise since only one resonance is possible, where $m=0$ and $1 / 2$, respectively.

\subsection{2 $\eta \neq 0$}

For $\eta \neq 0$, the analysis of the pure NQR spectra can be very complicated. Now $m$ is no longer a good quantum number for the Hamiltonian. If $m$ and $n$ are the magnetic quantum number of $I_{z}$, the matrix element of the Hamiltonian can be calculated in the basis of $I_{z}$ to be

$$
\left\langle m\left|H_{Q}\right| n\right\rangle=A\left\{\left[3 m^{2}-I(I+1)\right] \delta_{m n}+\frac{\eta}{2}\left[\delta_{m} \delta_{n+2} C_{n}^{+} C_{n+1}^{+}+\delta_{m} \delta_{n+2} C_{n}^{+} C_{n+1}^{+}\right]\right\}
$$


where

$$
A=\frac{e^{2} q Q}{4 I(2 I-1)}
$$

and

$$
C_{m}^{ \pm}=\sqrt{(I \mp m)(I \pm m \pm 1)} .
$$

Of course, the solution to the Hamiltonian can be solved by diagonalizing the matrix to find the eigenstates and eigenvalues. Suffice to say, often no analytic expression for the eigenstates and eigenvalues of the Hamiltonian can be written down, except in the simplest cases $I=1$ and $3 / 2$.

For the case $I=1$ which is of considerable interest because it applies to ${ }^{2} \mathrm{D}$ and ${ }^{14} \mathrm{~N}$, the solution can be solved exactly. The Hamiltonian can be expressed as

$$
H_{Q}=A\left[\begin{array}{ccc}
1 & 0 & \eta \\
0 & -2 & 0 \\
\eta & 0 & 1
\end{array}\right]
$$

Solving for the Hamiltonian, we find that the three eigenstates are

$$
\begin{aligned}
& |+\rangle=\frac{(|1\rangle+|-1\rangle)}{\sqrt{2}} \\
& |+\rangle=\frac{(|1\rangle-|-1\rangle)}{\sqrt{2}} \\
& |0\rangle=|0\rangle,
\end{aligned}
$$

where $|1\rangle,|-1\rangle$, and $|0\rangle$ are the eigenstates of $I_{z}$. The eigenvalues of these states, respectively, are

$$
\begin{aligned}
& E_{+}=A(1+\eta), \\
& E_{-}=A(1-\eta),
\end{aligned}
$$




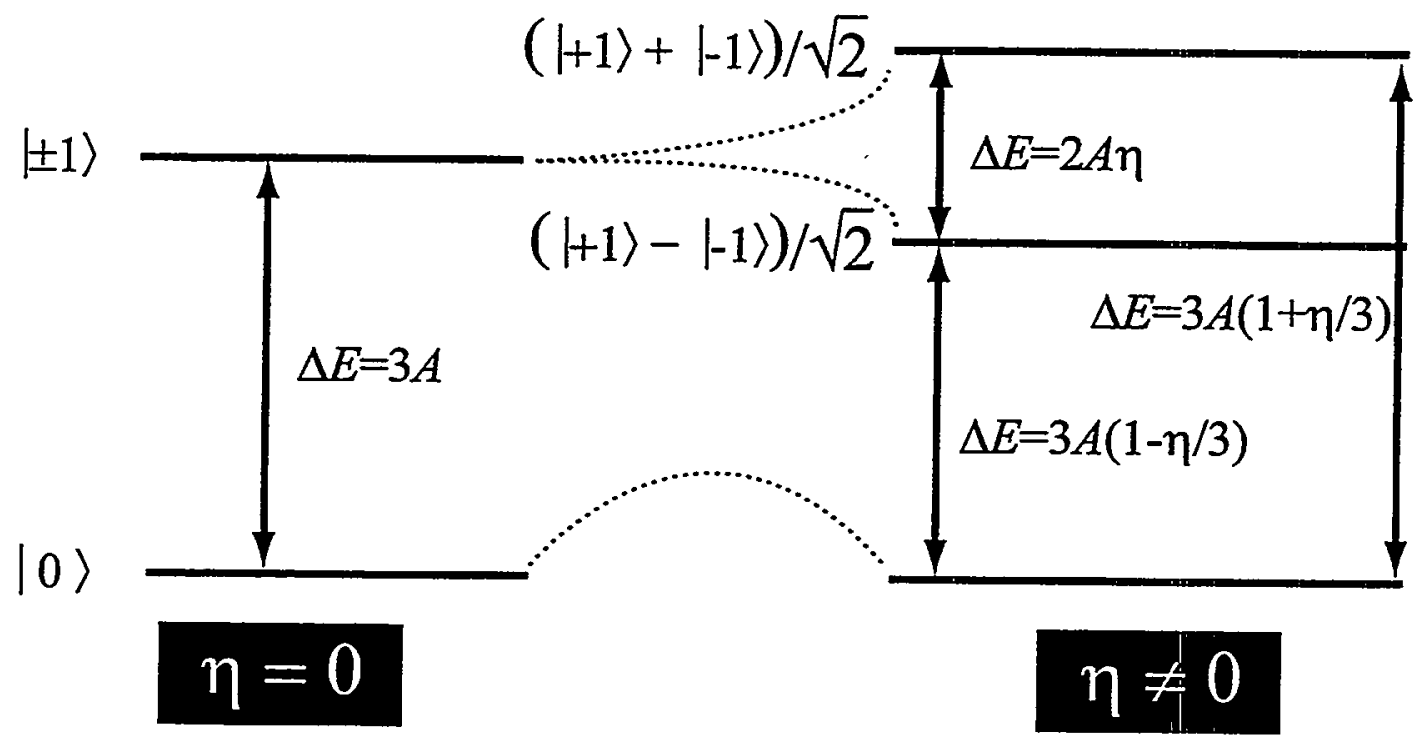

Figure 5.3: The energy levels for $I=1$. On the left is the case of $\eta=0$, and on the right is the case of $\eta \neq 0$. The presence of the asymmetry in the $x y$ plane mixes the states $|+1\rangle$ and $|-1\rangle$ to form two new non-degenerate states. Three transtions are allowed with the following transition energies: $2 A \eta, 3 A(1-\eta / 3)$, and $3 A(1+\eta / 3)$.

and

$$
E_{0}=-2 A \text {. }
$$

Transitions between all three states of the Hamiltonian are possible with the transition energies of $2 A \eta, 3 A(1-\eta / 3)$, and $3 A(1+\eta / 3)$ as shown in Figure 5.3. Of course only two of the resonant frequencies are required for the determination of the quadrupolar constant and the asymmetry parameter.

Another case where is the Hamiltonian can be exactly solved is $I=3 / 2$. The Hamiltonian is

$$
H_{Q}=A\left[\begin{array}{cccc}
3 & 0 & \sqrt{3} \eta & 0 \\
0 & -3 & 0 & \sqrt{3} \eta \\
\sqrt{3} \eta & 0 & -3 & 0 \\
0 & \sqrt{3} \eta & 0 & 3
\end{array}\right]
$$




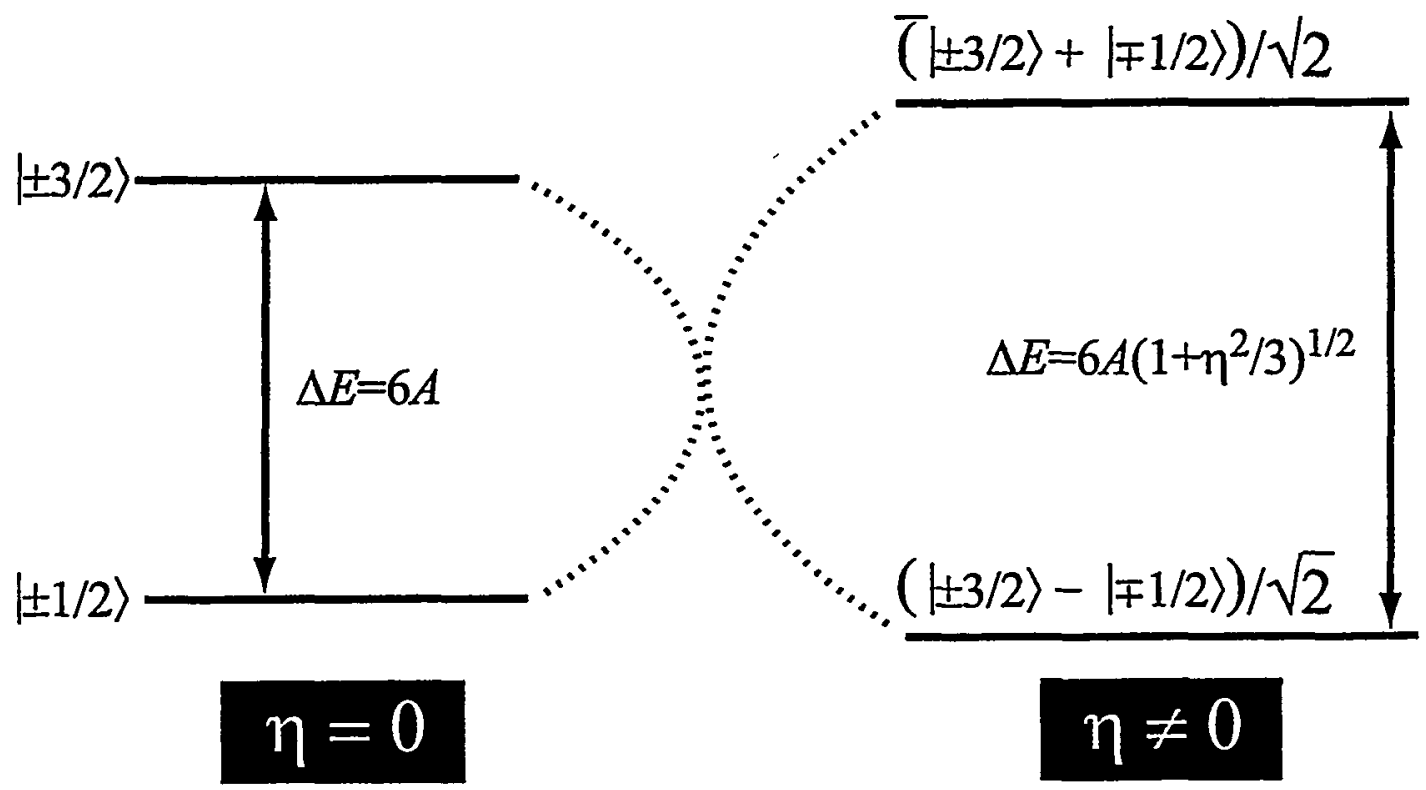

Figure 5.4: The energy levels for $I=3 / 2$. On the left is the case of $\eta=0$, and on the right is the case of $\eta \neq 0$. The presence of the asymmetry in the $x y$ plane mixes the states $\mid 3 / 2>$ and $\mid-1 / 2>$ and $\mid-3 / 2>$ and $\mid 1 / 2>$ to form four new states. However, each energy is still two-fold degeneracy and thus there is only one transition having transition energy of $6 A\left(1+\eta^{2} / 3\right)^{1 / 2}$.

As shown in Figure 5.4, the solution gives two energy levels of $\pm 3 A\left(1+\eta^{2} / 3\right)^{1 / 2}$, each with a degeneracy of two. There is only one transtion in this case, so that it is impossible to determine both $A$ and $\eta$ with "pure NQR". However, a small field can be applied to remove the degeneracy, allowing for the determination of both $A$ and $\eta$.

\subsection{Experimental Configuration}

The SQUID cell used for "pure NQR" detection is shown in Figure 5.5. The transmitter coil consists of a two-layer solenoid, approximately $30 \mathrm{~mm}$ long wound from $220 \mu \mathrm{m}$-diameter $\mathrm{Cu}$-coated $\mathrm{NbTi}$ wire on a $15 \mathrm{~mm}$ fiberglass tube. The pickup coil, depending on the application, consists of either a three-turn coil or a pair of two-turn coils 


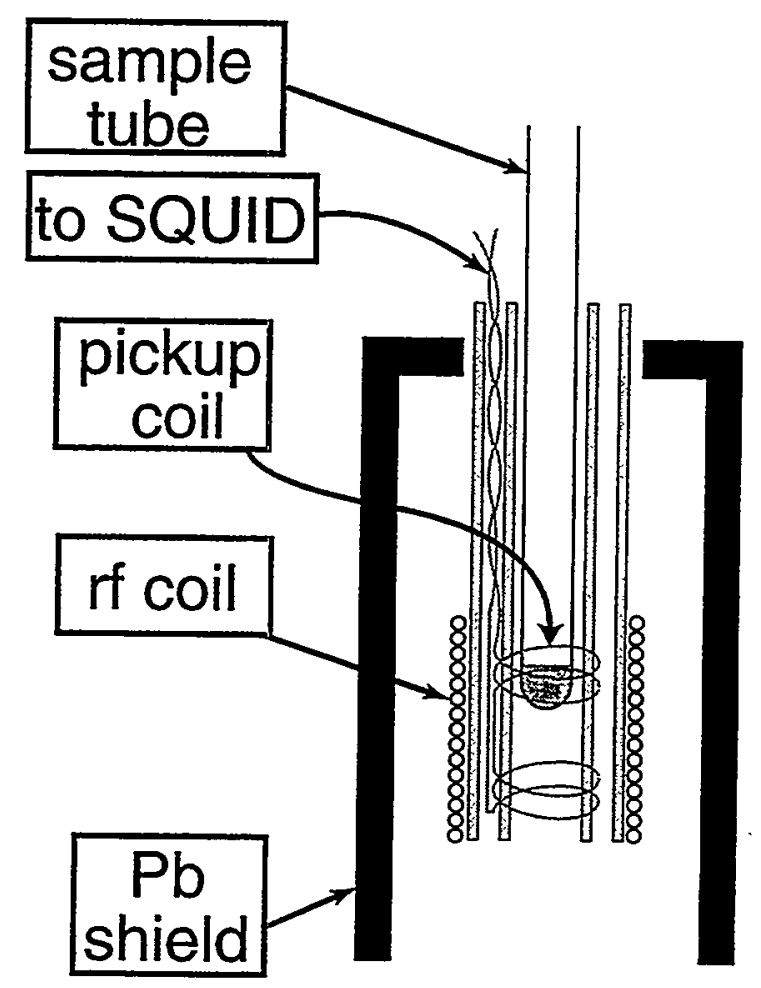

Figure 5.5: The NQR cell. The pickup coil shown consists of a pair of two-turn coils wound in a gradiometer configuration. The $r f$ coil is a multiturn solenoid.

wound in opposition as in a gradiometer configuration. I used both $1266 \mathrm{~A} \& \mathrm{~B}$ stycast and vespel as the materials to wind the pickup coil on; and the typical size of these tubes is about $8.5 \mathrm{~mm}$ diameter and $40 \mathrm{~mm}$ long. In the case of the pair of two-turn coils, their relative positions is about $15 \mathrm{~mm}$, and the whole pickup coil is adjusted relative to the transmitter to minimize the coupling. The three-turn single coil is used for higher frequencies $(>200 \mathrm{kHz})$ where the ring-down time in the transmitter circuit is short enough that strong feedthrough of the af signal is not a problem. The typical sample of $500 \mathrm{~mm}^{3}$ is packed into a $8 \mathrm{~mm}$ outer-diameter glass or pyrex tube and then inserted into the middle of the cell for detection. 


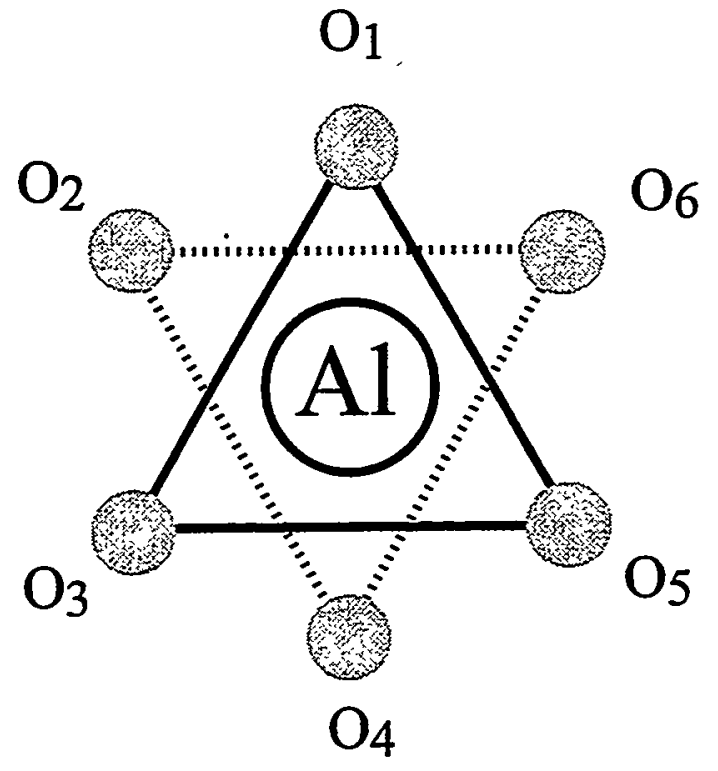

Figure 5.6: The ${ }^{27} \mathrm{Al}$ nucleus in $\mathrm{Al}_{2} \mathrm{O}_{3}$ is at the center of an octahedron formed by six oxygens atoms in a hexagonal close packed motif. The $z$ axis is perpendicular to the page, penetrating the ${ }^{27} \mathrm{Al}$ nucleus. In this configuration, the high symmetry of the EFG in the $x y$ plane leads to a vanishingly small $\eta$.

\subsection{Results}

\subsection{1 ${ }^{27} \mathrm{Al}$ in Ruby (1-D)}

The sample used in this study is $500 \mathrm{~mm}^{3}$ of $0.5 \mathrm{~mm}$-diam spheres with $7 \% \mathrm{Cr}^{3+}$. At $4.2 \mathrm{~K}$ the longitudinal relaxation time $T_{1}$ of ${ }^{27} \mathrm{Al}(\mathrm{I}=5 / 2)$ for pure sapphire $\left(\mathrm{Al}_{2} \mathrm{O}_{3}\right)$ is about 1 minute; the $\mathrm{Cr}^{3+}$ ions significantly shorten $T_{1}$, thereby substantially reducing the data acquisition time[5]. The structure and quadrupole parameters for sapphire are well know. The oxygen atoms in the unit cell adopt a hexagonal close packed motif which forces an ${ }^{27} \mathrm{Al}$ nucleus to the center of an octahedron formed by six oxygen atoms as 


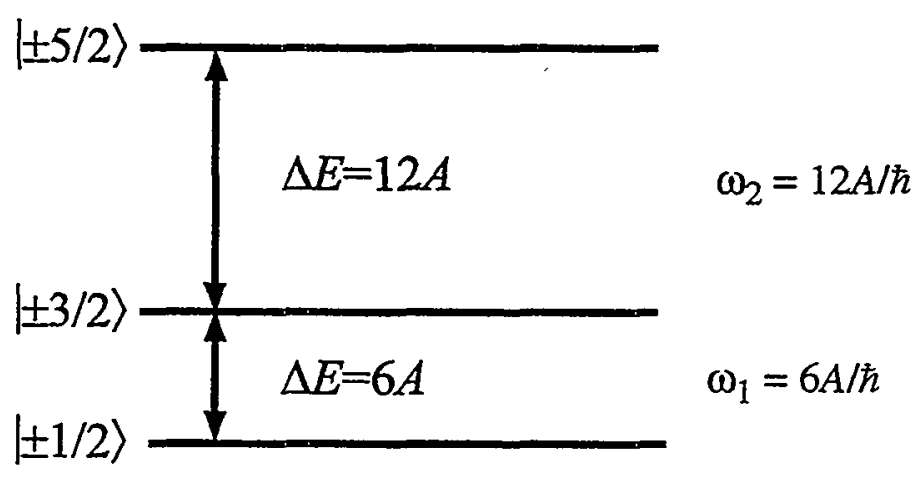

Figure 5.7: The energy levels for ${ }^{27} \mathrm{Al}(I=5 / 2)$ in sapphire. High symmetry in the $x y$ $\mathrm{EFG}$ leading to a vanishingly small $\eta$ makes three energy levels two-fold degenerate of 2 . Because of selection rules, there should be only two observable transitions, with resonance frequencies related by a factor of $2, \omega_{1}=\omega_{2} / 2=6 \mathrm{~A} / \hbar$

shown in Figure 5.6[6]. In essence, an ${ }^{27} \mathrm{Al}$ nucleus is sandwiched between two equilateral triangles of oxygen atoms rotated by $60^{\circ}$ with respect to each other. The high structural symmetry of the unit cell leads to a similar electronic symmetry, implying that $\eta$ of the ${ }^{27} \mathrm{Al}$ nuclei in sapphire should be vanishingly small. Unfortunately, one cannot use similar symmetry arguments to predict the magnitude of the quadrupolar coupling constant; to obtain a reasonably accurate estimate of this parameter one has to perform an electronic structure calculation. Since $\eta$ is nearly zero in sapphire, the $|m\rangle$ and $|-m\rangle$ states $(m=1,3$, or 5$)$ are largely degenerate in energy as calculated in Section 5.2.2. As a result there should be only two observable transitions, with resonance frequencies related by a factor of $2, \omega_{1}=\omega_{2} / 2=6 A / \hbar$, as shown in Figure 5.7.

Figures 5.8 and 5.9 show the free induction decay and the Fourier transform of the 

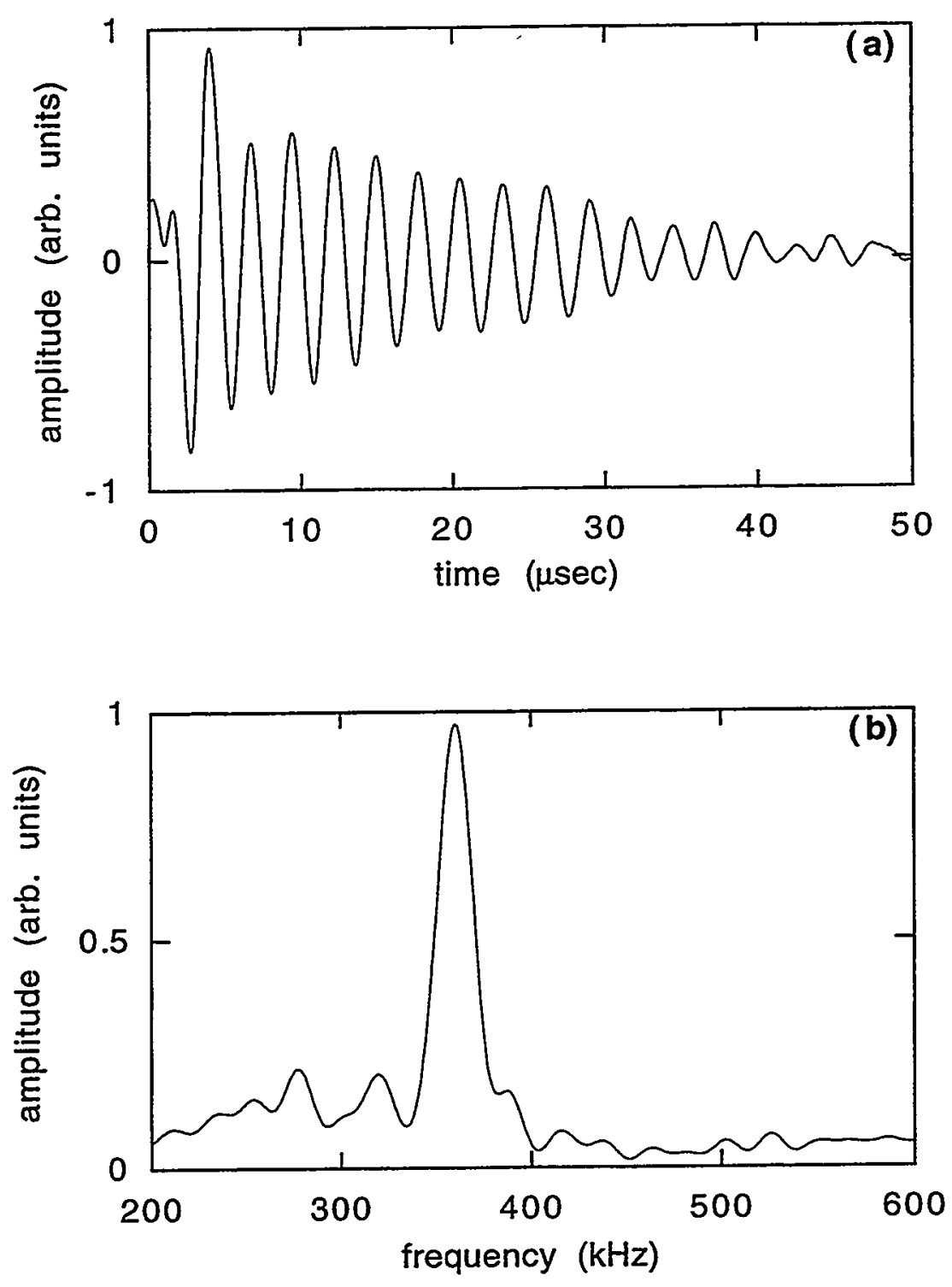

Figure 5.8: (a) Free induction deacy of the $| \pm 1 / 2\rangle \leftrightarrow| \pm 3 / 2\rangle$ in ${ }^{27} \mathrm{Al}$ in $\mathrm{Al}_{2} \mathrm{O}_{3}\left[\mathrm{Cr}^{3+}\right]$ at 4.2 (b) Fourier transform of (a) shows a peak at $359 \mathrm{kHz}$. 

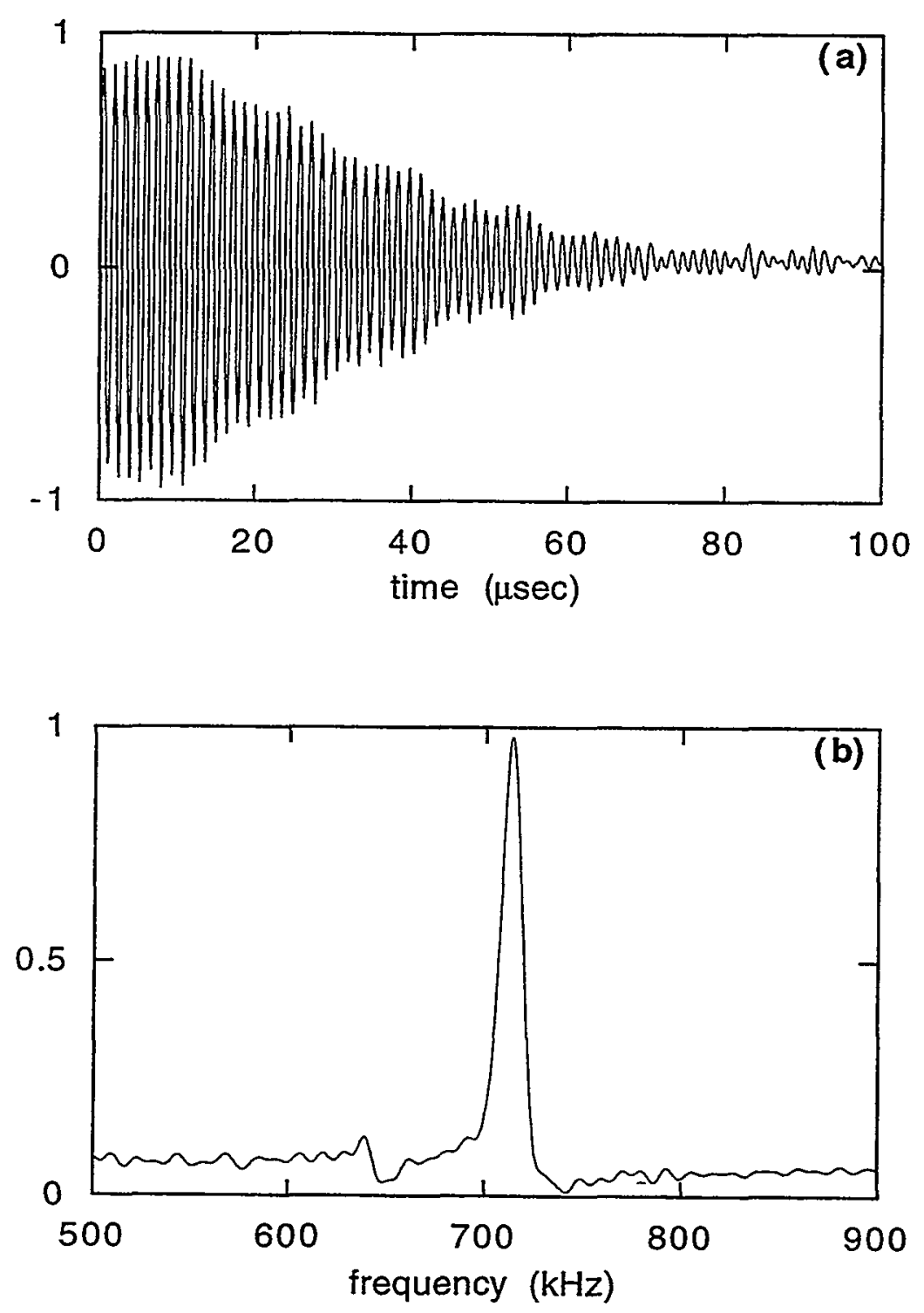

Figure 5.9: (a) Free induction deacy of the $| \pm 3 / 2\rangle \leftrightarrow| \pm 5 / 2\rangle$ in ${ }^{27} \mathrm{Al}$ in $\mathrm{Al}_{2} \mathrm{O}_{3}\left[\mathrm{Cr}^{3+}\right]$ at 4.2 (b) Fourier transform of (a) shows a peak at $714 \mathrm{kHz}$. 
two NQR lines, obtained approximately $10 \mu$ s after the magnetic pulse was turned off and averaged over 4000 scans. The magnetic field pulse was typically 10 to $20 \mathrm{kHz}$ off-resonance, ten periods in length, and with a peak-to-peak amplitude of about $2 \mathrm{mT}$. The sampling rate was $3 \mathrm{~Hz}$. The observed lines at frequencies of 359 and $714 \mathrm{kHz}$ arise from transitions between the $| \pm 3 / 2\rangle$ and $| \pm 1 / 2\rangle$, and $| \pm 5 / 2\rangle$ and $| \pm 3 / 2\rangle$, respectively, and are in good agreement with measurements reported at different temperatures and with different methods[7,8].

\subsubsection{Two-dimension NQR $-{ }^{27} \mathrm{Al}$ of Ruby}

The extension of spectroscopy to two and more dimensions stems from the recognition that the properties of a molecular system cannot be fully characterized by a conventional one-dimensional spectrum[9]. Coupled spins can complicate a typical 1-D spectra with many resonant lines, especially in a very complicated molecules such as proteins. In 2-D spectra, for example, the coupled spins are correlated, and thus 2-D NMR is helpful in identifying these coupled systems. The concept was first proposed in 1971 and since then has become one of the most powerful NMR techniques in solving problems of physics, chemistry, and biology. R. Ernst won his Nobel price for work related to multi-dimensional NMR experiments and techniques.

Hence, a further step in demonstrating the power of the pulsed SQUTD spectrometer is the extension of the experiments to more than one dimension. Two important points which made ruby a good candidate for the 2-D experiment are its rather large signal, requiring less times for averaging, and a fast $T_{1}$ relaxation time, allowing for the rapid acquisition of the 2-D data. However, $T_{2}$ is very short and thus the effect of the cross peaks in a 2-D experiment is not very strong. Nevertheless the data obtained show the capability 


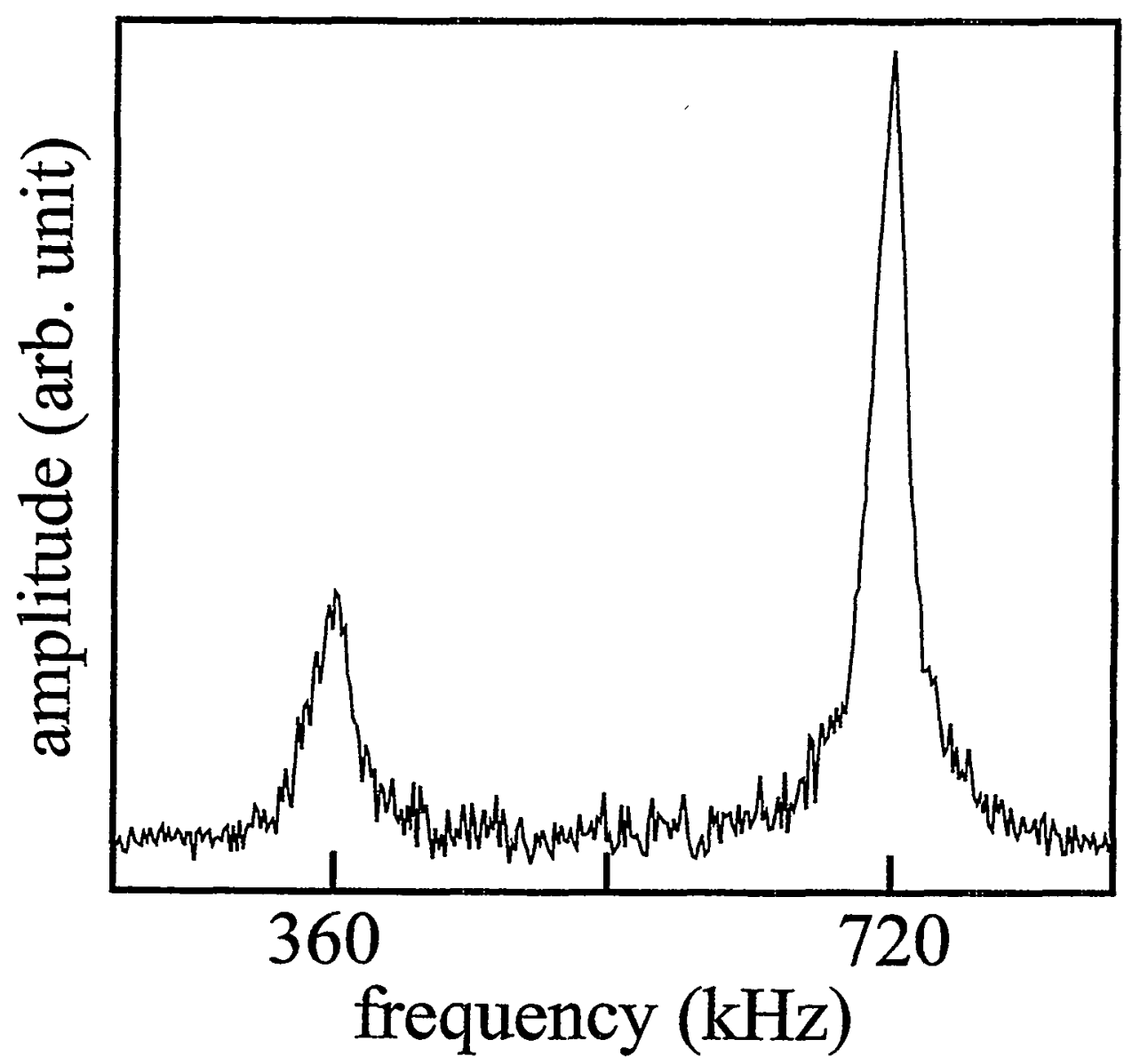

Figure 5.10: Fourier transform of the ${ }^{27} \mathrm{Al} N \mathrm{NQR}$ free induction decay with both transitions irradiated showing both peaks at 360 and $720 \mathrm{kHz}$.

of the SQUID 2-D NQR technique.

Although the pulse fields produced by the probe can be substantial, they were not sufficient to excite both $\mathrm{NQR}$ lines with a single frequency. For this reason we introduced a second function generator so that we could simultaneously excite the $359 \mathrm{kHz}$ and 714 $\mathrm{kHz}$ resonances. We gated the two synthesizers simultaneously by splitting a single TTL trigger line. Typically, we average the signal 512 times, and the data is acquired with a digital scope at the rate of $10-25 \mathrm{~Hz}$ rate. The spectrum obtained for the two-frequency pulse is shown in Figure 5.10. 


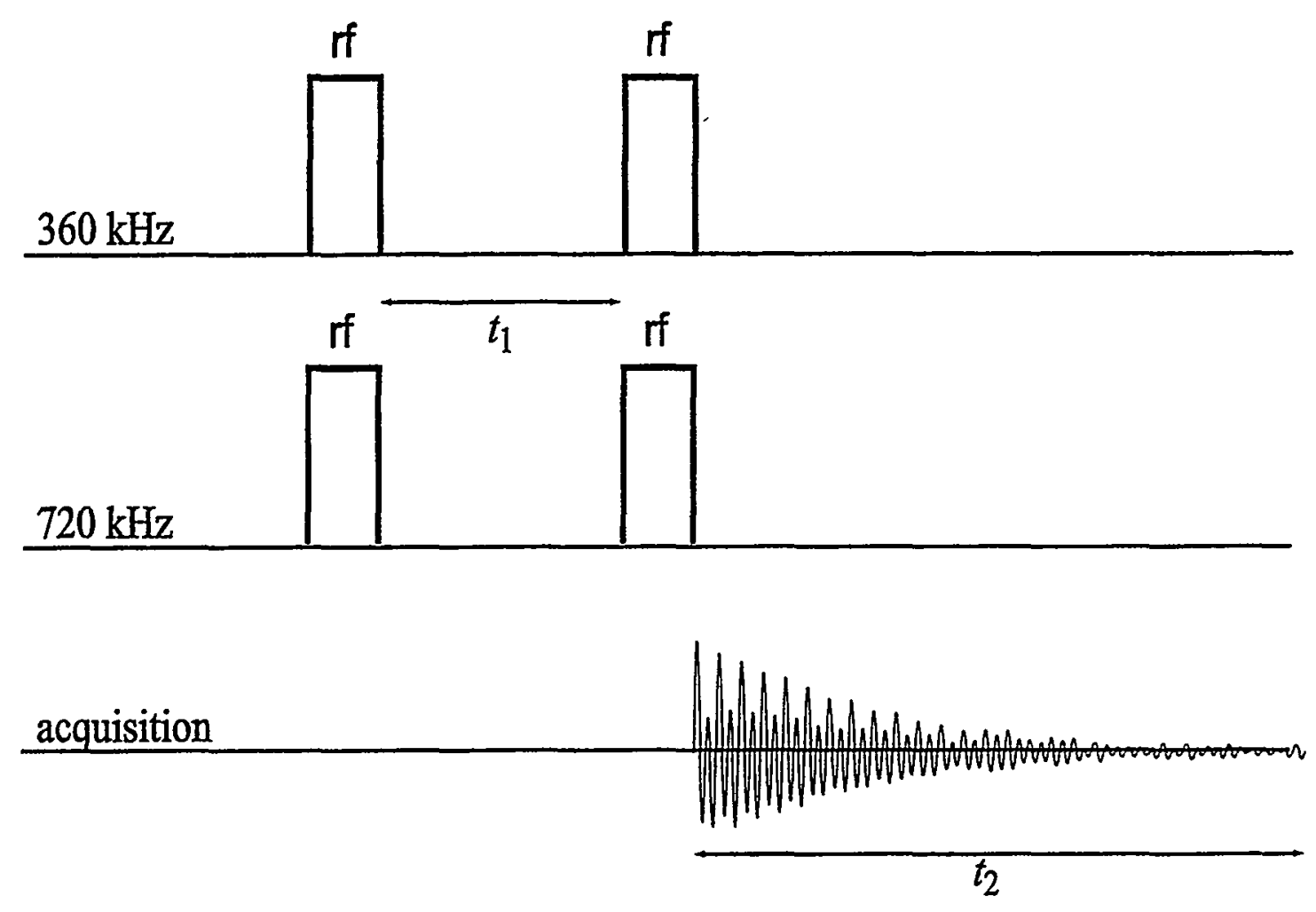

Figure 5.11: The first pair of pulses at both 359 and $714 \mathrm{kHz}$ prepares the population of energy levels in a state away from thermal equilibrium. The polarization evolves for a a time $t_{1}$ allowing a new equilibrium to form. The second pulse is a readout pulse.

It is straightforward to construct a two dimensional data set from this experiment by applying a second, two-frequency pulse a time $t_{1}$ after the first pulse, as shown in Figure 5.11. The free induction decays are collected as a function of a time $t_{2}$. The amplitudes of the FIDs at a constant $t_{2}$ oscillates as a function of $t_{1}$ at both $359 \mathrm{kHz}$ and $714 \mathrm{kHz}$. In the absence of the second, two-frequency pulse, the $359 \mathrm{kHz}$ line oscillates at its own frequency as a function of $t_{1}$. The same is true for the $714 \mathrm{kHz}$ transition. A two-dimensional Fourier transform of a data set obtained with the second pulse produces two peaks only, 


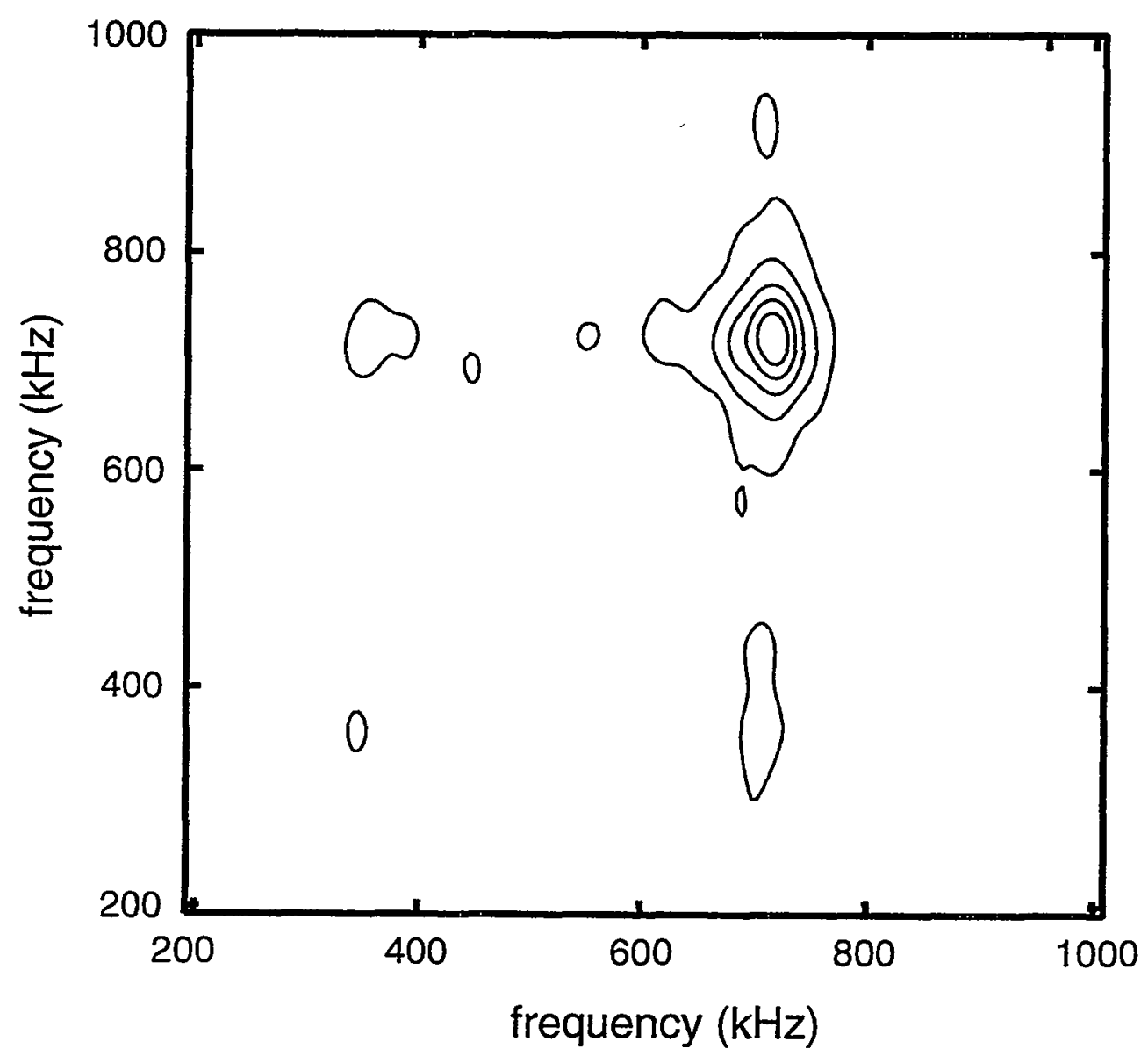

Figure 5.12: Two-dimensional NQR spectrum of ${ }^{27} \mathrm{Al}$ in ruby. The spectrum was constructed from 64 free induction decays, averaged 512 times, measured as a function of the interval between the two-frequency pulses. The time between the pulses was incremented in $0.5 \mu$ s steps.

correlating each of the frequencies $\omega_{1} / 2 \pi$ and $\omega_{2} / 2 \pi$ with itself. In the experiment presented here, the second two-frequency pulse has the net effect of mixing the populations of the two transitions at a time $t_{1}$ after the first pulse. In essence the $359 \mathrm{kHz}$ coherence as a function of $t_{1}$ is changed into a detectable $714 \mathrm{kHz}$ coherence. This leads to two extra cross peaks in the two dimensional Fourier transform correlating the $359 \mathrm{kHz}$ and 714 $\mathrm{kHz}$ lines in ${ }^{27} \mathrm{Al}$.

The results are shown in Figure 5.12. However, accurate predictions of the cross- 
peak amplitude seen in the experiment are not yet available, since detailed calculations must be performed to include the effects of the pulse intensity and orientations of the nuclei with respect to the ${ }^{27} \mathrm{Al}$ principal axis, as well as the relaxation during $t_{1}$.

This qualitative experiment is the first demonstration of two-dimensional NQR using a SQUID detector. It is hoped that the development of this technique will lead to new insights into correlating ${ }^{14} \mathrm{~N} N Q R$ spectra in biological systems. Work is currently in progress towards using the two-dimensional methods to study amino acids in biological systems. In combination with spin labeling these compounds with a nitroxol group, we hope $T_{1}$ will shorten significantly, allowing for faster signal averaging, as well as the possibility of performing dynamic nuclear polarization to enhance the signal. We hope these improvements will make the pulsed ${ }^{14} \mathrm{~N}$ NQR a viable option in the study of biological samples.

\subsection{3 ${ }^{2} \mathrm{D}$ NQR}

Deuterium NQR is probably one of the most studied quadrupolar nuclei. The method typically used is high field NMR where the ${ }^{2} \mathrm{D}$ quadrupolar interaction is just a perturbation on the Zeeman interaction of $50-100 \mathrm{MHz}$. Furthermore, for such small perturbations, a conventional Faraday detector can be tuned to allow for the acquisition of the whole ${ }^{2} \mathrm{D}$ NMR spectrum. In high magnetic field, theoretical ${ }^{2} \mathrm{D}$ NMR spectra are shown in Figure 5.13 for both single crystal and powder sample. In the case of the single crystal, to first order in the quadrupolar perturbation, there are two resonances given by

$$
v_{ \pm}=-\gamma B_{0} \pm \frac{3 A}{2 h}\left(3 \cos ^{2} \theta-1\right)
$$



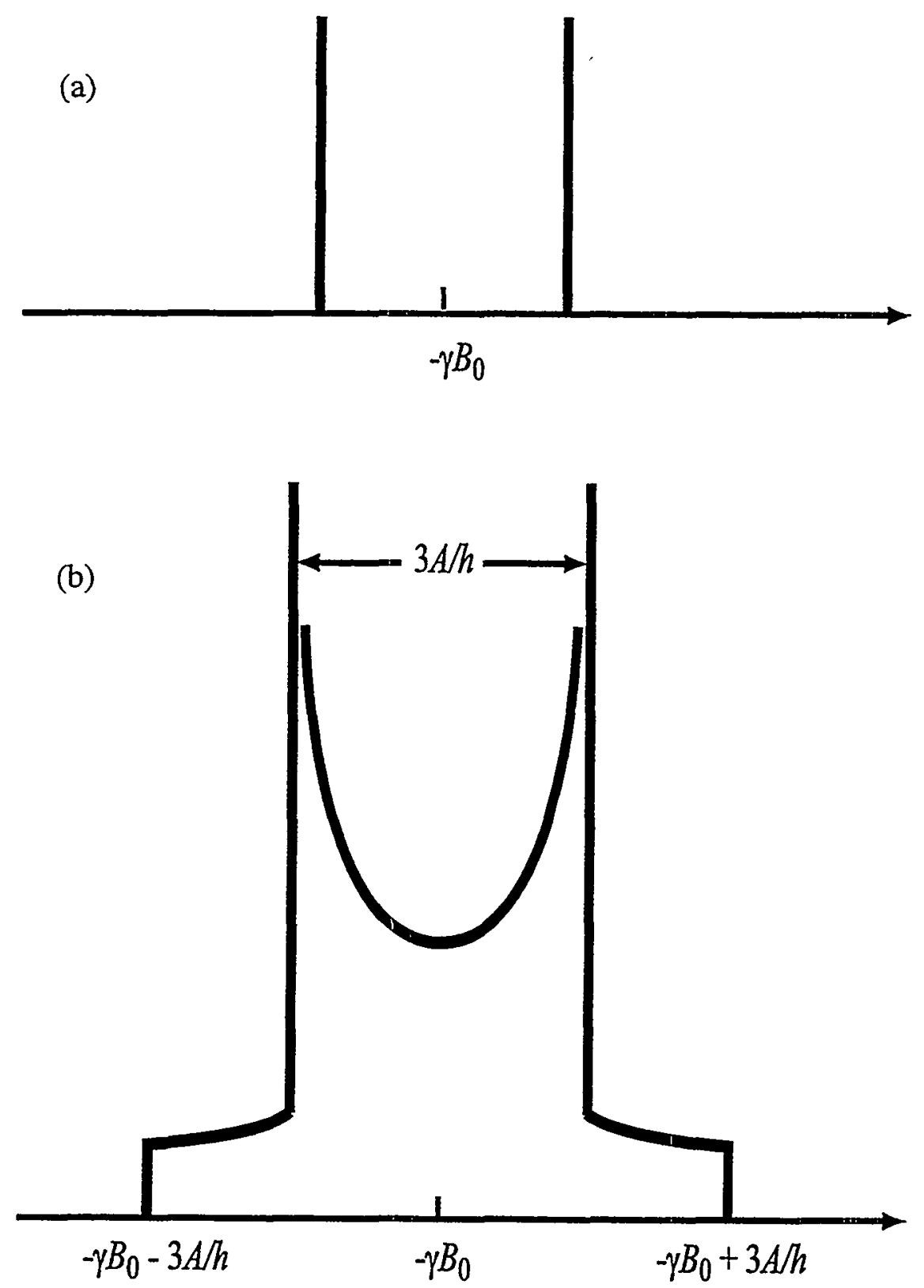

Figure 5.13: (a) For a single crystal, the high field NMR spectra of ${ }^{2} \mathrm{D}$ yields 2 lines centered at the Larmor frequency separated by $3 A\left(\cos ^{2} \theta-1\right) / h$. (b) The ${ }^{2} \mathrm{D}$ high field NMR spectra of a powder sample yield a broad spectrum. 
where $\theta$ is the angle between the direction of magnetic field and the PAS. Thus the two frequencies are centered at the Larmor frequency and are separated by $(3 A / h)\left(3 \cos ^{2} \theta-1\right)$. The average over all possible angles $\theta$ gives the "powder pattern" shown in Figure 5.13 (b).

The case is more interesting for the ${ }^{2} \mathrm{D} N \mathrm{NMR} / \mathrm{NQR}$ of the methyl $\left(\mathrm{CD}_{3}\right)$ group. The quadrupole coupling constant of the deuterium in $\mathrm{CD}_{3}$ group is of the order of $150-180$ $\mathrm{kHz}[10]$. At room temperature, the motional averaging by rapid rotation gives an asymmetry parameter $\eta \sim 0$ resulting in a pair of transitions in high field NMR and one single transition for zero field NQR (aprroximately about $40 \mathrm{kHz}$ ). The next question to ask is what will happen when the temperature is lowered so that this rapid rotation is frozen out.
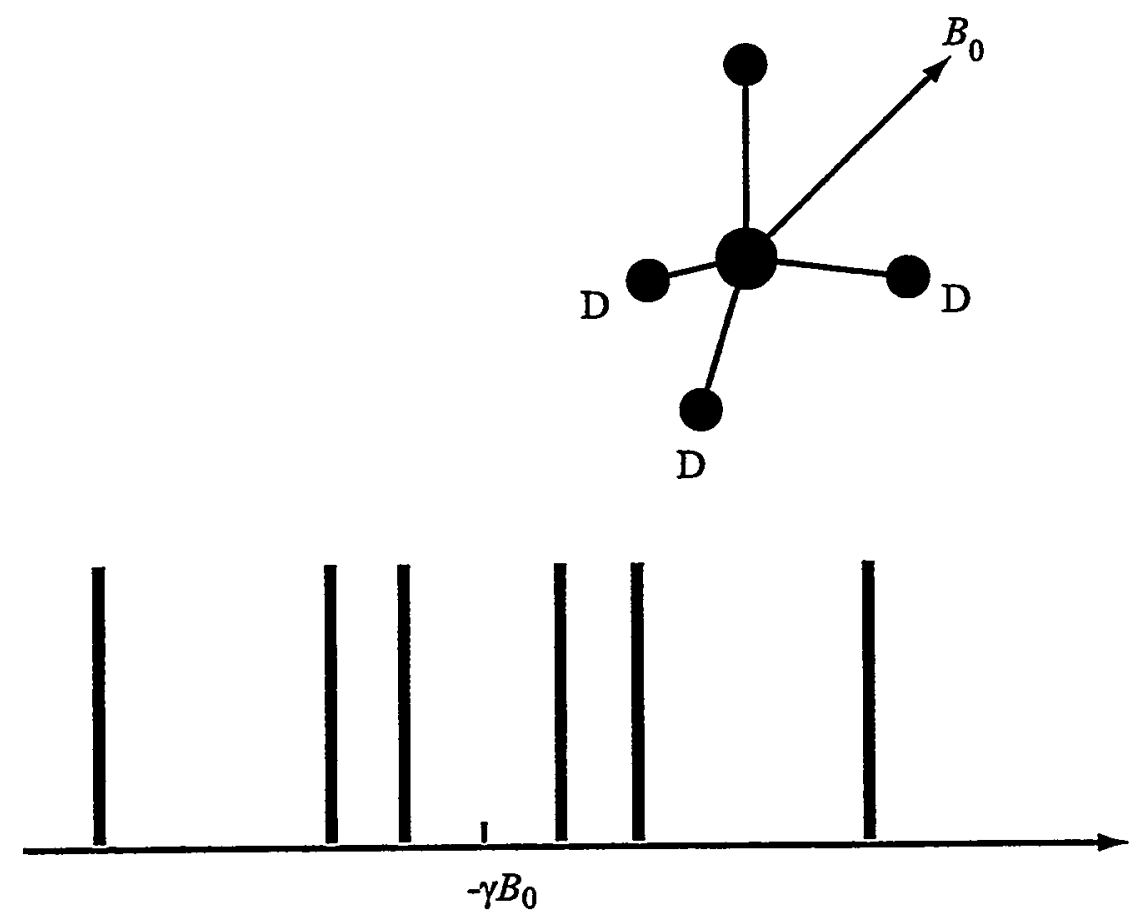

Figure 5.14: The high field NMR spectra of 2D in the methyl group in which the 2D is frozen in the lattice. Each deuterium contributes to a pair of lines, giving a total of three pair of lines of equal intensities. 
Two things can happen. The first case is that all the deuterons will become immobile in their lattice, so that each deuteron will feel its local EFG. For a single-crystal sample in high field, each deuteron will give a pair of lines resulting in three pairs of lines of equal intensity, Figure 5.14. In zero field however, each deuteron will feel its own local EFG giving it a possible three lines and thus resulting spectra with three sets of triplet. However, the deuterons in this $\mathrm{CD}_{3}$ group may tunnel between sites. Instead of the classical rotational mechanics of the high tempearture case, quantum mechanical tunneling now dominates the dynamics of the systems[11]. This tunneling is particularly interesting because of the physics involved. The information obtained can provide insight into the interatomic forces in molecular solids[12]. Also, since the tunneling is temperature dependent; hence, the nature of thermally activated processes can be studied[10-12]. Theoretically and experimentally, high field NMR studies of the deuterated methyl group have been done for various compounds. The Hamiltonian will consist of the Zeeman, tunneling, quadrupole, and dipole-dipole interactions:

$$
H=H_{Z}+H_{T}+H_{Q}+H_{D} .
$$

For most of the typical theoretical calculations, the dipolar term is neglected since it is a lot smaller than the quadrupolar term. The major work is to calculate the eigenstates and eigenvalues of the above Hamiltonian. When the Zeeman term dominates, the problem is somewhat simpler than no Zeeman term because the Hamiltonian can be factorized into smaller submatrices which can be diagonalized individually. In zero-field however, the whole $27 \times 27$ matrix must be diagonalized. I will present the result on the $\mathrm{CD}_{3}$ tunneling data of the perdeuterated picoline and toluene in zero field and then discuss a little bit about them since the detail of the calculation is still ongoing. 


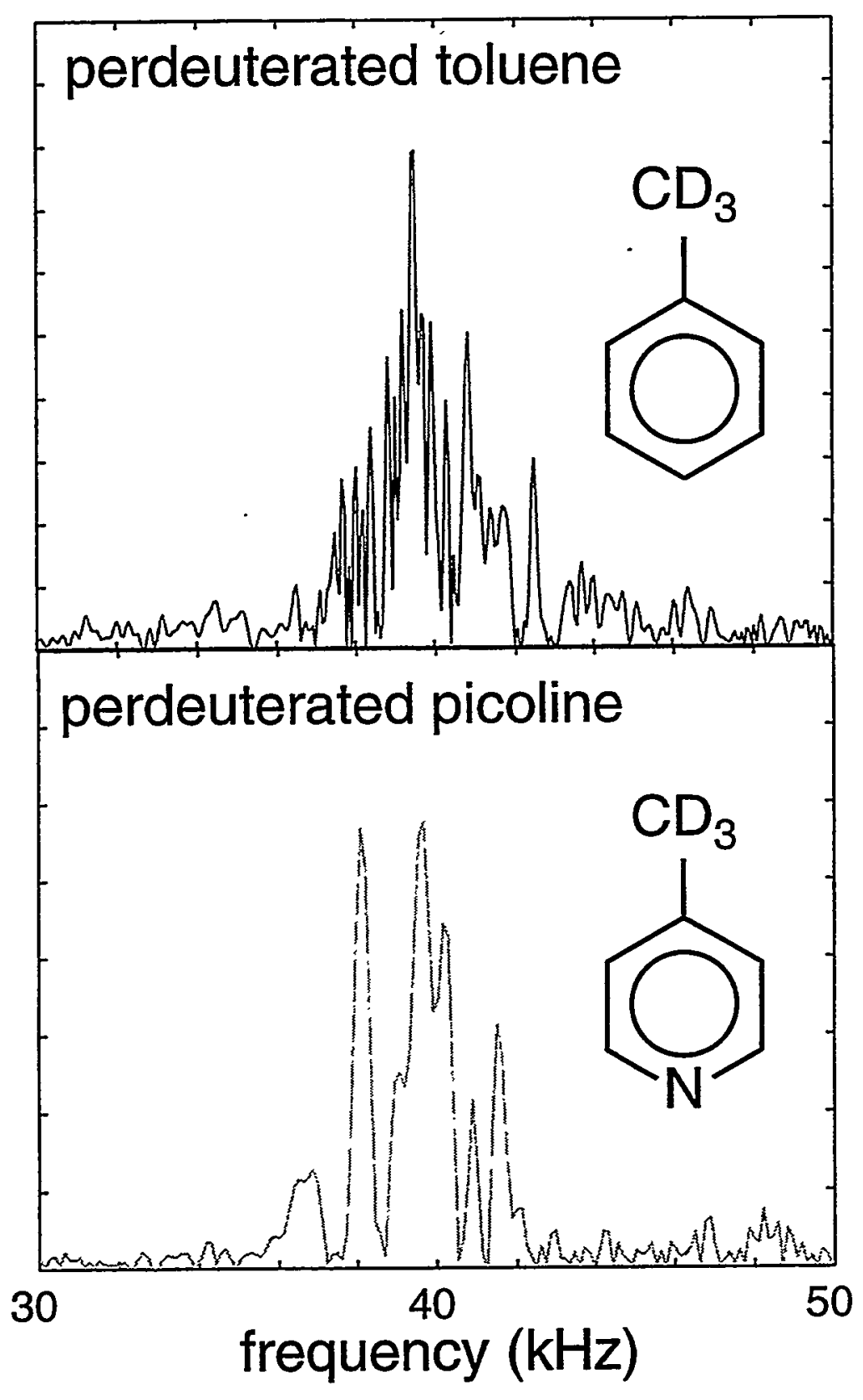

Figure 5.15: ${ }^{2} \mathrm{D}$ NQR spectrum at zero field of perdeuterated toluene and perdeuterated picoline. 
At room temperature, picoline and toluene are liquid. Upon freezing, random crystallites are formed thus giving a powder like sample. This is also a reason why we want to look at these compounds specificly in zero field. Even though, the spectra of powder sample can be obtained with high field technique, the broadening can conceal important information within the spectrum. The spectrum obtained for picoline and toluene are shown in Figure 5.15 taken at $1.2 \mathrm{~K}$ and averaged for approximately about 20000 times. We used the echo sequence with the amplitude of few Gauss and with the delay of $0.5 \mathrm{~ms}$. The thing one notices is that the spectrum is centered about $40 \mathrm{kHz}$ which is about the frequency if the $\mathrm{CD}_{3}$ group is allowed to rotate freely. This is explained by the static effect of the distribution of of the totally symmetric wavefunction over all three deuterium sites with equal probabilities[11]. The second thing to notice is that the toluene spectra shows more resonances than that of picoline. A possible reason is that toluene can has many different $\mathrm{CD}_{3}$ sites and even within one phase[13], namely in the $\alpha$-crystallized phase of perdeuterated toluene, two (and possibly more) distinct methyl groups exist giving a lot more lines. Nevertheless, from the previous argument the ${ }^{2} \mathrm{D}$ toluene spectrum clearly show tunneling within the $\mathrm{CD}_{3}$ group. In order to help grouping the resonances to a particular methyl group, one might attempt to perform 2-D NQR, Section 5.4.2, to correlate the resonances. The picoline spectra is still under investigation. Attempts are under way to theoretically predict the tunneling rate of the deuterons. 


\section{References}

[1] H. Chihara and N. Nakamura, Nuclear Quadrupole Resonance Spectroscopy Data Vol 20, (Spinger-Verlag, Berlin, 1988).

[2] J.A.S. Smith, Advances in Nuclear Quadrupole Resonance (Heyden, London, 1974).

[3] N. Q. Fan, Ph. D. Thesis, University of California, Berkeley, California.

[4] T.P. Das and E.L. Hahn, Solid State Supplement 1 : Nuclear Quadrupole Resonance Spectroscopy, (Acadamic Press, New York, 1958.)

[5] C.M. Verber, H.P. Mahon, W.H. Tantilla, Phys. Rev. 125 (1962) 1149.

[6] N. Ishizawa, T. Miyata, I. Minato. F. Marumo, S. Iwai, Acta Cryst. B 36 (1980) 228.

[7] R.V. Pound, Phys. Rev. 79 (1950) 685.

[8] C. Connor, J. Chang. A. Pines. J. Chem. Phys. 93 (1990) 7639.

[9] R.R. Ernst, G. Bodenhausen, and A. Wokaun, Principles of Nuclear Magnetic Resonance in One and Two Dimensions, (Clarendon Press, Oxford, 1992.)

[10] Z. T. Lalowicz, U. Werner, and W. Muller-Warmuth, Z. Naturforsch. 43a (1988) 219.

[11] Z. Olejniczak, A. Detken, B. Manz, U. Haeberlen, J. Mag. Res. A 118 (1996) 55.

[12] A. Detken, P. Focke, H. Zimmermann, U. Haberlen, Z. Olejniczak, and Z.T. Lalowics, Z. Naturforsch. 50a (1994) 95.

[13] D. van der Putten, G. Diezemann, F. Fujara, K. Hartmann, H. Sillescu, J. Chem. Phys. 96 (1992) 1748. 


\section{Chapter 6}

\section{Dynamic Nuclear Polarization at Low Field}

\subsection{Introduction}

As mentioned earlier, the signal-to-noise ratio (SNR) in a solid-state NMR experiment is very low. The use of a SQUID (Chapter 2) and optical pumping process (Chapter 3 and 4) are two techniques discussed so far to tackle this SNR problem. The signal enhancement in an optical pumping experiment is potentially enormous; however, there are many drawbacks to using such technique. Generally, very few systems can have their spin polarization increased in this way. For example, the high ionization potential of rubidium atoms has limited the optical-pumping/spin exchange method to noble gases such as ${ }^{129} \mathrm{Xe}$ and ${ }^{3} \mathrm{He}$. Usage of highly polarized ${ }^{129} \mathrm{Xe}$ to significantly enhance other nuclei has been successfully in a few-limited experiments[1,2,3,4,5]. For an efficient transfer of the ${ }^{129} \mathrm{Xe}$ spin polarization to other nuclei the spin relaxation time $T_{1}$ of these nuclei must be long[2,3]. Additionally, in order for such polariztion to be transferred to 
solids, the sample must have a large surface area that can absorb ${ }^{129} \mathrm{Xe}[4,5]$. Often, these requirements limit the generality of such experiments.

In thinking of a solution to such problems, the aim is to perform new kinds of SQUID-detected experiments with sufficient sensitivity and a faster acquisition time. One technique that has been successfully applied in an NMR experiment to increase the signal is a scheme employing the Overhauser Effect. In systems containing free electrons such as metals, or samples with paramagnetic centers, the nuclear spin can be increased by a factor of 1000 over thermal equilibrium if the electron channel is saturated[6]. This idea was suggested by Overhauser while still a graduate student at Berkeley. This concept has now been expanded to coupled-spin systems to study low spin density nuclei by observing the higher spin density nuclei coupled to them. The advantages of performing dynamic nuclear polarization (DNP) experiments in conjunction with a SQUID spectrometer setup are two-fold. The first advantage is the large enhancement in the signal offered by DNP, and the second is the short nuclear relaxation time $T_{1}$ of these electron-containing systems. The shortened $T_{1}$ caused by relaxation processes via the electrons reduces the time for data acquisition and also allows the possibility of performing various multi-dimensional techniques. One can imagine in systems such as proteins, where many ${ }^{14} \mathrm{~N}$ sites give rise to the resonances in a spectrum, the task of assigning the resonances of a particular site can be enormous. Performing a 2-D NQR on these systems may allow a better way to group these related resonances.

In this chapter, after discussing the theory of the Overhauser Effect, I will present the results of a DNP experiment done on a water containing manganese chloride in a $25 \mathrm{G}$ field with a typical Faraday detector. The goal is to combine DNP with a SQUID setup 
which I will discuss later in the chapter.

\subsection{Theory}

The concept enhancement of nuclear polarization via Overhauser Effect can be understood by discussing the simplified system consisting of a nucleus with spin $I=1 / 2$ coupled to an electron of $\operatorname{spin} S=1 / 2$, acted on by an external field $B_{0}$. The total Hamiltonian for this system is[6]

$$
H=\gamma_{e} \hbar B_{0} S_{z}-\gamma_{n} \hbar B_{0} I_{z}+A I . S,
$$

where the first term is the Zeeman interaction of the electron, the second term is the Zeeman interaction of the nucleus, and the last term is the electron-nuclear interaction with a strength denoted as $A$. In the strong field approximation, $A \ll \gamma_{e} \hbar B_{0}$. This assumption truncates the Hamiltonian with respect to $S_{z}$ :

$$
H=\gamma_{e} \hbar B_{0} S_{z}-\gamma_{n} \hbar B_{0} I_{z}+A S_{z} I_{z}
$$

Here, $m_{s}$ and $m_{i}$, the eigenvalues of $S_{z}$ and $I_{z}$, respectively, are good quantum numbers for

(a)

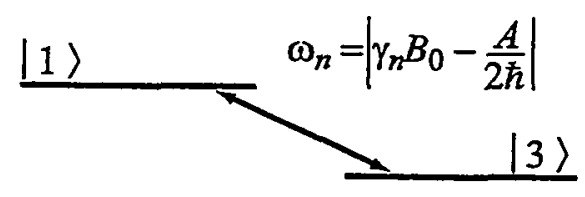

12)

$$
\omega_{n}=\left|\gamma_{n} B_{0}+\frac{A}{2 \hbar}\right|
$$

|4〉 (b)

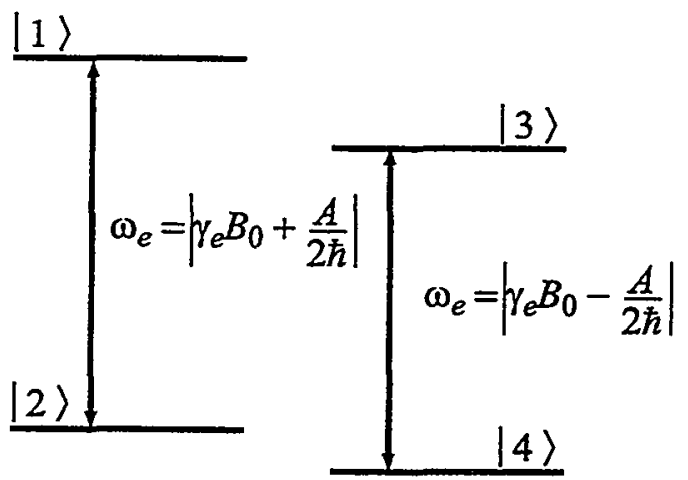

Figure 6.1: The energy level diagram of the electron-nucleus system. There are four possible levels and transitions for a nuclear spin of $1 / 2$. Two of the transitions corresponding to nuclear spin resonances (a) and the other corresponding to electron spin resonances (b). 
the Hamiltonian. For brievity, the states are labeled as followed:

$$
\begin{aligned}
& |1\rangle=\left|m_{s}=\frac{1}{2}, m_{i}=\frac{1}{2}\right\rangle \\
& |2\rangle=\left|m_{s}=-\frac{1}{2}, m_{i}=\frac{1}{2}\right\rangle \\
& |3\rangle=\left|m_{s}=\frac{1}{2}, m_{i}=-\frac{1}{2}\right\rangle \\
& |4\rangle=\left|m_{s}=-\frac{1}{2}, m_{i}=-\frac{1}{2}\right\rangle .
\end{aligned}
$$

The selection rules for the transitions are $\Delta m_{s}= \pm 1$ and $\Delta m_{i}=0$ or $\Delta m_{s}=0$ and $\Delta m_{i}= \pm 1$. As shown in Figure 6.1, there are four possible energy transitions with resonance frequencies $\omega_{e}$ and $\omega_{n}$ given by

$$
\begin{aligned}
& \omega_{e}=\left|\gamma_{e} B_{0} \pm \frac{A}{2 h}\right|, \\
& \omega_{n}=\left|\gamma_{n} B_{0} \pm \frac{A}{2 h}\right| .
\end{aligned}
$$

Here, $\omega_{e}$ and $\omega_{n}$ correspond to the electron spin resonance transitions and the nuclear spin

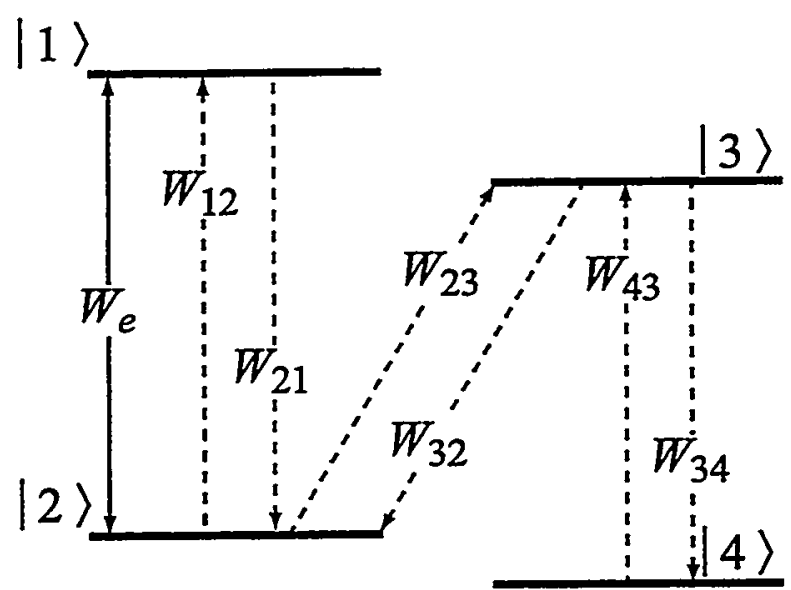

Figure 6.2: The energy level diagram of the electron-nuclear system showing different relaxation rates between energy levels. $W_{i j}$ is the transistion rate between the $i$ and $j$ energy levels and $W_{e}$ is the excitation rate applied by an external if magnetic field. 
resonance transitions, respectively. Assuming the main relaxation rates are shown in Figure 6.2 , the equations governing the populations, $\rho_{i}(i=1 . .4)$, of each energy level are

$$
\begin{aligned}
& \frac{d \rho_{1}}{d t}=\rho_{2} W_{21}-\rho_{1} W_{12}+\left(\rho_{2}-\rho_{1}\right) W_{e}, \\
& \frac{d \rho_{2}}{d t}=\rho_{1} W_{12}-\rho_{2} W_{21}+\left(\rho_{1}-\rho_{2}\right) W_{e}+\rho_{3} W_{32}-\rho_{2} W_{23}, \\
& \frac{d \rho_{3}}{d t}=\rho_{2} W_{23}-\rho_{3} W_{32}+\rho_{4} W_{43}-\rho_{3} W_{34}, \\
& \frac{d \rho_{4}}{d t}=\rho_{3} W_{34}-\rho_{4} W_{43},
\end{aligned}
$$

with

$$
\sum_{k} \rho_{k}=1
$$

Here, $W_{i j}$ are the thermally induced transition rates between the $i$ and $j$ levels, and $W_{e}$ is the pump rate applied to the electron channel. The essential idea is to calculate the nuclear magnetization $\left\langle I_{z}\right\rangle$ in the steady state as $d \rho_{i} / d t \rightarrow 0$, where

$$
\left\langle I_{z}\right\rangle=\sum_{k} \rho_{k}\left\langle k\left|I_{z}\right| k\right\rangle=\frac{1}{2}\left(\rho_{1}+\rho_{2}-\rho_{3}-\rho_{3}\right)
$$

The thermal transition rates $W_{i j}$ can be written as

$$
W_{i j}=B_{i j} W_{j i} \text {, }
$$

where $B_{i j}$ is the Boltzmann ratio $\left(B_{i j}=\exp \left(E_{i}-E_{j}\right) / k T\right)$. In the high temperature limit (which normally holds in the case of NMR),

$$
B_{i j} \cong 1+\frac{E_{i}-E_{j}}{k T}
$$


The nuclear magnetization $\left\langle I_{z}\right\rangle$ is then calculated to be

$$
\left\langle I_{z}\right\rangle \approx \frac{\hbar B_{0}}{8 k T} \cdot \frac{\zeta \gamma_{e}+2 \gamma_{n}}{\zeta+1}
$$

where

$$
\zeta=\frac{W_{e}}{W_{21}}
$$

For large $\zeta$, which is the case of saturation of the electron spin resonance,

$$
\left\langle I_{z}\right\rangle_{s a t}=\frac{\hbar B_{0} \gamma_{e}}{8 k T}
$$

and for $\zeta \ll 1$, the case of thermal equilibrium,

$$
\left\langle I_{z}\right\rangle_{\text {therm }}=\frac{\hbar B_{0} \gamma_{n}}{4 k T}
$$

Thus, one can see that upon saturating the electron spin resonance, the nuclear magnetiza-

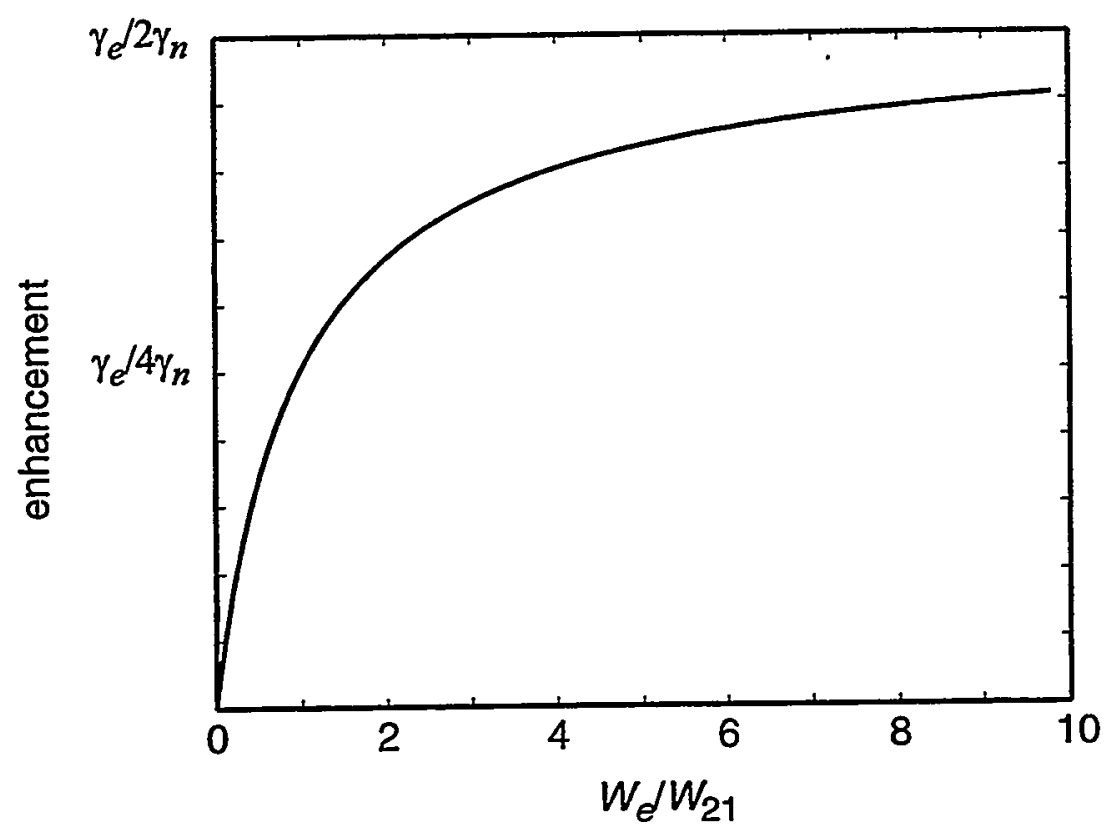

Figure 6.3: The enhancement of the nuclear magnetization as a function of the ratio of the pumping rate $W_{e}$ over the thermal transition rate $W_{21}\left(W_{e} / W_{21}\right)$. 
tion is increased by a ratio of $\gamma_{e} / 2 \gamma_{n}$. As can be seen in Figure 6.3, the enhancement reaches $\gamma_{e} / 4 \gamma_{n}$ even when the pumping rate $W_{e}$ is about the same as the relaxation time $W_{21}$.

\subsection{Experimental Setup}

\subsubsection{Probe}

Even though the goal of this project is to perform DNP using a SQUID spectrometer, the first step is to perform DNP at low field using a Faraday coil detector. The probe head shown in Figure 6.4 is based largely on an earlier double resonance design[7]. The

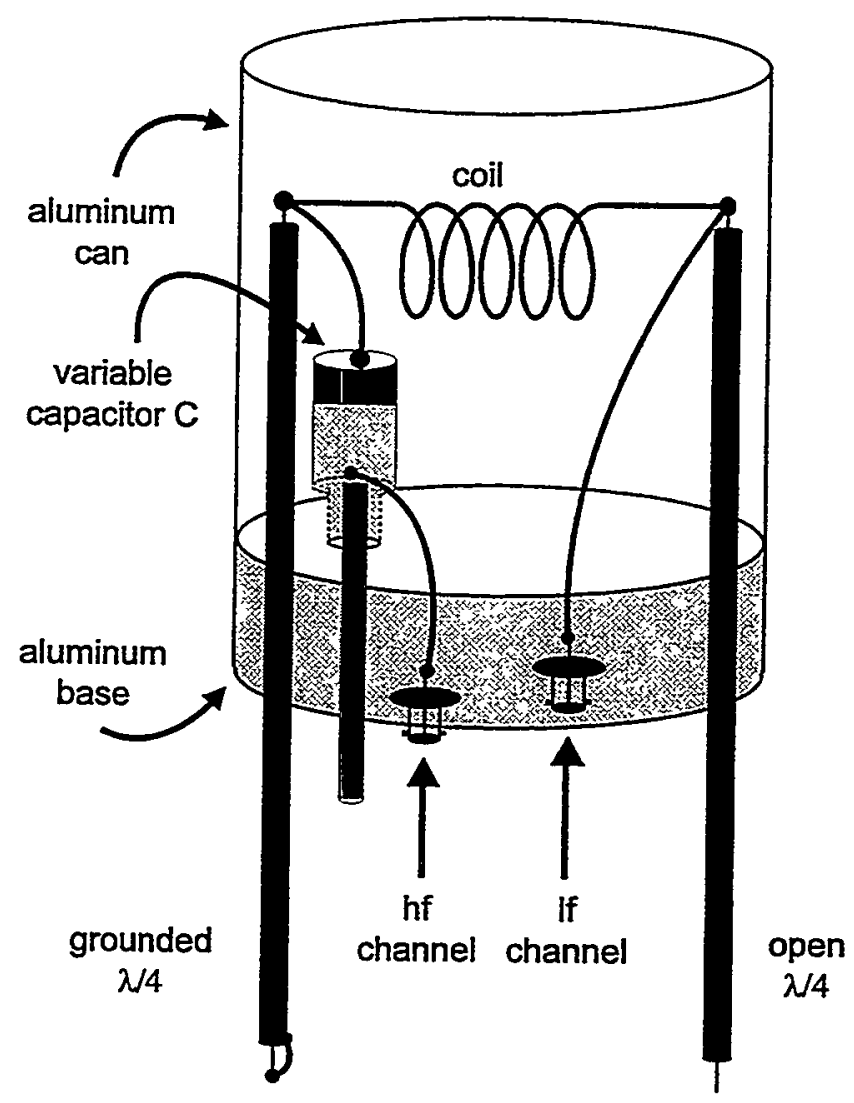

Figure 6.4: Configuration of the two-channel probe used for the low field Overhauser Effect experiment showing positions of the various components. 
circuit consists of an eight-turn solenoid, $22 \mathrm{~mm}$ long and $14 \mathrm{~mm}$ in diameter, in series with a Polyfon variable capacitor (5-25pF) for matching, and two quarter-wavelength ( $\lambda$ 4) coaxial cables. The coil is wound from enameled copper wire (1.2mm diameter) and has a volume of about $3.4 \times 103 \mathrm{~mm}^{3}$ and an inductance of $0.69 \mu \mathrm{H}$. The two $\lambda / 4$ lines consist of $50 \Omega$ semi-rigid coaxial cable with an outer diameter of $6 \mathrm{~mm}$. The whole assembly is attached to a $25 \mathrm{~mm}$ thick, 72 -mm-diam aluminum base and enclosed in a cylindrical aluminum can. This geometry allows for diagnostic tests in a $7.04 \mathrm{~T}$ superconducting solenoid magnet, which has an $89 \mathrm{~mm}$ room temperature bore. The number of turns of the solenoid is chosen so that it is self-resonant near $75 \mathrm{MHz}$, the electron Larmor frequency at a magnetic field of $2.7 \mathrm{mT}$. The series capacitor $\mathrm{C}$ is set to match the circuit to $50 \Omega$ at $75 \mathrm{MHz}$, and the length of the coaxial lines is adjusted to be $\lambda / 4$ at that frequency. The open line at the radio frequency ( $r f$ provides a path to ground for the coil while at the audio frequency ( $a f$ ) it has no effect since its impedance is high. The closed line behaves in the opposite way. Its impedance at $r f$ is large, and has a negligible effect on the tuned circuit, while at lower frequencies $(<1 \mathrm{MHz})$ the shorted cable grounds the coil.

\subsubsection{Spectrometer and Duplexer}

A block diagram of the spectrometer and duplexer is shown in Figure 6.5. The pulse gating and data acquisition are accomplished by a Techmag Aries pulse programmer. The time within a given sequence is determined by a Stanford Research Systems DG 535 pulse generator. The logic output of this unit controls both the $f$ and af channels, and

a JFET switch. The $r f$ pulses at about $75 \mathrm{MHz}$ are produced by switching the output of a PTS-500 oscillator with a GaAs-based if switch ( $~ 9$ ns ON/OFF). After passing through a Kay 432D variable attenuator, the pulses are amplified by an ENI LPI-10 amplifier to 


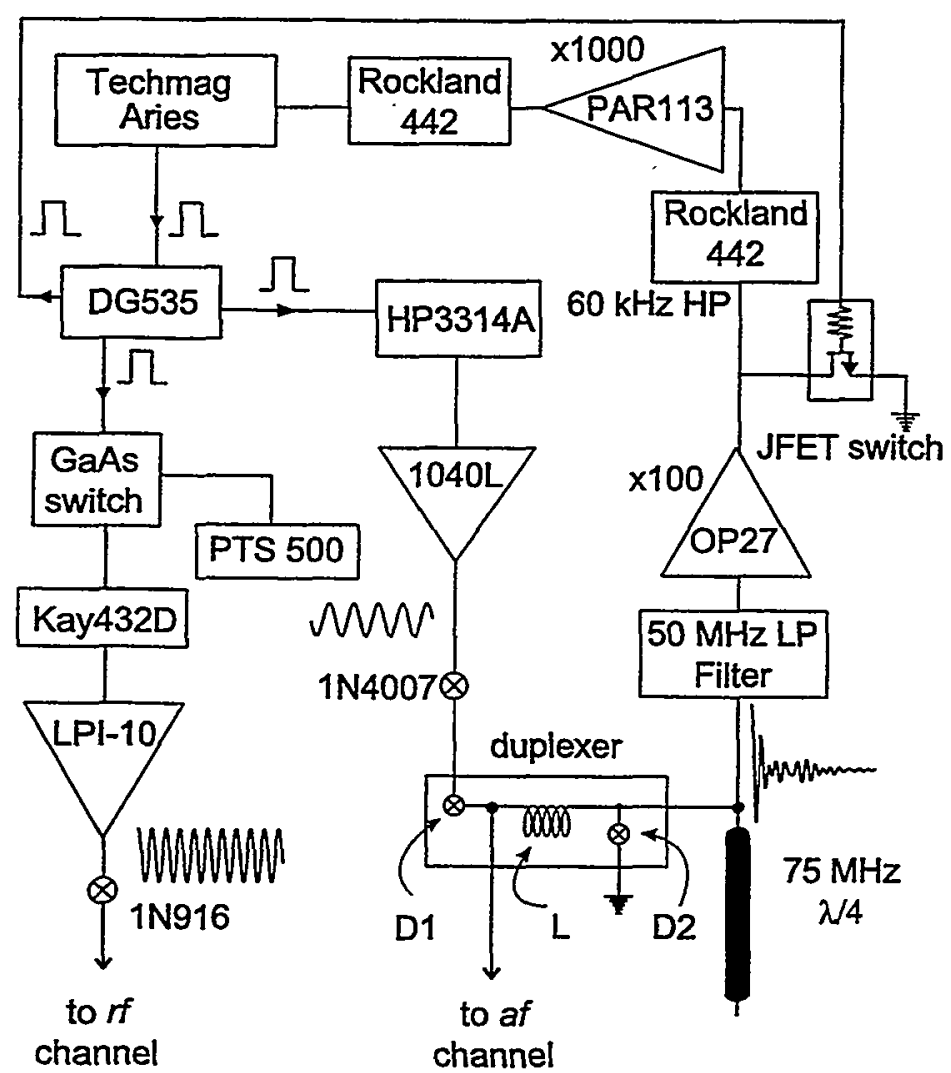

Figure 6.5: Configuration of the spectrometer.

power levels up to $1.5 \mathrm{~kW}$ (into $50 \Omega$ ). The noise of the amplifier in the absence of pulses is minimized by connecting the output to the $r f$ probe channel via a set of series crossed diodes (1N916). The high power ( 500 W, $50 \Omega)$ af pulses are produced by amplifying the output of an HP 3314A frequency synthesizer with an ENI 1040L amplifier. A set of series crossed diodes (1N4007) between the amplifier and the probe minimize amplifier noise during data acquisition.

The action of the spectrometer is as follows. During the of pulse, the two $\lambda / 4$ lines ensure that little energy is coupled into the af channel; any such leakage is attenuated by a third, open $\lambda / 4$ line and a passive, $50 \mathrm{MHz}$ low pass filter, with a $35 \mathrm{~dB}$ attenuation at 75 
$\mathrm{MHz}$, that follow the duplexer. After the $r$ pulse is turned off, the af pulse is turned on and is isolated from the detection circuit in the following way. During the pulse, diode D1 turns on, providing a low-impedance path (about $0.3 \Omega$ ) to the probe channel. The diode D2 also turns on and, together with the inductance $\mathrm{L}$ of the duplexer $(1.3 \mu \mathrm{H})$, limits the output of the duplexer to about $0.7 \mathrm{~V}_{\mathrm{rms}}$. Further attenuation of the af pulse is provided by the MTO55EL JFET in Figure 6.5, which is switched to ground during the af pulse. After the af pulse is removed, diodes D1 and D2 turn off, and the FID signal is coupled via the inductor $\mathrm{L}$ and low pass filter to the OP27 amplifier. The signal is high pass filtered to the OP27 amplifier. The signal is high pass filtered at $60 \mathrm{kHz}$ by a Rockland 442 filter, amplified by a PAR 113 amplifier and passed through a (70-250 $\mathrm{kHz})$ bandpass filter before being digitized by the Techmag.

A static magnetic field up to $20 \mathrm{mT}$ could be applied by means of a solenoid $0.3 \mathrm{~m}$ in diameter and $0.6 \mathrm{~m}$ long, driven by an HP $6253 \mathrm{~A}$ power supply. The field at the center of this magnet was calibrated using electron paramagnetic resonance in a single crystal of the radical cation salt fluoranthene antimony hexaflouride[8].

\subsection{Result and Discussion}

\subsubsection{Performance}

Two important criteria for the probe are the isolation of the two channels from each other and the amplitude $B_{1}$ of the $r f$ magnetic field for a given input $r f$ power. The channel isolation is determined by connecting each input of the probe in turn to a source at the appropriate frequency and measuring the signal leakage at the other input. The $r f$ isolation, $51 \mathrm{~dB}$, is determined largely by the accuracy $(\sim 1 \%)$ with which we can adjust the length of 
the two coaxial lines. The af isolation $(80 \mathrm{~dB})$ is determined by the high impedance of the matching capacitor and the low impedance of the grounded $\lambda / 4$ line. Although the length of this line is not important for the af isolation, significant departures of its length from $\lambda / 4$ at the high frequency can result in substantial heating since the $r f$ is not totally reflected. Thus, it is highly advantageous to adjust the length of the line as accurately as possible to $\lambda / 4$ since the $f$ energy is then converted mostly into magnetic field rather than heat.

The amplitude $B_{1}$ of the $75 \mathrm{MHz}$ field is calibrated using the ${ }^{79} \mathrm{Br}$ line in powdered potassium bromide at 7.04T. At an $r$ power setting that delivered $1.1 \mathrm{~kW}$ into a $50 \Omega$ load, the $90^{\circ}$ pulse length $t_{90}$ was $5 \mu \mathrm{s}$, yielding $B_{1}=1 / 4 \gamma_{\mathrm{Br}} \mathrm{t}_{90}=0.65 \mathrm{mT}$; $\gamma_{\mathrm{Br}}$ is the gyromagnetic ratio for ${ }^{79} \mathrm{Br}$. A rough estimate of $B_{1}$ in the af channel was made by measuring the voltage $V_{0}$ from a solenoid with $\mathrm{n}=6$ turns and a diameter $\mathrm{d}=5 \mathrm{~mm}$ placed inside the coil shown in Figure 6.4. At low frequency, we estimate $B_{1} \approx 4 V_{0} / \mathrm{n} \pi \mathrm{d}^{2} \omega$, where $\omega$ is the applied angular frequency. For $\omega / 2 \pi=120 \mathrm{kHz}, V_{0}=20 \mathrm{mV}$ yields $B_{1} \approx 0.2 \mathrm{mT}$. This value is refined by measuring the $90^{\circ}$ pulse length of the protons following a gated DNP experiment on the manganese (II) chloride and water solution $\left(\mathrm{MnCl}_{2} / \mathrm{H}_{2} \mathrm{O}\right)$ discussed below. This measurement yielded $\mathrm{t}_{90}=50 \mu \mathrm{s}$ and hence $B_{1}=0.12 \mathrm{mT}$.

\subsubsection{Pulsed DNP}

The sample chosen for the DNP experiment is $\mathrm{MnCl}_{2} / \mathrm{H}_{2} \mathrm{O}$ solution, primarily because the $\Delta \mathrm{F}=0, \Delta \mathrm{m}_{\mathrm{F}}=-1$ hyperfine transition energies in $\left[\mathrm{Mn}(\mathrm{H} 2 \mathrm{O})_{6}\right]^{2+}$ at $B_{0}=2.7$ $\mathrm{mT}$ are nearly those of the free electron Larmor frequency[9]. Furthermore, the dynamics of this system have been well documented[10]. The pulse sequence shown in Figure 6.7 


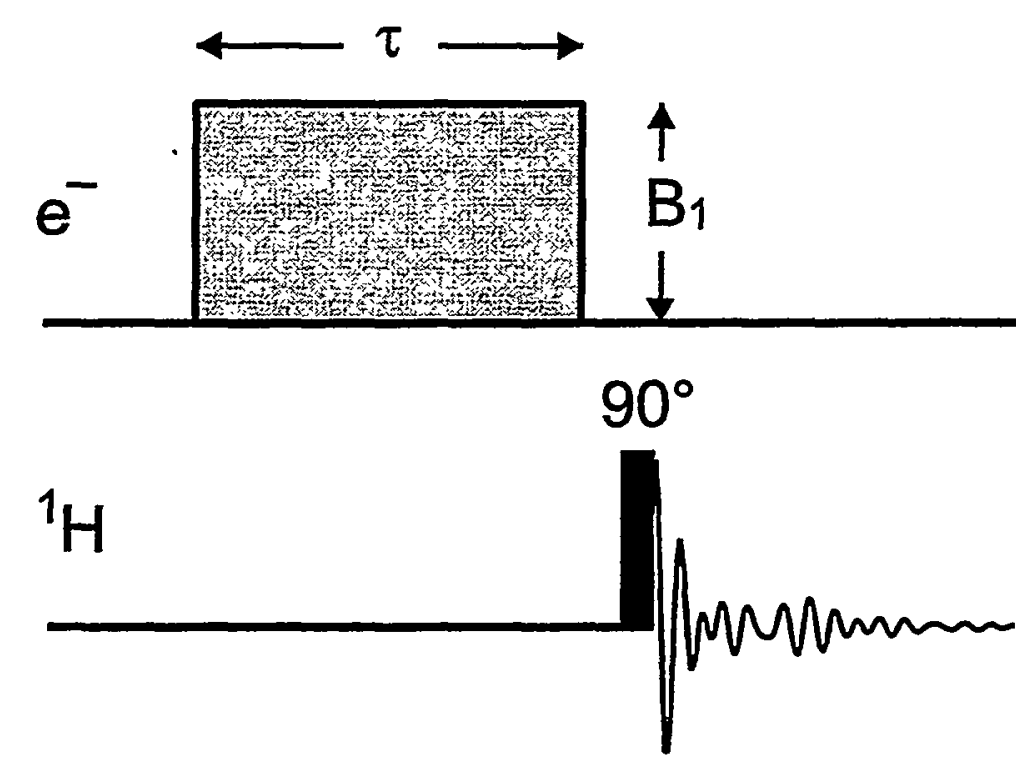

\section{time}

Figure 6.6: Pulse sequence : The grey area is a $75 \mathrm{MHz}$ pulse used to saturate the electrons, while the $90^{\circ}$ pulse is at $120 \mathrm{kHz}$ to excite the protons.

consists of an $r f$ pulse of variable magnetic field amplitude $B_{1}$ and length $\tau$ to saturate the electron spins followed by a $90^{\circ}$ af pulse to interrogate the protons.

The spectra obtained for $\tau=20 \mathrm{~ms}$ and for variable of $B_{1}$ field are shown in Figure 6.8 , where each spectrum is again the result of 400 acquisitions. The increase of the ${ }^{1} \mathrm{H}$ amplitude with $r f$ magnetic field from 0.12 to $0.89 \mathrm{mT}$ is consistent with previous $c w$ experiments[11].

For the $\mathrm{e}^{-1} \mathrm{H}$ DNP system, the expected signal enhancement predicted from Eq. 6.1 is about 800 . The data shown in Figures $6.7-8$ do not represent the ultimate enhancement. As can be seen, the amplitude of the ${ }^{1} \mathrm{H}$ spectra did not saturate with respect to time (Figure 6.7) or $r f B_{1}$ (Figure 6.8). The ranges of the $r f$ pulse time and amplitude are at the 


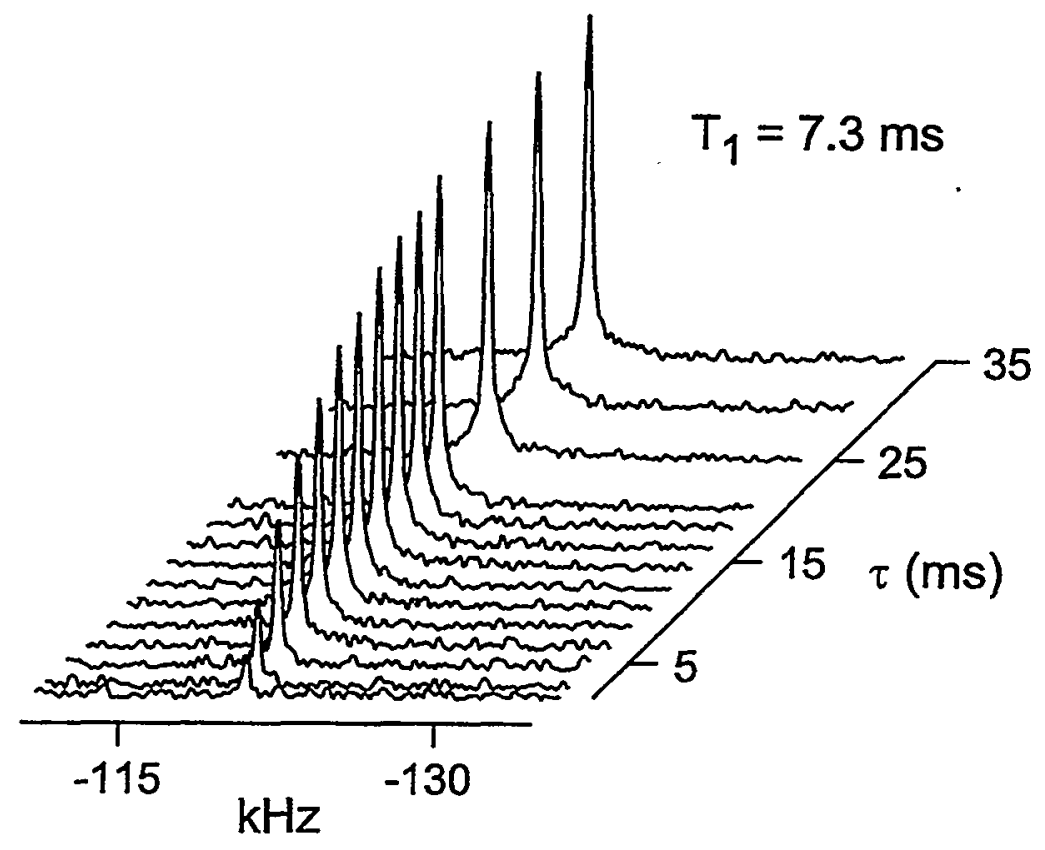

Figure 6.7: $\quad{ }^{1} \mathrm{H}$ spectra observed at $B_{0}=2.7 \mathrm{mT}$ vs $\tau$. Each spectrum was averaged over 400 scans. The inferred $T_{1}$ is $7.3 \mathrm{~ms}$.

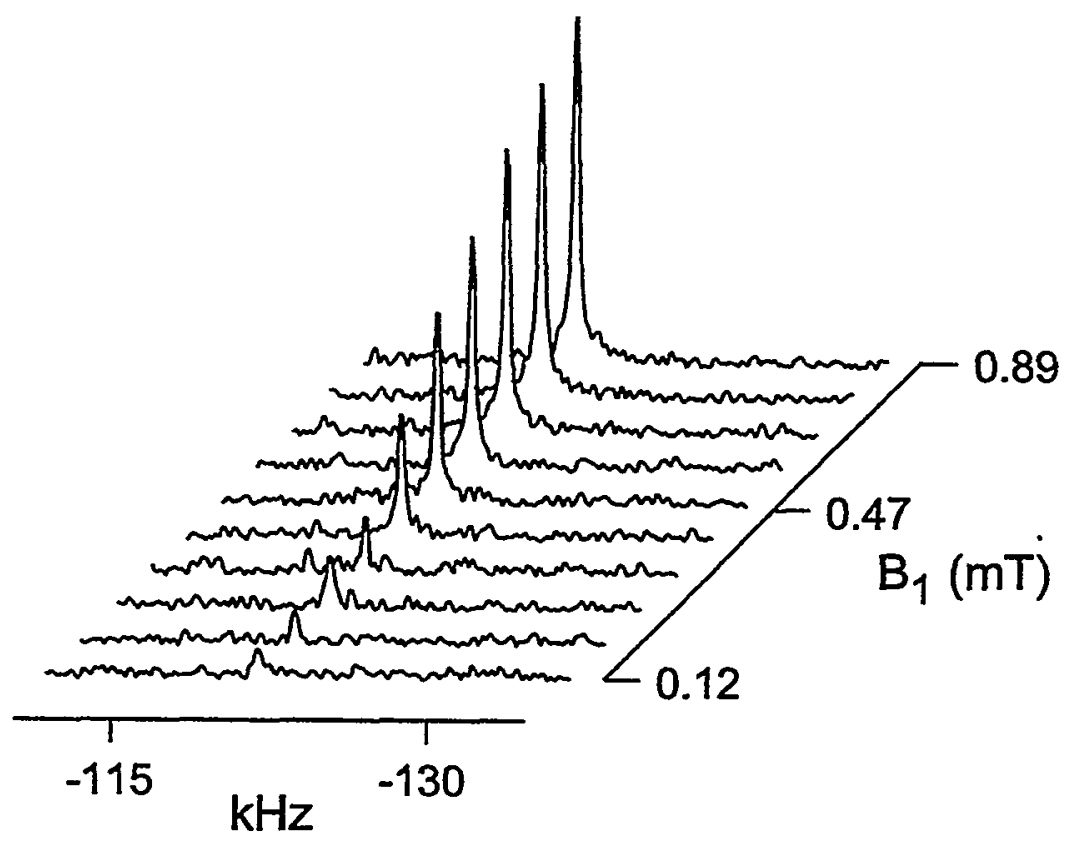

Figure 6.8: $\quad{ }^{1} \mathrm{H}$ spectra observed at $B_{0}=2.7 \mathrm{mT}$ vs magnetic field amplitude $B_{1}$ of $r f$ pulse. Each spectrum was averaged over 400 scans. Note $B_{1}$ scale is logarithmic 

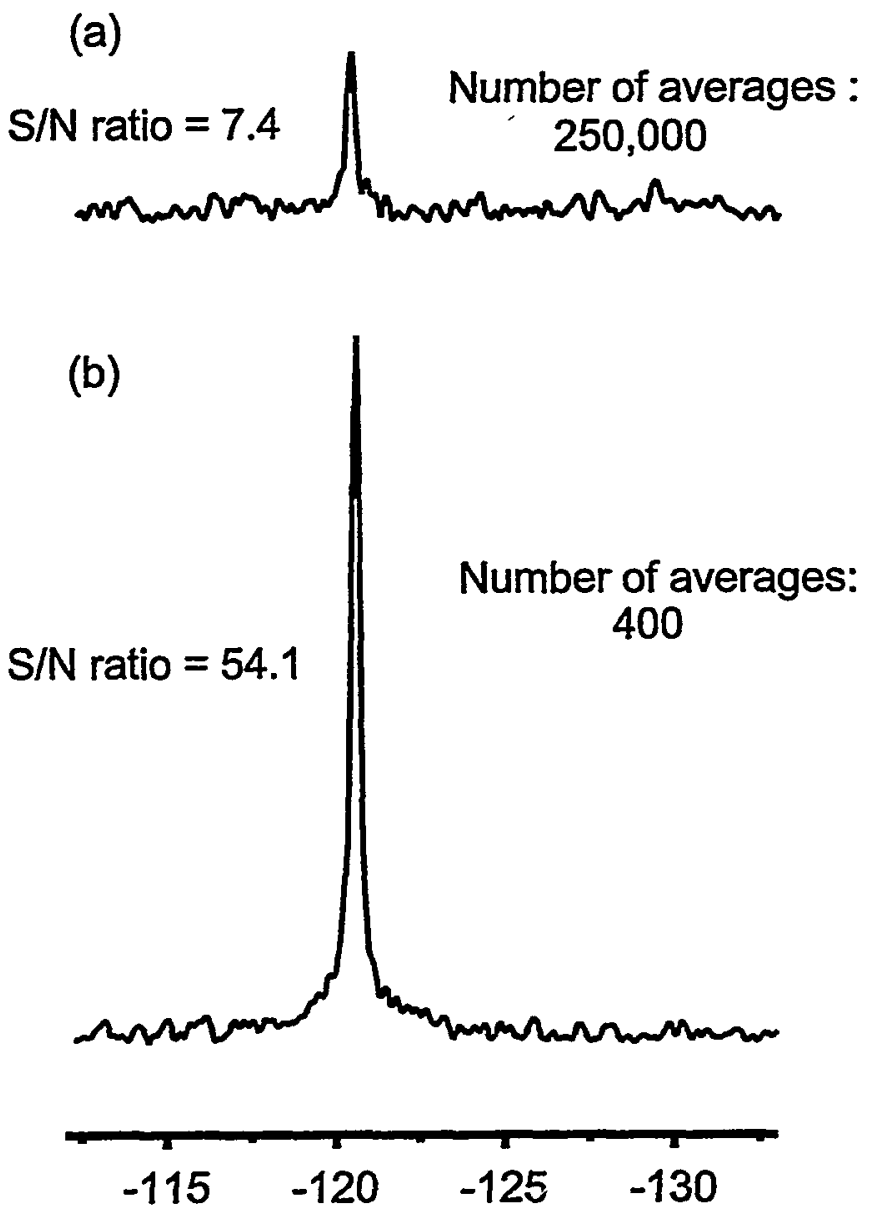

Figure 6.9: Comparison of the ${ }^{1} \mathrm{H}$ spectra at $B_{0}=2.7 \mathrm{mT}$ (a) without and (b) with DNP. The enhancement due to DNP is 183 .

maximum upper limit of the capability of the ENP LPI-10 pulse amplifier; both longer times and higher powers caused the amplifier to shutdown. Nevertheless, even with thelimited power available, the DNP enhancement, shown in Figure 6.9, is about 183.

The af channel is untuned allowing for a broad bandwidth; thus, this allows for the examination of the effect of the static magnetic field on the DNP. In this experiment, the if $75 \mathrm{MHz}$ field is fixed while adjusting the static field $B_{0}$ and the af frequency of the $90^{\circ}$ pulse. The field dependence of the DNP measured this way was unexpectedly broad. For 
example, substantially enhanced proton polarization persisted for proton Larmor frequencies of at least $250 \mathrm{kHz}$, implying that the electron full width bandwidth is at least 150 MHz.

\subsection{SQUID DNP Experiment}

With some experience in performing low-field DNP NMR experiment, we have set up a DNP experiment using a SQUID spectrometer. The schematics of the DNP setup is shown in Figure 6.10. The $r f$ trap and the matching capacitor isolate the af and the $r f$ chan-

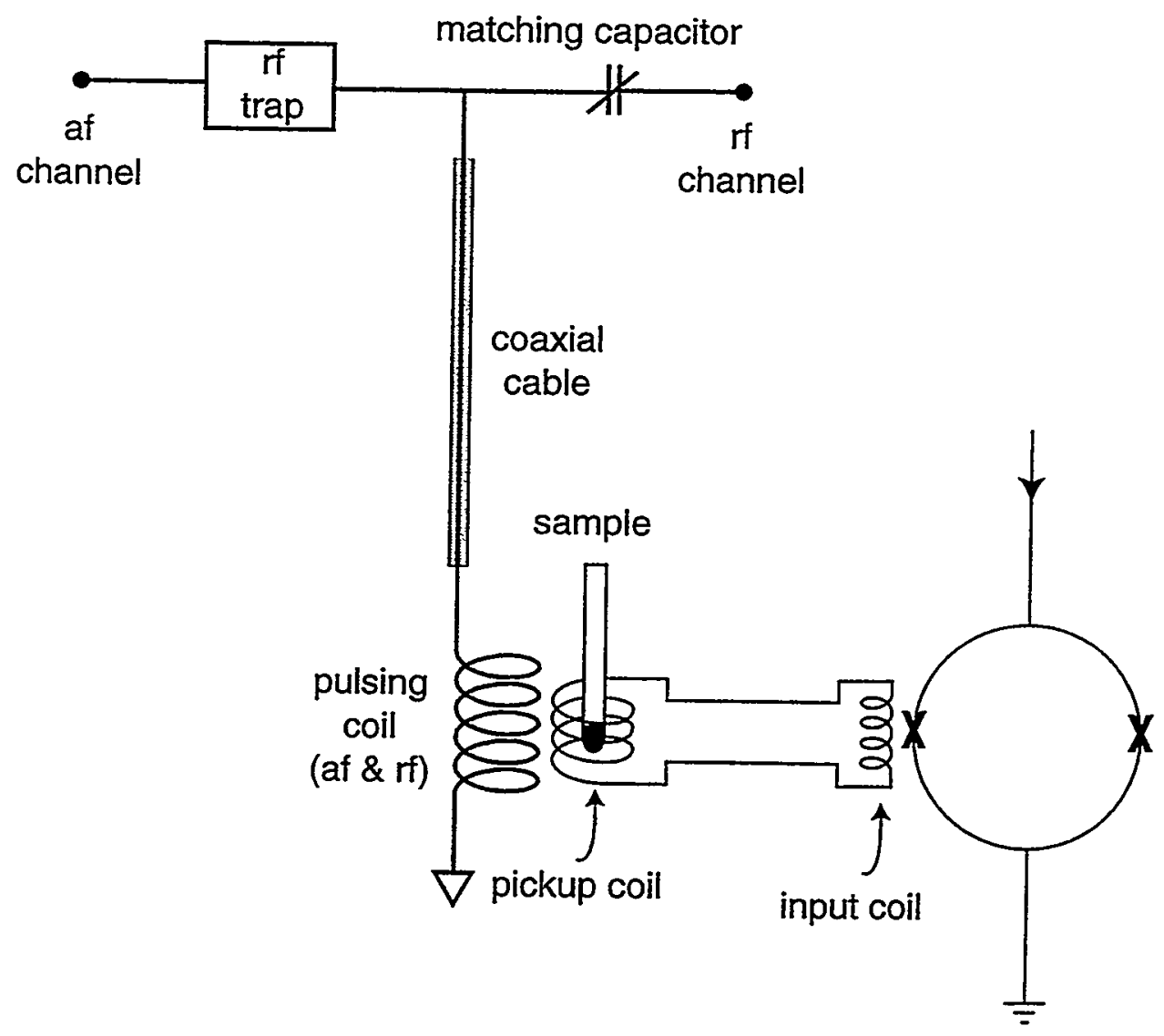

Figure 6.10: The schematics for the the SQUID DNP setup. 
nel during pulsing. The coaxial cable is adjustable to be $\lambda / 2$ at the if frequency for optimum $r f$ pulsing. The sample currently being studied is platinum metal. Other interesting samples include indium phosphate and spin-labled amino acids. 


\section{References}

[1] B. Driehuys, GD. Cates, W. Mabuchi, et al. Phys. Lett. A 184 (1993) 88.

[2] C.R. Bowers, H.W. Long, T. Pietrass, H. Gaede, A. Pines, Chem. Phys. Lett. 205 (1993) 168.

[3] G. Navon, Y-Q. Song, T. Room, S. Appelt, R.E. Taylor, and A. Pines, Science 271 (1996) 1848.

[4] T. Room, S. Appelt, R. Seydoux, E.L. Hahn, A. Pines, Phys. Rev. B 55 (1997) 11604.

[5] H.C. Gaede, Y.-Q. Song, R.E. Taylor, E.J. Munson, et. al., Appl. Mag. Res. 8 (1995) 373.

[6] C.P. Slichter, Priciples of Magnetic Resonance, (Springer-Verlag, Berlin, 1983.)

[7] V. Cross, R. Hester, and J. Waugh, Rev. Sci. Instrum. 47 (1976) 1486.

[8] E. Dormann, G. Sachs, W. Stocklein, B. Bail, and M. Schworer, Appl. Phys. A 30 (1983) 227.

[9] M. Tinkham, R. Weinstein, and A.F. Kip, Phys. Rev. 84 (1951) 848.

[10] R.S. Codrington and N. Bloembergen, J. Chem. Phys. 29 (1958) 600.

[11] A. Overhauser, Phys. Rev. 92 (1953) 411. 


\section{Appendix A: SQUID Electronics}

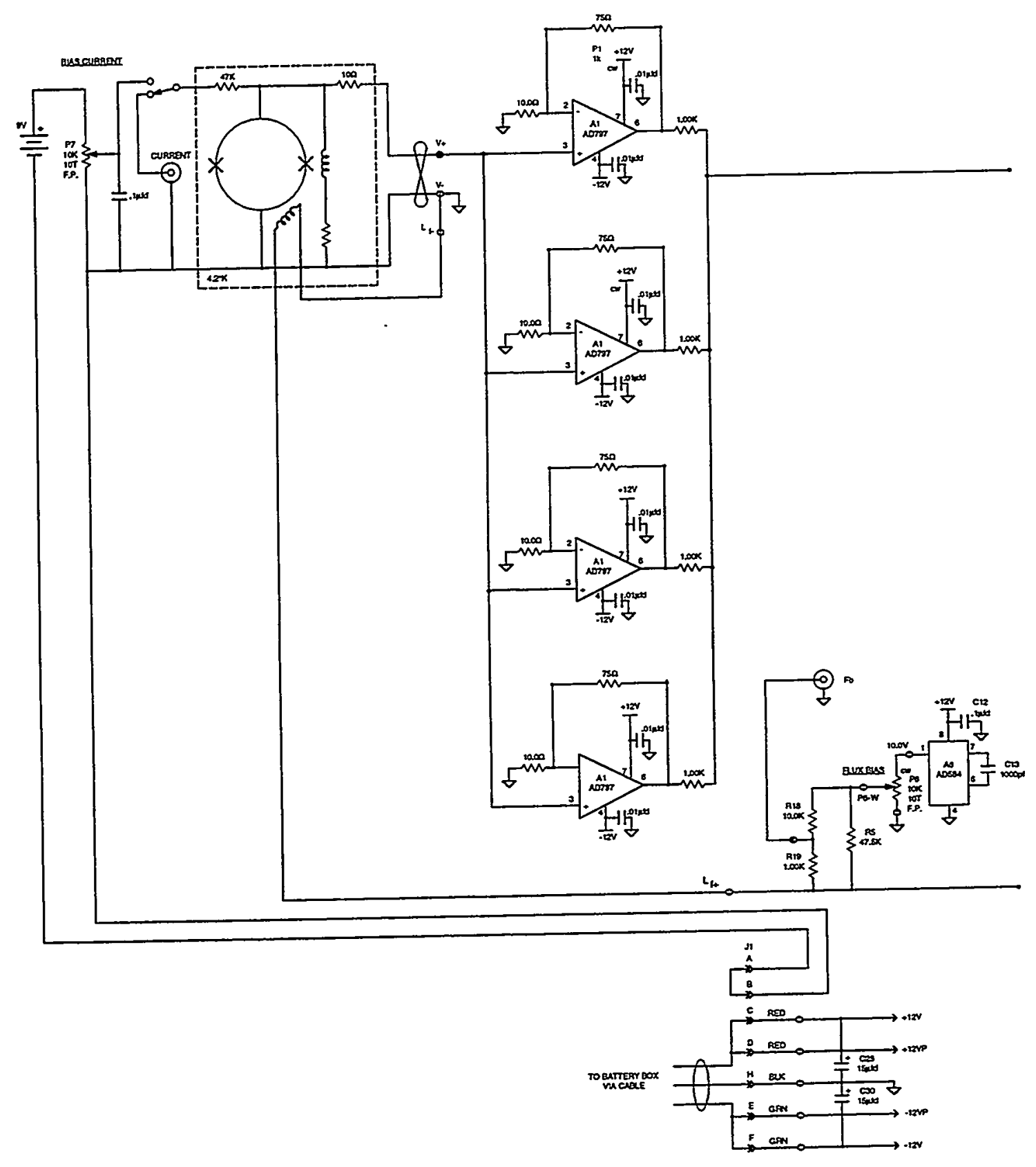

The schematics continues on the next page. 


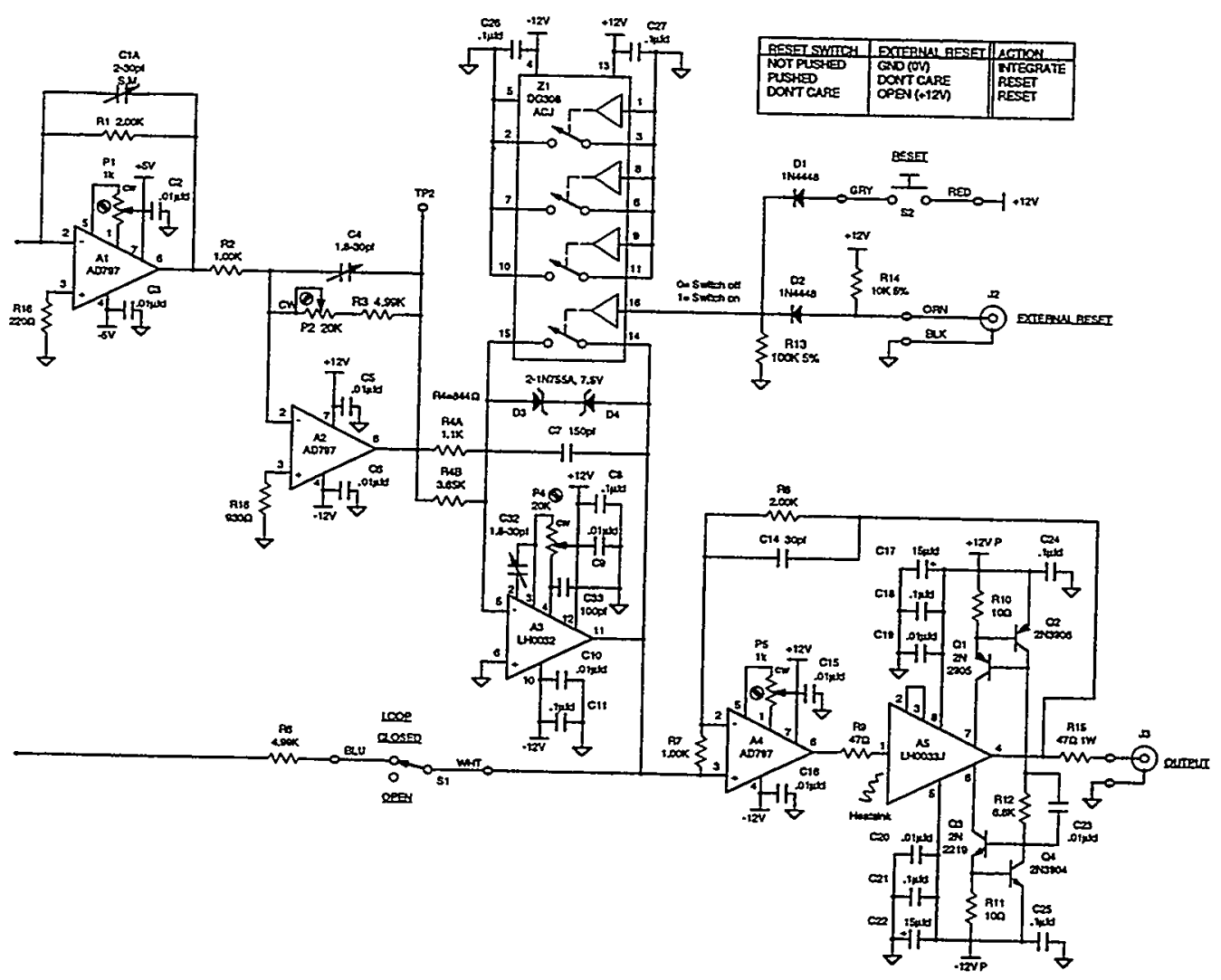

